\title{
Stereoselective Synthesis of Polyhydroxyl Surfactants. Stereochemical Influence on Langmuir Monolayers
}

\author{
Kristina Neimert-Andersson ${ }^{\dagger}$, Eva Blomberg ${ }^{*}$, and Peter Somfai* ${ }^{\dagger} \dagger$ \\ ${ }_{\dagger}^{\dagger}$ KTH Chemistry, Organic Chemistry, ${ }^{\ddagger}$ KTH Chemistry, Surface Chemistry, S-100 44 Stockholm, Sweden. \\ Fax: +46-8-791 2333. E-mail: somfai@kth.se

\section{Supporting Information}

1. Preparation of $\mathbf{1 4}$ from ent-6. $\mathrm{S} 2$

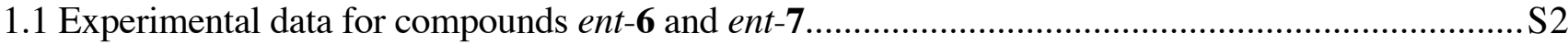

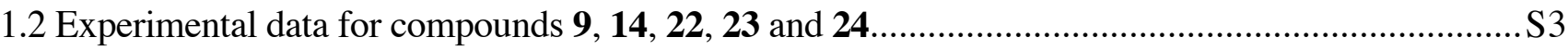

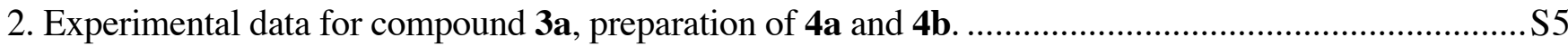

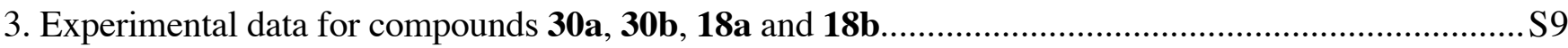

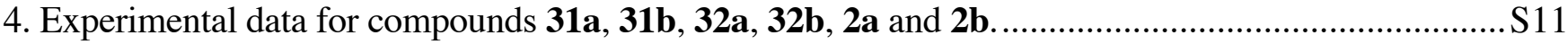

5. Analytical data for compounds ent-21a, ent-21b, ent-1a and ent-1b.........................................S13

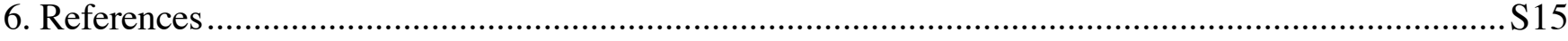

7. NMR spectra for compounds:

1a, b, ent-1a, b, 2a, b, 4a, b, ent-6, 7, ent-7, 8-17, 20a, b, 21a, b, ent-21a, b, 22-24, 27, 30b, 31a, b, 32a, b. 
General. ${ }^{1} \mathrm{H}$ and ${ }^{13} \mathrm{C}$ NMR spectra were recorded in $\mathrm{CDCl}_{3}, \mathrm{CD}_{3} \mathrm{OD}$ or $\mathrm{C}_{6} \mathrm{D}_{6}$ using the residual peak of the corresponding solvent or added TMS $(\delta 0.00)$, as internal standard. Chemical shifts are reported in

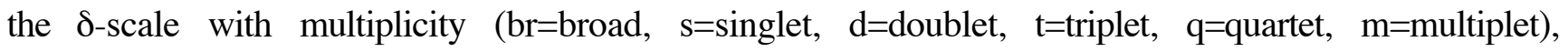
integration and coupling constants $(\mathrm{Hz})$. Optical rotations, $[\alpha]_{\mathrm{D}} \quad$ measured the sodium $\mathrm{D}$ line at ambient temperature. Only the strongest/structurally most important peaks $\left(v, \mathrm{~cm}^{-1}\right)$ are listed in infrared spectra. Analytical thin layer chromatography plates were visualized with UV light and phosphomolybdic acid staining reagent ( $5 \mathrm{w} \%$ solution in $\mathrm{EtOH})$ or $p$-anisaldehyde (solution in EtOH). Air-and moisture sensitive reactions were carried out in flamed-dried, septum-capped flasks under an atmospheric pressure of nitrogen. All liquid reagents were transferred via oven-dried syringes. THF was freshly distilled from sodium-benzophenone ketyl; dichloromethane from $\mathrm{CaH}_{2}$. Surface pressure-area isotherms were recorded on a computerized trough system of the single barrier movement type developed by KSV Instruments, Finland, mounted on an antivibrational table in a laminar flow cabinet. The trough was made of Teflon whereas the barrier was made of Delrin. Millipore filtered water was used as subphase in all experiments. Surface pressure was measured continuously with a Wilhelmy plate. The spreading solvent was $\mathrm{CHCl}_{3}$ with $\mathrm{MeOH}$ added up to $10 \%$ by volume. The concentration of the surfactants in the spreading solutions was $0.5 \mathrm{mM}$, and a volume of $100 \mu \mathrm{L}$ was spread (using a micro syringe). Up to five minutes was allowed for the spreading solvent to evaporate before compression. The compression of the monolayer was carried out at ambient temperature, at a speed of $5.4 \AA^{2}$ molecule ${ }^{-1} \min ^{-1}$ to a molecular area of $100 \AA^{2}$ molecule ${ }^{-1}$ then at a speed of $0.5 \AA^{2}$ molecule ${ }^{-1} \min ^{-1}$. Enantiomerically pure surfactants 1, ent-1 and $\mathbf{2}$ were recrystallized from $\mathrm{H}_{2} \mathrm{O}: \mathrm{MeOH}$ prior to use. The racemate ( $r a c-\mathbf{1}$ ) was prepared by mixing appropriate amounts of $\mathbf{1}$ and ent-1.

\section{Preparation of 14 from ent-6.}

\subsection{Experimental data for compounds ent-6 and ent-7}

\section{(E)-3-((4R,5R)-5-((4-Methoxybenzyloxy)methyl)-2,2-dimethyl-1,3-dioxolan-4-yl)prop-2-en-1-}

ol (ent-6) was prepared as previously reported, ${ }^{1}$ using AD-mix $\beta$ in place of AD-mix $\alpha$. This gave a yield of $84 \%$ and an $e e$ of $>99 \%$ according to $\mathrm{HPLC}$ using chiral column. ${ }^{1} \mathrm{H}$ NMR $\left(400 \mathrm{MHz}, \mathrm{CDCl}_{3}\right) \delta 7.28$ - 
$7.24(\mathrm{~m}, 2 \mathrm{H}), 6.88(\mathrm{~d}, J=8.6 \mathrm{~Hz}, 2 \mathrm{H}), 5.93(\mathrm{td}, J=15.5,5.1 \mathrm{~Hz}), 5.72(\mathrm{tdd}, J=15.5,7.6,1.5 \mathrm{~Hz}, 1 \mathrm{H}), 4.52(\mathrm{~s}$, $2 \mathrm{H}), 4.24(\mathrm{t}, J=7.6 \mathrm{~Hz}, 1 \mathrm{H}), 4.17-4.14(\mathrm{~m}, 2 \mathrm{H}), 3.92-3.88(\mathrm{~m}, 1 \mathrm{H}), 3.81(\mathrm{~s}, 3 \mathrm{H}), 3.60-3.52(\mathrm{~m}, 2 \mathrm{H}), 1.60$ (br s, 1H), $1.43(\mathrm{~s}, 6 \mathrm{H}) ;{ }^{13} \mathrm{C}$ NMR (100 MHz, $\left.\mathrm{CDCl}_{3}\right) \delta 159.7,134.5,130.5,129.8,128.2,114.1,109.8$, 80.6, 79.0, 77.7, 73.7, 69.5, 63.1, 55.6, 27.5; IR (neat) 3432 (br) $\mathrm{cm}^{-1} ;[\alpha]_{\mathrm{D}}+14.4\left(c 1.00, \mathrm{CH}_{2} \mathrm{Cl}_{2}\right)$; HRMS $(\mathrm{FAB}+)$ calcd for $\mathrm{C}_{17} \mathrm{H}_{24} \mathrm{O}_{5}(\mathrm{M}): 308.1624$, found: 308.1622 .

(E)-3-((4R,5R)-5-((4-Methoxybenzyloxy)methyl)-2,2-dimethyl-1,3-dioxolan-4-yl)allyl

methoxybenzoate (ent-7) was prepared from ent-6 as described for 7. ${ }^{1} \mathrm{H}$ NMR $\left(500 \mathrm{MHz}, \mathrm{CDCl}_{3}\right) \delta$ $8.02(\mathrm{~d}, J=8.8 \mathrm{~Hz}, 2 \mathrm{H}), 7.28$ (d, J=8.4 Hz, 2H), 6.94 (d, J=8.8 Hz, 2H), 6.89 (d, J=8.4 Hz, 2H), 6.01 (td, $J=15.5,5.7 \mathrm{~Hz}, 1 \mathrm{H}), 5.87$ (dd, J=15.5, 7.2 Hz, 1H), 4.81 (d, J=5.7 Hz, 2H), 4.56 (AB-d, J=11.8 Hz, 1H), 4.54 (AB-d, J=11.8 Hz, 1H), 4.31 (t, J=7.2 Hz, 1H), 3.96-3.92 (m, 1H), 3.89 (s, 3H), 3.81 (s, 3H), 3.63$3.57(\mathrm{~m}, 2 \mathrm{H}), 1.47(\mathrm{~s}, 6 \mathrm{H}) ;{ }^{13} \mathrm{C}$ NMR $\left(125 \mathrm{MHz}, \mathrm{CDCl}_{3}\right) \delta$ 166.3, 163.9, 159.6, 132.1, 131.1, 130.4, 129.7, $129.1,122.8,114.2,114.1,109.9,80.4,78.8,73.7,69.5,64.5,55.9,55.7,27.4,27.4$; IR (neat) 2986, 1713, $1607 \mathrm{~cm}^{-1} ;[\alpha]_{\mathrm{D}}+15.7\left(c\right.$ 1.04, $\left.\mathrm{CH}_{2} \mathrm{Cl}_{2}\right)$; HRMS (FAB+) calcd for $\mathrm{C}_{25} \mathrm{H}_{31} \mathrm{O}_{7}(\mathrm{M}+\mathrm{H})$ : 443.2070, found: 443.2077 .

\subsection{Experimental data for compounds 9, 14, 22, 23 and 24}

Scheme S1. Preparation of 14 from 9.<smiles>[R9]C[C@]1(CO)OC[C@@H]([C@H](O)[C@H](O)CO)[C@H]1C(C)(C)C</smiles>

$9 \mathrm{R}=\mathrm{PMB} \mathrm{R}^{1}=\mathrm{PMBz}$

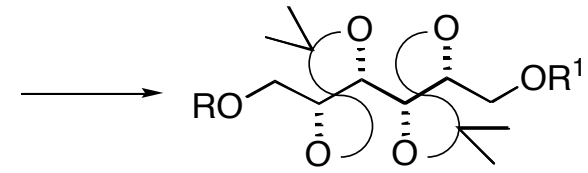

$22 \mathrm{R}=\mathrm{PMB} \mathrm{R}^{1}=\mathrm{PMBz}$

$23 \mathrm{R}=P M B \mathrm{R}^{1}=\mathrm{H}$

$24 R=P M B R^{1}=T s$

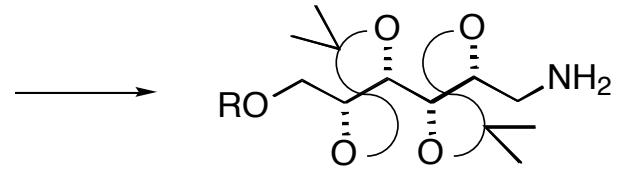

$14 \mathrm{R}=\mathrm{PMB}$

2,3-O-Isopropylidene-1-(4-methoxybenzyl)-D-iditol-6-(4-methoxybenzoate) (9) was prepared as describes for $\mathbf{8}$ and obtained in $82 \%$ yield and a diastereomeric ratio of 33:1 after flash chromatography 
(EtOAc:pentane 0:1 $\rightarrow 1: 1) .{ }^{1} \mathrm{H}$ NMR $\left(400 \mathrm{MHz}, \mathrm{CDCl}_{3}\right) \delta 7.97(\mathrm{~d}, J=8.2 \mathrm{~Hz}, 2 \mathrm{H}), 7.21(\mathrm{~d}, J=8,5 \mathrm{~Hz}, 2 \mathrm{H})$, $6.90(\mathrm{~d}, J=8.2 \mathrm{~Hz}, 2 \mathrm{H}), 6.83(\mathrm{~d}, J=8.5 \mathrm{~Hz}, 2 \mathrm{H}), 4.48(\mathrm{~s}, 2 \mathrm{H}), 4.41$ (d, J=5.7 Hz, 2H), 4.30 (td, J=8.1, 5.2 $\mathrm{Hz}, 1 \mathrm{H}), 4.11-4.07(\mathrm{~m}, 1 \mathrm{H}), 4.00(\mathrm{dd}, J=8.1,2.7 \mathrm{~Hz}, 2 \mathrm{H}), 3.85$ (s, 3H), 3.77 (s, 3H), 3.68 (td, J=9.0, 2.7 Hz, 1H), 3.64 (dd, J=10.2, $5.2 \mathrm{~Hz}, 1 \mathrm{H}), 3.55$ (dd, J=10.2, $5.2 \mathrm{~Hz}, 1 \mathrm{H}), 2.97$ (d, J=3.4 Hz, 1H), 2.70 (d, $J=9.0 \mathrm{~Hz}, 1 \mathrm{H}), 1.43(\mathrm{~s}, 3 \mathrm{H}), 1.41(\mathrm{~s}, 3 \mathrm{H}) ;{ }^{13} \mathrm{C} \mathrm{NMR}\left(125 \mathrm{MHz}, \mathrm{CDCl}_{3}\right) \delta 166.3,163.6,159.3,131.8$, $129.7,129.4,122.2,113.9,113.7,109.9,80.8,75.8,73.3,71.3,69.8,69.1,65.3,55.4,55.2,27.1,26.8 ;$ IR (neat): 3474 (br), $1607 \mathrm{~cm}^{-1} ;[\alpha]_{\mathrm{D}}+8.1\left(c\right.$ 1.02, $\left.\mathrm{CH}_{2} \mathrm{Cl}_{2}\right)$; HRMS (FAB+) calcd for $\mathrm{C}_{25} \mathrm{H}_{33} \mathrm{O}_{9}(\mathrm{M}+\mathrm{H})$ : 477.2125, found: 477.2126 .

2,3:4,5-Di- $O$-isopropylidene-1-(4-methoxybenxyl)-D-iditol-6-(4-methoxybenzoate) (22) was prepared from 9 as described for $\mathbf{1 0}$ and obtained in 53\% yield. ${ }^{1} \mathrm{H}$ NMR $\left(400 \mathrm{MHz}, \mathrm{C}_{6} \mathrm{D}_{6}\right) \delta 7.93(\mathrm{~d}$, $J=8.9 \mathrm{~Hz}, 2 \mathrm{H}), 6.94(\mathrm{~d}, J=8.5 \mathrm{~Hz}, 2 \mathrm{H}), 6.56(\mathrm{~d}, J=8.5 \mathrm{~Hz}), 6.38(\mathrm{~d}, J=8.9 \mathrm{~Hz}, 2 \mathrm{H}), 4.46-4.41(\mathrm{~m}, 1 \mathrm{H})$, 4.37-4.33 (m, 1H), 4.31 (dd, J=11.8, 3.9 Hz, 1H), 4.22 (dd, J=11.8, 5.5 Hz, 1H), 4.09 (s, 2H), 3.88-3.84 (m, 2H), 3.39 (dd, J=10.1, 4.6 Hz, 1H), 3.32 (dd, J=10.1 Hz, $5.3 \mathrm{~Hz}, 1 \mathrm{H}), 3.09$ (s, 3H), 2.93 (s, 3H), 1.27 (s, 3H), 1.25 (s, 6H), 1.20 (s, 3H); ${ }^{13} \mathrm{C}$ NMR (100 MHz, $\left.\mathrm{C}_{6} \mathrm{D}_{6}\right) \delta$ 166.2, 164.1, 160.1, 132.4, 130.9, 129.7, $123.2,114.4,114.3,110.3,110.0,78.0,77.5,76.8,76.2,73.6,70.7,64.6,55.1,55.0,27.84,27.83,27.22$, 27.19; IR (neat): 2987, 1713, $1607 \mathrm{~cm}^{-1} ;[\alpha]_{\mathrm{D}}+15.8\left(c 1.17, \mathrm{CHCl}_{3}\right)$; HRMS (FAB+) calcd for $\mathrm{C}_{28} \mathrm{H}_{36} \mathrm{O}_{9}$ (M): 516.2359, found: 516.2360.

2,3:4,5-Di- $O$-isopropylidene-1-(4-methoxybenxyl)-D-iditol (23) was prepared from 22 as described for 11 and obtained in $90 \%$ yield. ${ }^{1} \mathrm{H}$ NMR: (400 MHz, $\left.\mathrm{CDCl}_{3}\right) \delta 7.31(\mathrm{~d}, J=8.4 \mathrm{~Hz}, 2 \mathrm{H}), 6.93$ (d, $J=8.4 \mathrm{~Hz}, 2 \mathrm{H}) 4.58$ (AB-d, $J=11.7 \mathrm{~Hz}, 1 \mathrm{H}), 4.55$ (AB-d, $J=11.7 \mathrm{~Hz}, 1 \mathrm{H}), 4.32-4.28$ (m, 1H), 4.22-4.18 (m, 1H), $4.02(\mathrm{dd}, J=8.3,3.2 \mathrm{~Hz}, 1 \mathrm{H}), 3.92(\mathrm{dd}, J=8.3,3.3 \mathrm{~Hz}, 1 \mathrm{H}), 3.86(\mathrm{~s}, 3 \mathrm{H}), 3.84-3.81$ (m, 1H), 3.69$60(\mathrm{~m}, 3 \mathrm{H}), 2.04(\mathrm{dd}, J=7.8,4.8 \mathrm{~Hz}, 1 \mathrm{H}), 1.47(\mathrm{~s}, 9 \mathrm{H}), 1.46(\mathrm{~s}, 3 \mathrm{H}) ;{ }^{13} \mathrm{C} \mathrm{NMR}\left(125 \mathrm{MHz}, \mathrm{CDCl}_{3}\right)$ : $\delta 159.7,130.3,129.8,114.2,110.1,109.9,77.8,77.72$ 76.6, 76.5, 73.6, 70.7, 62.0, 55.7, 27.63, 27.60, 27.2, 27.1; IR (neat): 3438 (br), $1613 \mathrm{~cm}^{-1} ;[\alpha]_{\mathrm{D}}+19.3$ (c 1.00, $\mathrm{CHCl}_{3}$ ); HRMS (FAB+) calcd for $\mathrm{C}_{20} \mathrm{H}_{30} \mathrm{O}_{7}$ (M): 382.1992, found: 382.1990. 
was prepared form $\mathbf{2 3}$ as described for $\mathbf{1 2}$ and obtained in $99 \%$ yield. ${ }^{1} \mathrm{H}$ NMR (500 MHz, $\mathrm{CDCl}_{3}$ ) $\delta 7.80$ (d, $J=8.2 \mathrm{~Hz}, 2 \mathrm{H}), 7.34$ (d, J=8.2 Hz, 2H), 7.27 (d, $J=8.7 \mathrm{~Hz}, 2 \mathrm{H}), 6.91$ (d, $J=8.7 \mathrm{~Hz}, 2 \mathrm{H}), 4.54$ (AB-d, $J=11.7 \mathrm{~Hz}, 1 \mathrm{H}) 4.52(\mathrm{AB}-\mathrm{d}, J=11.7 \mathrm{~Hz}, 1 \mathrm{H}), 4.25-4.15(\mathrm{~m}, 3 \mathrm{H}), 4.10(\mathrm{dd}, J=10.8,4.8 \mathrm{~Hz}, 1 \mathrm{H}), 3.92(\mathrm{dd}$, $J=8.1,3.2 \mathrm{~Hz}, 1 \mathrm{H}), 3.86(\mathrm{dd}, J=8.1,3.2 \mathrm{~Hz}, 1 \mathrm{H}), 3.83(\mathrm{~s}, 3 \mathrm{H}), 3.60(\mathrm{dd}, J=10.2,5.3 \mathrm{~Hz}, 1 \mathrm{H}), 3.55$ (dd, $J=10.2,4.9 \mathrm{~Hz}, 1 \mathrm{H}), 2.46$ (s, 3H), 1.42 (s, 3H), 1.40 (s, 3H), 1.37 (s, 3H), 1.35 (s, 3H); ${ }^{13} \mathrm{C}$ NMR $(125$ $\left.\mathrm{MHz}, \mathrm{CDCl}_{3}\right) \delta 159.2,144.9,132.6,129.8,129.4,128.0,113.8,110.1,109.7,77.1,76.2,75.9,74.5,73.1$, 69.9, 68.7, 55.2, 27.1, 26.8, 26.6, 26.5, 21.6; one signal is not visible; IR (neat): 1613, 1368, $1177 \mathrm{~cm}^{-1}$; $[\alpha]_{\mathrm{D}}+19.2\left(c 1.00, \mathrm{CHCl}_{3}\right)$; HRMS (FAB+) calcd for $\mathrm{C}_{27} \mathrm{H}_{36} \mathrm{O}_{9} \mathrm{~S}(\mathrm{M}): 536.2080$, found: 536.2084.

1-Amino-1-deoxy-2,3:4,5-di- $O$-isopropylidene-D-iditol-6-(4-methoxybenzoate)

(14) was prepared form $\mathbf{2 4}$ as described for $\mathbf{1 3}$ and obtained in $77 \%$ yield. ${ }^{1} \mathrm{H}$ NMR (400 MHz, $\left.\mathrm{CDCl}_{3}\right) \delta 7.20(\mathrm{~d}$, $J=8.7 \mathrm{~Hz}, 2 \mathrm{H}), 6.82(\mathrm{~d}, J=8.7 \mathrm{~Hz}, 2 \mathrm{H}), 4.47$ (AB-d, $J=11.7 \mathrm{~Hz}, 1 \mathrm{H}), 4.45$ (AB-d, $J=11.7 \mathrm{~Hz}, 1 \mathrm{H}), 4.20$ (td, $J=8.2,4.9 \mathrm{~Hz}, 1 \mathrm{H}), 4.03-3.99(\mathrm{~m}, 1 \mathrm{H}), 3.80(\mathrm{dd}, J=8.2,3.1 \mathrm{~Hz}), 3.74(\mathrm{~s}, 1 \mathrm{H}), 3.72(\mathrm{dd}, J=8.3,3.1 \mathrm{~Hz}, 1 \mathrm{H})$, 3.54 (AB-dd, J=10.3, $5.2 \mathrm{~Hz}, 1 \mathrm{H}$ ), 3.51 (AB-dd. $J=10.3,4.6 \mathrm{~Hz}, 1 \mathrm{H}$ ), 2.87 (br d, $J=13.2 \mathrm{~Hz}, 1 \mathrm{H}$ ), 2.72 (dd, $J=13.2,6.5 \mathrm{~Hz}, 1 \mathrm{H}), 1.98$ (br s, 2H), 1.37-1.36 (m, 9H), 1.34 (s, 3H); ${ }^{13} \mathrm{C}$ NMR (100 MHz, $\mathrm{CDCl}_{3}$ ) $\delta$ 159.2, 129.9, 129.4, 113.8, 109.6, 109.2, 78.7, 77.4, 77.1, 76.1, 73.2, 69.9, 55.3, 43.7, 27.3, 27.2, 26.70, 26.69; IR (neat): 2987, $1514 \mathrm{~cm}^{-1} ;[\alpha]_{\mathrm{D}}+23.4\left(c\right.$ 1.00, $\mathrm{CHCl}_{3}$ ); HRMS (FAB+) calcd for $\mathrm{C}_{20} \mathrm{H}_{32} \mathrm{NO}_{6}$ $(\mathrm{M}+\mathrm{H}): 382.2230$, found: 382.2227 .

\section{Experimental data for compound 3a, preparation of $4 \mathrm{a}$ and $\mathbf{4 b}$.}

Experimental procedure for the preparation of dimethyl 2-butyl malonate (3a). ${ }^{2} \mathrm{NaH}(584 \mathrm{mg}$, $14.6 \mathrm{mmol}, 60 \mathrm{w} \%$ dispersion in oil) was washed twice with pentane, dried under vacuum and resuspended in THF/DMF $(3: 1,40 \mathrm{~mL})$ at $0{ }^{\circ} \mathrm{C}$. Dimethyl malonate $(4.2 \mathrm{~mL}, 36.5 \mathrm{mmol})$ was added dropwise and the 
clear solution was stirred for $15 \mathrm{~min}$ at $\mathrm{rt}$, at which white crystals were formed. $n$-Butyl bromide $(0.78 \mathrm{~mL}$, $7.3 \mathrm{mmol}$ ) was added and the mixture heated to reflux. After refluxing overnight the reaction mixture was allowed to cool, the THF was removed under vacuum and the residue poured into $\mathrm{H}_{2} \mathrm{O}(20 \mathrm{~mL})$ and extracted three times with pentane $(15 \mathrm{~mL})$ and once with $\mathrm{Et}_{2} \mathrm{O}$ /pentane $(1: 1,20 \mathrm{~mL})$. The organic layers were combined, dried $\left(\mathrm{MgSO}_{4}\right)$ and the solvents were evaporated to give a yellow oil. Excess dimethyl malonate and dialkylated product was removed with repeated fractional distillation $\left(2\right.$ torr, $\left.45{ }^{\circ} \mathrm{C}\right)$ to give 75\% yield of 3a (1.0 g, $5.4 \mathrm{mmol}) .{ }^{1} \mathrm{H}$ NMR (400 MHz, $\left.\mathrm{CDCl}_{3}\right) \delta 3.74(\mathrm{~s}, 6 \mathrm{H}), 3.36(\mathrm{t}, J=7.6 \mathrm{~Hz}, 1 \mathrm{H})$, $1.90(\mathrm{q}, J=7.6 \mathrm{~Hz}, 2 \mathrm{H}), 1.39-1.25(\mathrm{~m}, 4 \mathrm{H}), 0.90(\mathrm{t}, J=7.0 \mathrm{~Hz}, 3 \mathrm{H}) ;{ }^{13} \mathrm{C} \mathrm{NMR}\left(100 \mathrm{MHz}, \mathrm{CDCl}_{3}\right) \delta 170.1$, $52.5,51.7,29.5,28.6,22.3,13.8$.

Scheme S2. Preparation of 4a from 25.

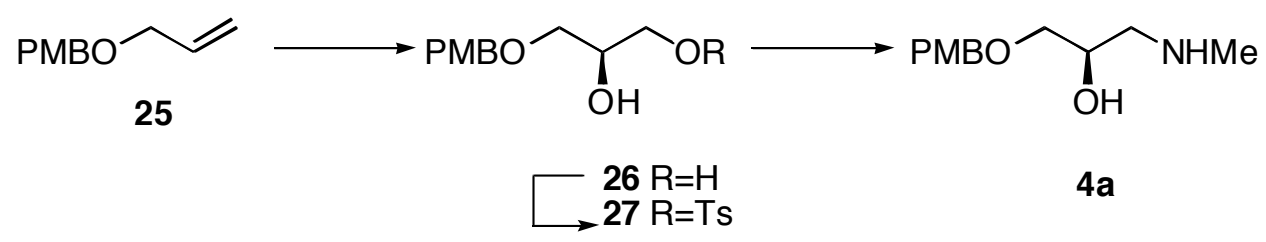

(2R)-3-(4-Methoxybenzyloxy)-1,2-propanediol (26). ${ }^{3}$ Allyl alcohol $\mathbf{2 5}^{4}$ (1.0 g, $\left.5.6 \mathrm{mmol}\right)$ was added to a suspension of $\mathrm{AD}$-mix $\alpha(9.3 \mathrm{~g})$ and $\mathrm{K}_{2} \mathrm{OsO}_{4} \cdot 2 \mathrm{H}_{2} \mathrm{O}(14.4 \mathrm{mg}, 0.039 \mathrm{mmol})$ in $t$ - $\mathrm{BuOH}: \mathrm{H}_{2} \mathrm{O}$ $(1: 1,20 \mathrm{~mL})$. The mixture was stirred at $0{ }^{\circ} \mathrm{C}$ for $45 \mathrm{~h} . \mathrm{Na}_{2} \mathrm{SO}_{3}(7.5 \mathrm{~g})$ was added and the reaction mixture stirred for two more hours at room temperature, then diluted with EtOAc $(40 \mathrm{~mL})$ and $\mathrm{H}_{2} \mathrm{O}(20 \mathrm{~mL})$. The phases were separated and the water phase extracted with EtOAc $(2 \times 30 \mathrm{~mL})$. After drying $\left(\mathrm{MgSO}_{4}\right)$ and evaporation of the solvent $1.1 \mathrm{~g}$ of a crude oil remained. Purification by flash chromatography on silica gel (EtOAc:MeOH 25:1) gave 68\% of $\mathbf{2 6}$ as a white solid (797 mg, $3.8 \mathrm{mmol}$ ). ee: $60 \%$ according to NMR using $\mathrm{Eu}(\mathrm{hfc})_{3}$ as chiral shift reagent; ${ }^{1} \mathrm{H} \mathrm{NMR}\left(400 \mathrm{MHz}, \mathrm{CDCl}_{3}\right) \delta 7.25$ (d, J=8.7 Hz, 2H), 6.89 (d, $J=8.7 \mathrm{~Hz}, 2 \mathrm{H}), 4.48(\mathrm{~s}, 2 \mathrm{H}), 3.90-3.84(\mathrm{~m}, 1 \mathrm{H}), 3.81(\mathrm{~s}, 3 \mathrm{H}), 3.73-3.60(\mathrm{~m}, 2 \mathrm{H}), 3.57-3.49$ (m, $2 \mathrm{H}), 2.57$ $(\mathrm{d}, J=3.9 \mathrm{~Hz}, 1 \mathrm{H}), 2.08$ (br s, 1H); ${ }^{13} \mathrm{C} \mathrm{NMR}\left(100 \mathrm{MHz}, \mathrm{CDCl}_{3}\right) \delta$ 159.8, 130.2, 129.9, 114.3, 73.7, 71.9, $71.0,64.5,55.7$. 
General procdure for tosylation of 29 and 31 employing dibutyl tin oxide as catalyst. (2S)-3(4-Methoxybenzyloxy)-2-hydroxypropyl 4-methylbenzenesulfonat (27). Diol 26 (420 mg, 2.0 mmol) was stirred together with $\mathrm{Bu}_{2} \mathrm{SnO}(9.9 \mathrm{mg}, 0.04 \mathrm{mmol}), p$-TsCl $(379 \mathrm{mg}, 1.42 \mathrm{mmol})$ and $\mathrm{Et}_{3} \mathrm{~N}$ ( $279 \mu \mathrm{L}, 1.42 \mathrm{mmol}$ ) in dry $\mathrm{CH}_{2} \mathrm{Cl}_{2}$ for $4 \mathrm{~h}$. The solvent was evaporated and the residue was suspended in EtOAc:pentane (1:1). The precipitate $\left(\mathrm{Et}_{3} \mathrm{NHCl}\right)$ was filtered off, the filtrate was taken care of and the solvent was evaporated. Purification by flash chromatography on silica gel (EtOAc:pentane 1:1) furnished 27 in $88 \%$ yield as a colorless oil $(640 \mathrm{mg}, 1.76 \mathrm{mmol}) .{ }^{1} \mathrm{H} \mathrm{NMR}\left(400 \mathrm{MHz}, \mathrm{CDCl}_{3}\right) \delta 7.79(\mathrm{~d}, J=8.3 \mathrm{~Hz}$, 2H), 7.34 (d, J=8.4 Hz, 2H), 7.19 (d, J=8.4 Hz, 2H), 6.87 (d, J=8.3 Hz, 2H), 4.43 (s, 2H), 4.13-3.96 (m, $3 \mathrm{H}), 3.81(\mathrm{~s}, 3 \mathrm{H}), 3.52-3.44(\mathrm{~m}, 2 \mathrm{H}), 2.44(\mathrm{~s}, 3 \mathrm{H}) ;{ }^{13} \mathrm{C} \mathrm{NMR}\left(100 \mathrm{MHz}, \mathrm{CDCl}_{3}\right) \delta 159.8,145.5,133.0$, 130.4, 130.0, 129.9, 128.4, 114.3, 73.6, 71.0, 70.1, 68.8, 55.7, 22.1; IR (neat): 3478, 1357, $1175 \mathrm{~cm}^{-1}$; HRMS (FAB+) calcd for $\mathrm{C}_{18} \mathrm{H}_{22} \mathrm{O}_{6} \mathrm{~S}(\mathrm{M}): 366.1137$, found: 366.1143 .

(2R)-1-(Methylamine)-3-(4-methoxybenzyloxy)-propan-2-ol (4a). Tosylate 27 (480 mg, 1.31 mmol), methylamine $(5 \mathrm{~mL}, 40 \mathrm{w} \%$ in water $)$ and $\mathrm{THF}(10 \mathrm{~mL})$ were heated in a sealed tube at $75^{\circ} \mathrm{C}$ for 3h. The reaction mixture was allowed to cool to room temperature, and then THF was evaporated under reduced pressure. The residue was redissolved in $\mathrm{CH}_{2} \mathrm{Cl}_{2}(10 \mathrm{~mL})$ and extracted with $\mathrm{NaOH}(2 \mathrm{M}, 2 \mathrm{~mL})$. The water phase was extracted twice with $\mathrm{CH}_{2} \mathrm{Cl}_{2}(2 \mathrm{~mL})$ and the organic phases dried $\left(\mathrm{MgSO}_{4}\right)$. Evaporation of the solvent gave $\mathbf{4 a}$ in $97 \%$ yield $(285 \mathrm{mg}, 1.27 \mathrm{mmol})$. The product was used without further purification. ${ }^{1} \mathrm{H} \mathrm{NMR}\left(400 \mathrm{MHz}, \mathrm{CDCl}_{3}\right) \delta 7.25(\mathrm{~d}, J=8.5,2 \mathrm{H}), 6.88(\mathrm{~d}, J=8.5,2 \mathrm{H}), 4.48(\mathrm{~s}, 2 \mathrm{H})$, 3.92-3.85 (m, 1H), 3.80 (s, 3H), 3.48 (AB-dd, J=9.7, 4.1 Hz, 1H), 3.43 (AB-d, J=9.7, 6.3 Hz, 1H), 2.69-

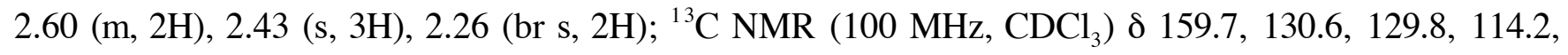
73.5, 73.1, 69.0, 55.7, 54.7, 36.7; IR (neat): 3313 (br), $2840 \mathrm{~cm}^{-1}$; HRMS (FAB+) calcd for $\mathrm{C}_{12} \mathrm{H}_{20} \mathrm{NO}_{3}$ $(\mathrm{M}+\mathrm{H}): 226.1443$, found: 226.1447 . 
Scheme S3. Preparation of $\mathbf{4 b}$ from (S)-(-)-glycidol.

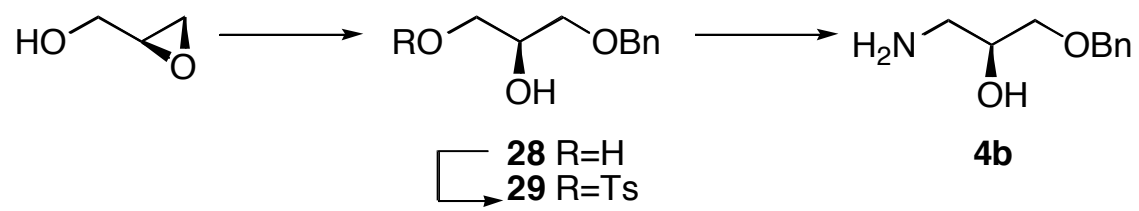

(2S)-3-Benzyloxy-1,2-propanol (28). ${ }^{5} \mathrm{NaH}(6.1 \mathrm{~g}, 152 \mathrm{mmol}, 60 \mathrm{w} \%$ dispersion in oil) was washed twice with pentane and dried under vacuum. THF $(100 \mathrm{~mL})$ was added and the suspension cooled in an ice/water bath. Benzyl alcohol $(15.7 \mathrm{~mL}, 152 \mathrm{mmol})$ dissolved in THF $(50 \mathrm{~mL})$ was added dropwise at 0 ${ }^{\circ} \mathrm{C}$. The reaction mixture was stirred for $30 \mathrm{~min}$ and the (S)-(-)-glycidol (4.5 g, $\left.61 \mathrm{mmol}\right)$ dissolved in THF $(50 \mathrm{~mL})$ was added carefully. The reaction mixture was stirred over night and the reaction was quenched with $\mathrm{NaHCO}_{3}$ (sat., $100 \mathrm{~mL}$ ). The phases were separated and the organic layer washed with brine and dried over $\mathrm{MgSO}_{4}$. Evaporation gave $20 \mathrm{~g}$ of a yellow oil that was purified by flash chromatography on silica gel (EtOAc:pentane 5:1) to give pure diol 28 in $65 \%$ yield $(7.2 \mathrm{~g}, 40 \mathrm{mmol}) .{ }^{1} \mathrm{H} \mathrm{NMR}\left(400 \mathrm{MHz}, \mathrm{CDCl}_{3}\right) \delta$ 7.36-7.26 (m, 5H), $4.53(\mathrm{~s}, 3 \mathrm{H}), 3.89-3.84(\mathrm{~m}, 1 \mathrm{H}), 3.65$ (AB-dd, J=11.5, 3.7 Hz, 1H), 3.57 (AB-dd, $J=11.5,4.2 \mathrm{~Hz}, 1 \mathrm{H}), 3.53$ (AB-dd, $J=9.6,4.2 \mathrm{~Hz}, 1 \mathrm{H}), 3.51$ (AB-dd, $J=9.6,6.1 \mathrm{~Hz}, 1 \mathrm{H}), 2.91(\mathrm{~s}, 2 \mathrm{H}) ;{ }^{13} \mathrm{C}$ NMR (100 MHz, $\left.\mathrm{CDCl}_{3}\right) \delta 138.1,128.9,128.3,128.2,74.0,72.2,71.2,64.4$.

(2R)-3-Benzyloxy-2-hydroxypropyl 4-methylbenzenesulfonat $(29)^{6}$ was prepared from 28 as described for 27 and obtained in 91\% yield. ${ }^{1} \mathrm{H} \mathrm{NMR}\left(400 \mathrm{MHz}, \mathrm{CDCl}_{3}\right) \delta 7.79(\mathrm{~d}, \mathrm{~J}=8.3 \mathrm{~Hz}, 2 \mathrm{H})$, 7.36$7.20(\mathrm{~m}, 7 \mathrm{H}), 4.49(\mathrm{~s}, 2 \mathrm{H}), 4.14-3.96(\mathrm{~m}, 3 \mathrm{H}), 3.54-3.47(\mathrm{~m}, 2 \mathrm{H}), 2.48(\mathrm{~d}, J=5.6,1 \mathrm{H}), 2.43(\mathrm{~s}, 3 \mathrm{H}) ;{ }^{13} \mathrm{C}$ NMR $\left(100 \mathrm{MHz}, \mathrm{CDCl}_{3}\right) \delta 145.5,137.9,133.1,130.4,130.2,128.9,128.2,128.1,73.9,71.0,70.5,68.8$, 22.1.

(S)-1-Amino-3-(benzyloxy)propan-2-ol (4b). Tosylate 29 (3.0 g, 8.9 mmol) was dissolved in THF $(12 \mathrm{~mL})$ and ammonium hydroxide $\left(30 \%\right.$ in $\left.\mathrm{H}_{2} \mathrm{O}, 23 \mathrm{~mL}\right)$ was added. The reaction mixture was stirred in a sealed vessel for $24 \mathrm{~h}$ at $80{ }^{\circ} \mathrm{C}$. After cooling the THF was evaporated and the residue extracted with 
$\mathrm{NaOH}(20 \mathrm{~mL}, 2 \mathrm{M})$ and $\mathrm{Et}_{2} \mathrm{O}(60 \mathrm{~mL})$. The water phase was extracted with $\mathrm{Et}_{2} \mathrm{O}(3 * 60 \mathrm{~mL})$ and $\mathrm{CH}_{2} \mathrm{Cl}_{2}$ (1*60 mL). After drying $\left(\mathrm{Na}_{2} \mathrm{SO}_{4}\right)$ and evaporation of the solvent pure amino alcohol (4b) remained in $92 \%$ yield $(1.5 \mathrm{~g}, 8.2 \mathrm{mmol})$. The product was used without further purification. mp: $58-63{ }^{\circ} \mathrm{C} .{ }^{1} \mathrm{H}$ NMR

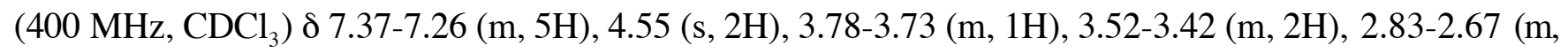
2H), 2.10 (br s, 3H); $\left.{ }^{13} \mathrm{C} \mathrm{NMR} \mathrm{(100} \mathrm{MHz,} \mathrm{CDCl}_{3}\right) \delta 138.5,128.9,128.21,128.19,73.9,73.0,71.5,44.8$; one signal is not visible; IR (neat): $3363(\mathrm{br}), 1332,1101 \mathrm{~cm}^{-1} ;[\alpha]_{\mathrm{D}}-5.1\left(c 1.00, \mathrm{CH}_{3} \mathrm{Cl}\right)$; HRMS (FAB+) calcd for $\mathrm{C}_{10} \mathrm{H}_{16} \mathrm{NO}_{2}(\mathrm{M}+\mathrm{H})$ : 182.1181 , found: 182.1181 .

\section{Experimental data for compounds 30a, 30b, 18a and $18 \mathrm{~b}$.}

Scheme S4. Preparation of $\mathbf{1 8 a}$ and $\mathbf{1 8 b}$ from dimethyl malonate.

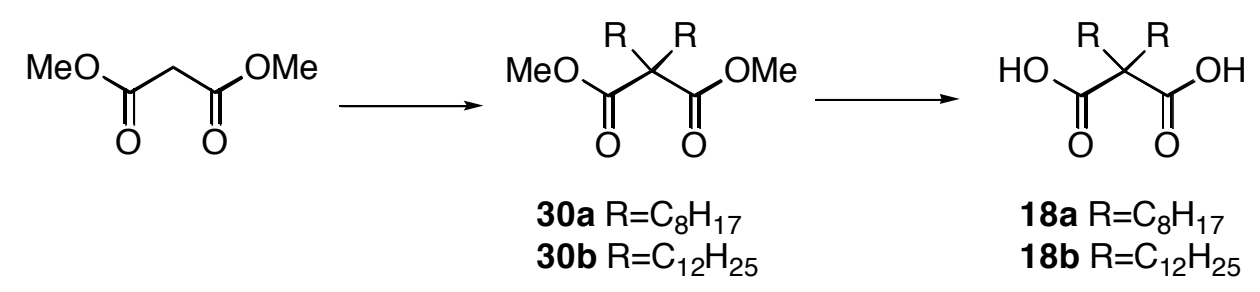

General procedure for the alkylation of dimethyl malonate. Dimethyl 2,2-Didodeylmalonate (30b) A solution of KHMDS in THF (15.1 mL, $22.7 \mathrm{mmol})$ was added dropwise to a solution of dimethyl malonate $(3.0 \mathrm{~g}, 22.7 \mathrm{mmol})$ in $\mathrm{THF}(150 \mathrm{~mL})$ at $0{ }^{\circ} \mathrm{C}$. When the addition was complete the turbid mixture was stirred for $15 \mathrm{~min}$. Dodecyl bromide $(5.5 \mathrm{~mL}, 22.9 \mathrm{mmol})$ was added and the reaction mixture refluxed for $10 \mathrm{~h}$. The temperature was lowered to $0{ }^{\circ} \mathrm{C}$, a second equiv. of KHMDS was added (15.1 mL, 22.7 mmol). The mixture was left for $15 \mathrm{~min}$. and dodecyl bromide $(5.5 \mathrm{~mL}, 22.9 \mathrm{mmol})$ was added. The mixture was allowed to reflux for $10 \mathrm{~h}$, then cooled and diluted with $\mathrm{Et}_{2} \mathrm{O}$. The organic phase was washed three times with $\mathrm{NaOH}(2 \mathrm{M})$ and two times with $\mathrm{HCl}$, then with brine. After drying $\left(\mathrm{MgSO}_{4}\right)$ and evaporation of the solvent, $10.2 \mathrm{~g}$ of a crude oil remained. The major part of the remaining dodecyl bromide and mono-alkylated product was removed by distillation under reduced pressure $\left(0.1\right.$ torr, $\left.100-200{ }^{\circ} \mathrm{C}\right)$ and the rest was purified by flash chromatography (rp C18 silica, $\mathrm{MeCN}$ ). This afforded 23\% of pure 30 b (2.5 g, $5.2 \mathrm{mmol}) .{ }^{1} \mathrm{H}$ NMR (400 MHz, $\mathrm{CDCl}_{3}$ ) $\delta 3.70(\mathrm{~s}, 6 \mathrm{H}), 1.88-1.84(\mathrm{~m}, 4 \mathrm{H}), 1.32-1.25$ (m, 36H), 1.14- 
$1.08(\mathrm{~m}, 4 \mathrm{H}), 0.88(\mathrm{t}, J=6.9 \mathrm{~Hz}, 6 \mathrm{H}) ;{ }^{13} \mathrm{C} \mathrm{NMR}\left(100 \mathrm{MHz}, \mathrm{CDCl}_{3}\right) \delta$ 172.5, 57.7, 52.2, 32.4, 31.9, 29.80, 29.66, 29.63, 29.62, 29.5, 29.3, 29.3, 24.0, 22.7, 14.1; IR (neat): $1738 \mathrm{~cm}^{-1}$; HRMS (FAB+) calcd for $\mathrm{C}_{29} \mathrm{H}_{57} \mathrm{O}_{4}(\mathrm{M}+\mathrm{H}): 469.4257$, found: 469.4251

Dimethyl 2,2-Dioctylmalonate $(30 a)^{7}$ was prepared from dimethyl malonate and octyl bromide as described for 30b and obtained in $51 \%$ yield. ${ }^{1} \mathrm{H}$ NMR $\left(400 \mathrm{MHz}, \mathrm{CDCl}_{3}\right) \delta 3.67(\mathrm{~s}, 6 \mathrm{H}), 1.84-1.80(\mathrm{~m}$, $4 \mathrm{H}), 1.27-1.22(\mathrm{~m}, 20 \mathrm{H}), 1.12-1.05(\mathrm{~m}, 4 \mathrm{H}), 0.84(\mathrm{t}, J=6.9 \mathrm{~Hz}, 6 \mathrm{H}) ;{ }^{13} \mathrm{C} \mathrm{NMR}\left(100 \mathrm{MHz}, \mathrm{CDCl}_{3}\right) \delta 173.3$, $58.1,52.6,32.9,32.3,30.2,29.7,29.6,24.4,23.1,14.5$

General procedure for the hydrolysis of malonic ester (30) to acid (18). 2,2-Dioctylmalonic acid (18a). ${ }^{8}$ Carboxylic ester 30a $(2.26 \mathrm{~g}, 6.3 \mathrm{mmol})$ was dissolved in $\mathrm{EtOH}(20 \mathrm{~mL})$ and an aq. solution of $\mathrm{KOH}(50 \mathrm{~mL}, 1.2 \mathrm{M})$ was added. The solution was refluxed for $100 \mathrm{~h}$, and then cooled using an external ice bath, and the excess $\mathrm{KOH}$ was neutralized by the addition of strongly acidic ion exchange resin (Amberlyst 15). The product precipitated as a white solid that was dissolved by the addition of $\mathrm{Et}_{2} \mathrm{O}$, and the resin was filtered off. The solvent was evaporated to give the product as a white powder in $94 \%$ yield (1.95 g, $5.9 \mathrm{mmol}) .{ }^{1} \mathrm{H}$ NMR (400 MHz, $\mathrm{CDCl}_{3}$ ) $\delta 3.70$ (s, 6H), 1.88-1.84 (m, 4H), 1.32-1.25 (m, 36H), 1.14-1.08 (m, 4H), $0.88(\mathrm{t}, J=6.9 \mathrm{~Hz}, 6 \mathrm{H}) ;{ }^{13} \mathrm{C} \mathrm{NMR}\left(100 \mathrm{MHz}, \mathrm{CDCl}_{3}\right) \delta$ 177.6, 57.8, 34.7, 31.8, 29.6, 29.20, 29.17, 24.6, 22.6, 14.1 .

2,2-Didodecylmalonic acid (18b) ${ }^{\mathbf{9}}$ was prepared from $\mathbf{3 0 b}$ as described for $\mathbf{1 8 a}$ and obtained in $73 \%$ yield. ${ }^{1} \mathrm{H}$ NMR $\left(400 \mathrm{MHz}, \mathrm{CDCl}_{3}\right) \delta$ 1.97-1.93 (m, 4H), 1.32-1.06 (m, 40H), $0.88(\mathrm{t}, J=6.9 \mathrm{~Hz}, 6 \mathrm{H}) ;{ }^{13} \mathrm{C}$ NMR $\left(100 \mathrm{MHz} \mathrm{CDCl}_{3}\right) \delta 178.3,58.3,35.2,32.4,30.15,30.10,30.10,30.07,30.02,29.8,25.0,23.1$, 14.4. 


\section{Experimental data for compounds 31a, 31b, 32a, 32b, 2a and $2 \mathrm{~b}$.}

Scheme S4. Preparation of surfactants $\mathbf{2 a}$ and $\mathbf{2 b}$.

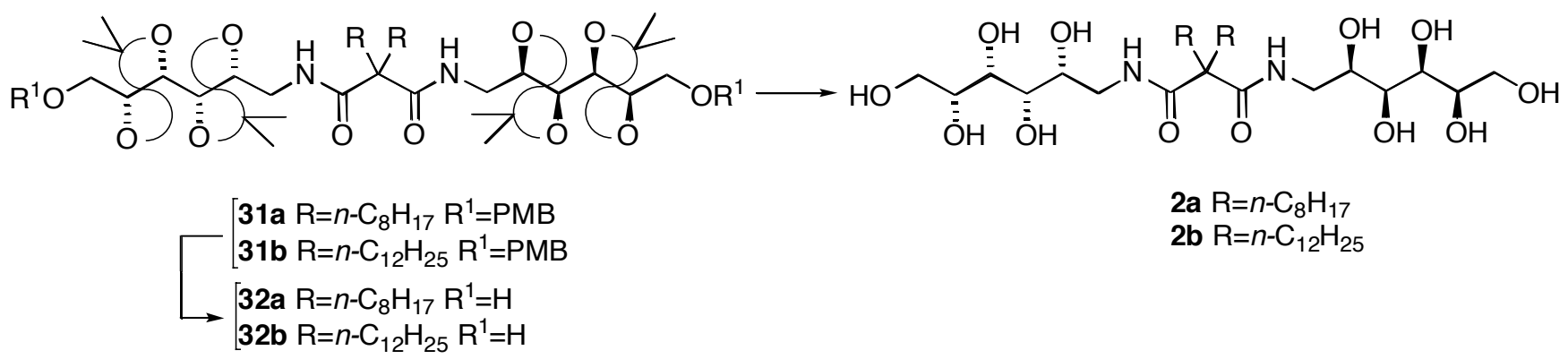

$N^{1}, N^{3}$-Bis-(1-deoxy-2,3:4,5-di- $O$-isopropylidene-D-iditol-6-(4-methoxybenzoate))-2,2-

dioctylmalonamide (31a) was prepared from 18a and $\mathbf{1 4}$ as described for 20a and obtained in 59\% yield. ${ }^{1} \mathrm{H}$ NMR $\left(500 \mathrm{MHz}, \mathrm{CDCl}_{3}\right) \delta 7.44(\mathrm{t}, J=5.7 \mathrm{~Hz}, 2 \mathrm{H}), 7.28(\mathrm{~d}, J=8.4 \mathrm{~Hz}, 4 \mathrm{H}), 6.90(\mathrm{~d}, J=8.4 \mathrm{~Hz}$, 4H), 4.54 (AB-d, $J=11.7 \mathrm{~Hz}, 2 \mathrm{H}), 4.52$ (AB-d, $J=11.7 \mathrm{~Hz}, 2 \mathrm{H}), 4.31-4.27$ (m, 2H), 4.17-4.14 (m, 2H), 3.88 (dd, J=8.3, 3.2 Hz, 2H); 3,82 (s, 6H), 3.69 (dd, J=8.3, 3.2 Hz, 2H), $3.61(\mathrm{dd}, J=10.3,5.5 \mathrm{~Hz}, 2 \mathrm{H}), 3.58-$ $3.52(\mathrm{~m}, 4 \mathrm{H}), 3.46(\mathrm{td}, J=14.2,5.7 \mathrm{~Hz}, 2 \mathrm{H}), 1.84-1.81(\mathrm{~m}, 4 \mathrm{H}), 1.44-1.43(\mathrm{~m}, 18 \mathrm{H}), 1.40(\mathrm{~s}, 6 \mathrm{H}), 1.30-1.18$ (m, 24H), 0.89 (t, J=6.7 Hz, 6H); ${ }^{13} \mathrm{C}$ NMR $\left(125 \mathrm{MHz}, \mathrm{CDCl}_{3}\right) \delta 173.4,159.3,130.0,129.4,113.8,110.7$, 109.4, 77.23, 77.18, 76.2, 75.8, 73.2, 70.1, 57.1, 55.3, 40.3, 37.6, 31.8, 29.9, 29.4, 29.3, 27.4, 27.2, 26.68, 26.66, 25.0, 22.6, 14.1; IR (neat): 3370, 1666, $1514 \mathrm{~cm}^{-1} ;[\alpha]_{\mathrm{D}}+14.9\left(c\right.$ 0.70, $\left.\mathrm{CHCl}_{3}\right)$; $\mathrm{HRMS}(\mathrm{FAB}+)$ calcd for $\mathrm{C}_{59} \mathrm{H}_{94} \mathrm{~N}_{2} \mathrm{O}_{14} \mathrm{Na}(\mathrm{M}+\mathrm{Na})$ : 1077.6603 , found: 1077.6599 .

\section{$N^{1}, N^{3}$-Bis-(1-deoxy-2,3:4,5-di- $O$-isopropylidene-D-iditol-6-(4-methoxybenzoate))-2,2-}

didodecylmalonamide (31b) was prepared from $\mathbf{1 8 b}$ and $\mathbf{1 4}$ as described for $\mathbf{2 0 a}$ and obtained in $57 \%$ yield. ${ }^{1} \mathrm{H}$ NMR $\left(500 \mathrm{MHz}, \mathrm{CDCl}_{3}\right) \delta 7.44(\mathrm{t}, J=5.6 \mathrm{~Hz}, 2 \mathrm{H}), 7.28(\mathrm{~d}, J=8.5 \mathrm{~Hz}, 4 \mathrm{H}), 6.90(\mathrm{~d}, J=8.5 \mathrm{~Hz}$, 4H), 4.54 (AB-d, $J=11.8 \mathrm{~Hz}, 2 \mathrm{H}), 4.52$ (AB-d, $J=11.8 \mathrm{~Hz}, 2 \mathrm{H}), 4.31-4.27$ (m, 2H), 4.17-4.14 (m, 2H), 3.88 (dd, J=8.3, 3.2 Hz, 2H), 3.82 (s, 6H), 3.69 (dd, J=8.3, 3.2 Hz, 2H), 3.61 (dd, J=10.3, 5.5 Hz, 2H), 3.58$3.52(\mathrm{~m}, 4 \mathrm{H}), 3.46 \mathrm{td}, J=14.2,5.6 \mathrm{~Hz}, 2 \mathrm{H}), 1.84-1.81(\mathrm{~m}, 4 \mathrm{H}), 1.44-1.43(\mathrm{~m}, 18 \mathrm{H}), 1.40(\mathrm{~s}, 6 \mathrm{H}), 1.33-1.17$ (m, 40H), $0.90(\mathrm{t}, J=7.0 \mathrm{~Hz}, 6 \mathrm{H}) ;{ }^{13} \mathrm{C}$ NMR $\left(125 \mathrm{MHz}, \mathrm{CDCl}_{3}\right) \delta 173.4,159.3,130.0,129.4,113.8,109.7$, 
$109.5,77.23,77.18,76.2,75.8,73.2,70.1,57.1,55.3,40.3,37.5,32.0,30.1,29.68,29.70,29.70,29.46$, 29.37, 27.4, 27.2, 26.69, 26.67, 25.0, 22.7, 14.1; one signal is not visible; IR (neat) 2926, 1667, 1514, $\mathrm{cm}^{-1}$; $[\alpha]_{\mathrm{D}}+12.9\left(c \quad 0.70, \mathrm{CHCl}_{3}\right)$; HRMS $(\mathrm{FAB}+)$ calcd for $\mathrm{C}_{67} \mathrm{H}_{110} \mathrm{~N}_{2} \mathrm{O}_{14} \mathrm{Na}(\mathrm{M}+\mathrm{Na})$ : 1089.7855, found: 1089.7855 .

$N^{1}, N^{3}$-Bis-(1-deoxy-2,3:4,5-di- $O$-isopropylidene-D-iditol)-2,2-dioctylmalonamide (32a) was prepared from 31a as described for 21a and obtained in 98\% yield. ${ }^{1} \mathrm{H}$ NMR: $\left(500 \mathrm{MHz}, \mathrm{CDCl}_{3}\right) \delta 7.42$ (t, J=5.4 Hz, 2H), 4.21-4.17 (m, 4H), 4.02 (dd, J=8.3, 2.7 Hz, 2H), 3.80 (dd, J=11.8, 4.6 Hz, 2H), 3.77$3.72(\mathrm{~m}, 4 \mathrm{H}), 3.62-3.52(\mathrm{~m}, 4 \mathrm{H}), 2.59$ (br s $2 \mathrm{H}), 1.84-1.81(\mathrm{~m}, 4 \mathrm{H}), 1.46-1.44(\mathrm{~m}, 18 \mathrm{H}), 1.42(\mathrm{~s}, 6 \mathrm{H})$, 1.32-1.17 (m, 24H), 0.89 (t, $J=7.0 \mathrm{~Hz}, 6 \mathrm{H}) ;{ }^{13} \mathrm{C} \mathrm{NMR}\left(125 \mathrm{MHz}, \mathrm{CDCl}_{3}\right) \delta 173.7,109.6,109.4,77.2$, 76.9, 76.3, 75.9, 62.3, 57.4, 40.0, 37.1, 31.8, 29.9, 29.31, 29.23, 27.31, 27.25, 26.6, 26.5, 24.9, 22.6, 14.1; IR (neat): 2928, 1661, $1530 \mathrm{~cm}^{-1} ;[\alpha]_{\mathrm{D}}-1.1\left(c 0.80, \mathrm{CHCl}_{3}\right)$; HRMS $(\mathrm{FAB}+)$ calcd for $\mathrm{C}_{43} \mathrm{H}_{78} \mathrm{~N}_{2} \mathrm{O}_{12} \mathrm{Na}$ $(\mathrm{M}+\mathrm{Na}): 837.5452$, found: 837.5453 .

\section{$N^{1}, N^{3}$-Bis-(1-deoxy-2,3:4,5-di- $O$-isopropylidene-D-iditol)-2,2-didodecylmalonamide (32b) was} prepared from $\mathbf{3 1 b}$ as described for $\mathbf{2 1 a}$ and obtained in $93 \%$ yield. ${ }^{1} \mathrm{H}$ NMR $\left(500 \mathrm{MHz}, \mathrm{CDCl}_{3}\right) \delta 7.42(\mathrm{t}$, $J=5.8 \mathrm{~Hz}, 2 \mathrm{H}), 4.21-4.17(\mathrm{~m}, 4 \mathrm{H}), 4.01$ (dd, $J=8.3,2.3 \mathrm{~Hz}, 2 \mathrm{H}), 3.80$ (dd, J=11.9, $4.7 \mathrm{~Hz}, 2 \mathrm{H}), 3.77-3.71$ (m, 4H), 3.62-3.52 (m, 4H), 2.60 (br s, 2H), 1.84-1.81 (m, 4H), 1.45-1.44 (m, 18H), 1.42 (s, 6H), $1.33-$

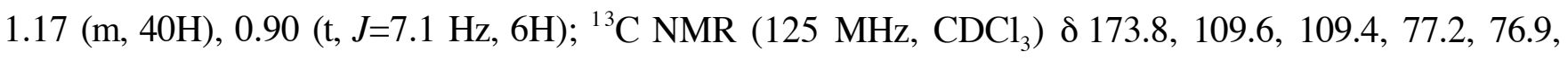
76.3, 62.3, 57.3, 39.9, 37.1, 32.0, 29.9, 29.64, 29.64, 29.62, 29.61, 29.40, 29.34, 27.31, 27.25, 26.63, 26.55, 24.9, 22.7, 14.1; one signal is not visible; IR (neat): 2926, 1663, $1531 \mathrm{~cm}^{-1} ;[\alpha]_{\mathrm{D}}-1.0\left(c 1.00, \mathrm{CHCl}_{3}\right)$; HRMS (FAB+) calcd for $\mathrm{C}_{51} \mathrm{H}_{94} \mathrm{~N}_{2} \mathrm{O}_{12} \mathrm{Na}(\mathrm{M}+\mathrm{Na})$ : 949.6704, found: 949.6714.

$N^{1}, N^{3}$-Bis-(1-deoxy-D-iditol)-2,2-dioctylmalonamide (2a) was prepared from 32a as described for 1a and obtained as a white powder in $67 \%$ yield. ${ }^{1} \mathrm{H}$ NMR (500 MHz, CD $\left.\mathrm{OD}\right) \delta$ 3.91-3.88 (m, 2H), 3.80-3.77 (m, 4H), 3.71-3.64 (m, 6H), 3.49 (dd, J=13.6, $5.1 \mathrm{~Hz}, 2 \mathrm{H}), 3.39-3.35(\mathrm{~m}, 2 \mathrm{H}), 1.92-1.84(\mathrm{~m}$, $4 \mathrm{H}), 1.35-1.28(\mathrm{~m}, 4 \mathrm{H}), 1.21-1.17(\mathrm{~m}, 20 \mathrm{H}), 0.92(\mathrm{t}, J=6.9 \mathrm{~Hz}, 6 \mathrm{H}) ;{ }^{13} \mathrm{C} \mathrm{NMR}(100 \mathrm{MHz}, \mathrm{MeOH})$ 
$\delta 176.4,73.7,72.3,73.2,71.7,64.9,59.3,44.2,36.4,33.4,31.4,30.8,26.0,24.1,14.9$; one signal is not visible; IR (neat): 3350 (br), 1642, $1530 \mathrm{~cm}^{-1} ;[\alpha]_{\mathrm{D}}+3.0$ (c 1.00, MeOH); HRMS (FAB+) calcd for $\mathrm{C}_{31} \mathrm{H}_{63} \mathrm{~N}_{2} \mathrm{O}_{12}(\mathrm{M}+\mathrm{H}): 655.4381$, found: 655.4381 .

$N^{1}, N^{3}$-Bis-(1-deoxy-D-iditol)-2,2-didodecylmalonamide (2b) was prepared form 32b as described for $1 \mathrm{a}$ and obtained as a white powder in $87 \%$ yield. ${ }^{1} \mathrm{H}$ NMR (500 MHz, $\mathrm{CD}_{3} \mathrm{OD}: \mathrm{CDCl}_{3}$ 1:1, $313 \mathrm{~K}$ ) ઈ 3.79-3.76 (m, 2H), 3.71-3.66 (m, 4H), 3.58 (d, J=5.4Hz, 4H), 3.53 (t, J=3.8 Hz, 2H), 3.40 (dd, J=13.7, $5.1 \mathrm{~Hz}, 2 \mathrm{H}), 3.29-3.24(\mathrm{~m}, 2 \mathrm{H}), 1.78-1.72(\mathrm{~m}, 4 \mathrm{H}), 1.94-1.17(\mathrm{~m}, 36 \mathrm{H}), 1.08-1.06(\mathrm{~m}, 4 \mathrm{H}), 0.78(\mathrm{t}, J=6.9$ $\mathrm{Hz}, 6 \mathrm{H}) ;{ }^{13} \mathrm{C}$ NMR (125 MHz, MeOH:CDCl 3 1:1) $\delta$ 174.7, 72.4, 72.3, 72.0, 70.5, 63.7, 58.1, 43.0, 34.5, 32.1, 30.2, 29.9, 29.8, 29.8, 29.6, 29.5, 24.6, 22.8, 13.9; one signal is not visible; IR (neat): 3326 (br), 1644 , $1529 \mathrm{~cm}^{-1}$; HRMS (FAB+) calcd for $\mathrm{C}_{39} \mathrm{H}_{79} \mathrm{~N}_{2} \mathrm{O}_{12}(\mathrm{M}+\mathrm{H}): 767.5633$, found: 767.5618.

\section{Analytical data for compounds ent-21a, ent-21b, ent-1a and ent-1b.}

$N^{1}, N^{3}$-Bis-(1-deoxy-2,3:4,5-di- $O$-isopropylidene-D-galactitol)-2,2-dioctylmalonamide (ent-21a) was prepared from 18a and $\mathbf{1 7}$ as described for 20a and obtained as a clear oil in $45 \%$ yield. ${ }^{1} \mathrm{H}$ NMR $\left(500 \mathrm{MHz}, \mathrm{CDCl}_{3}\right) \delta 7.59(\mathrm{dd}, J=6.6,4.3 \mathrm{~Hz}, 2 \mathrm{H}), 4.09-4.06(\mathrm{~m}, 4 \mathrm{H}), 3.87-3.73(\mathrm{~m}, 8 \mathrm{H}), 3.66(\mathrm{t}, J=7.8$ Hz, 2H), 3.51 (td, J=13.9, $4.3 \mathrm{~Hz}, 2 \mathrm{H}), 2.30$ (dd, J=8.6, $4.4 \mathrm{~Hz}, 2 \mathrm{H}), 1.87-1.80$ (m, 4H), 1.47 (s, 6H), 1.45 $(\mathrm{s}, 6 \mathrm{H}), 1.43(\mathrm{~s}, 6 \mathrm{H}), 1.39(\mathrm{~s}, 6 \mathrm{H}), 1.32-1.17(\mathrm{~m}, 24 \mathrm{H}), 0.89(\mathrm{t}, J=6.9 \mathrm{~Hz}, 6 \mathrm{H}) ;{ }^{13} \mathrm{C} \mathrm{NMR}(125 \mathrm{MHz}$, $\left.\mathrm{CDCl}_{3}\right): \delta 173.2,110.1,109.8,80.9,79.7,79.2,78.6,62.5,57.1,40.8,37.5,31.8,29.9,29.4,29.3,27.1$, 27.0, 26.9, 26.8, 24.9, 22.6, 14.1; IR (neat): 3389 (br), 1666, $1530 \mathrm{~cm}^{-1} ;[\alpha]_{\mathrm{D}}+6.9\left(c 0.90, \mathrm{CHCl}_{3}\right)$; HRMS $(\mathrm{FAB}+)$ calcd for $\mathrm{C}_{43} \mathrm{H}_{79} \mathrm{~N}_{2} \mathrm{O}_{12} \mathrm{Na}(\mathrm{M}+\mathrm{H}): 815.5633$, found: 815.5641.

$N^{1}, N^{3}$-Bis-(1-deoxy-2,3:4,5-di- $O$-isopropylidene-D-galactitol)-2,2-didocecylmalonamide (ent21b) was prepared from $\mathbf{1 8 b}$ and 17 as described for 20a and obtained as a clear oil in $45 \%$ yield. ${ }^{1} \mathrm{H}$ NMR (400 MHz, $\left.\mathrm{CDCl}_{3}\right) \delta 7.55(\mathrm{dd}, J=6.52,4.32 \mathrm{~Hz}, 2 \mathrm{H}), 4.07-4.03(\mathrm{~m}, 4 \mathrm{H}), 3.85-3.69$ (m, 8H), $3.64(\mathrm{t}$, $J=8.0 \mathrm{~Hz}, 2 \mathrm{H}), 3.49(\mathrm{td}, J=13.9,4.4 \mathrm{~Hz}, 2 \mathrm{H}), 2.36(\mathrm{dd}, J=7.86,4.30 \mathrm{~Hz}, 2 \mathrm{H}), 1.85-1.77(\mathrm{~m}, 4 \mathrm{H}), 1.44(\mathrm{~s}$, 
6H), $1.43(\mathrm{~s}, 6 \mathrm{H}), 1.40(\mathrm{~s}, 6 \mathrm{H}), 1.37(\mathrm{~s}, 6 \mathrm{H}), 1.23-.118(\mathrm{~m}, 40 \mathrm{H}), 0.88(\mathrm{t}, J=13.7,6.9 \mathrm{~Hz}, 6 \mathrm{H}) ;{ }^{13} \mathrm{C}$ NMR $\left(100 \mathrm{MHz}, \mathrm{CDCl}_{3}\right): \delta 173.6,110.5,110.2,81.3,80.2,79.6,79.0,62.9,57.5,41.2,37.9,32.3,30.3,30.1$, $30.1,30.1,29.9,29.8,27.53,27.50,27.3,27.2,25.4,23.1,14.5$; one signal is not visible; IR (neat): 3374 (br), 1667, 1530, cm $\mathrm{cm}^{-1}[\alpha]_{\mathrm{D}}+6.0\left(c\right.$ 1.09, $\left.\mathrm{CHCl}_{3}\right)$ HRMS $(\mathrm{FAB}+)$ calcd for $\mathrm{C}_{51} \mathrm{H}_{94} \mathrm{~N}_{2} \mathrm{O}_{12} \mathrm{Na}(\mathrm{M}+\mathrm{Na})$ : 949.6704, found: 949.6710 .

$N^{1}, N^{3}$-Bis-(1-deoxy-D-galactitol)-2,2-dioctylmalonamide ent-1a was prepared from ent-21a as described for 1a and obtained as a white powder in $99 \%$ yield. ${ }^{1} \mathrm{H}$ NMR (500 MHz, CD 3 OD) $\delta 3.98$ (br t, $J=6.4 \mathrm{~Hz}, 2 \mathrm{H}), 3.91$ (br t, $J=5.7 \mathrm{~Hz}, 2 \mathrm{H}), 3.66-3.63(\mathrm{~m}, 6 \mathrm{H}), 3.53$ (br d, $J=9.1 \mathrm{~Hz}, 2 \mathrm{H}), 3.45$ (dd, $J=13.5$, $5.7 \mathrm{~Hz}, 2 \mathrm{H}), 1.86-1.82(\mathrm{~m}, 4 \mathrm{H}), 1.31-1.28(\mathrm{~m}, 20 \mathrm{H}), 1.20-1.02(\mathrm{~m}, 4 \mathrm{H}), 0.88(\mathrm{t}, J=6.9 \mathrm{~Hz}, 6 \mathrm{H}) ;{ }^{13} \mathrm{C}$ NMR (125 MHz, MeOD): $\delta$ 176.1, 72.0, 71.9, 71.3, 70.0, 65.0, 58.8, 49.9, 44.2, 35.9, 33.1, 31.0, 30.4, 25.5, 23.8, 14.5; IR (neat): 3339 (br), 1627, $1439 \mathrm{~cm}^{-1} ;[\alpha]_{\mathrm{D}}-10.8\left(c 1.00, \mathrm{CHCl}_{3}\right)$; HRMS (FAB+) calcd for $\mathrm{C}_{31} \mathrm{H}_{63} \mathrm{~N}_{2} \mathrm{O}_{12}(\mathrm{M}+\mathrm{H}):$ 655.4381, found: 655.4380 .

$N^{1}, N^{3}$-Bis-(1-deoxy-D-galactitol)-2,2-didodecylmalonamide ent-1b was prepared form ent-21b as described for $1 \mathrm{a}$ and obtained as a white powder in 99\% yield. ${ }^{1} \mathrm{H}$ NMR (500 $\mathrm{MHz}, \mathrm{CD}_{3} \mathrm{OD}: \mathrm{CDCl}_{3} 1: 1$, 313 K) $\delta 3.97-3.90(\mathrm{~m}, 4 \mathrm{H}), 3.70-3.62(\mathrm{~m}, 6 \mathrm{H}), 3.52-3.47(\mathrm{~m}, 4 \mathrm{H}), 1.87-1.82(\mathrm{~m}, 4 \mathrm{H}), 1.32-1.12(\mathrm{~m}$, 40H), $0.88(\mathrm{t}, J=6.82 \mathrm{~Hz}, 6 \mathrm{H}) ;{ }^{13} \mathrm{C} \mathrm{NMR}\left(125 \mathrm{MHz}, \mathrm{MeOD}: \mathrm{CDCl}_{3}\right.$ 1:1, 313K): $\delta 174.6,71.2,70.9,70.8$, 69.1, 64.3, 58.1, 49.3, 43.2, 34.0, 32.1, 30.2, 29.89, 29.85, 29.85, 29.6, 29.5, 24.5, 22.8, 13.9; IR (neat): 3339 (br) 1627, $1439 \mathrm{~cm}^{-1}$; HRMS (FAB+) calcd for $\mathrm{C}_{39} \mathrm{H}_{79} \mathrm{~N}_{2} \mathrm{O}_{12}(\mathrm{M}+\mathrm{H}):$ 767.5633, found: 767.5635. 


\section{References:}

(1) Somfai, P.; Marchand, P.; Torsell, S.; Lindström, U. M. Tetrahedron 2003, 59, 1293-1299.

(2) Huntington, K. M.; Yi, T.; Wei, Y.; Pei, D. Biochemistry 2000, 39, 4543-4551.

(3) Lim, Z.-Y.; Thuring, J. W.; Holmes, A. B.; Manifava, M.; Ktistakis, N. T. J. Chem. Soc., Perkin Trans. 1 2002, 1067-1075.

(4) Wennerberg, J.; Olofsson, C.; Frejd, T. J. Org. Chem. 1998, 63, 33595-3598.

(5) Balint, J.; Egri, G.; Kolbert, A.; Dianoczky, C.; Fogassy, E.; Novak, L.; Poppe, L. Tetrahedron: Asymmetry 1999, 10, 4017-4028.

(6) Byun, H. S.; Bittman, R. Tetrahedron Lett. 1989, 30, 2751-4.

(7) Ishii, Y.; Iwahama, T.; Sakaguchi, S.; Nakano, T. PCT Int. Appl. Wo 2001066501, 2001.

(8) Deline, J. E.; Ott, M. M. PCT Int. Appl. Wo 2001002343, 2001.

(9) Chargaff, E. Ber. 1932, 65B, 745-54. 
7. NMR spectra for compounds1a, $\mathbf{b}$, ent-1a, $\mathbf{b}, \mathbf{2 a}, \mathbf{b}, \mathbf{4 a}, \mathbf{b}$, ent-6, 7, ent-7, 8-17, 20a, b, 21a, b, ent-21a, b, 22-24, 27, 30b, 31a, b, 32a, b. 

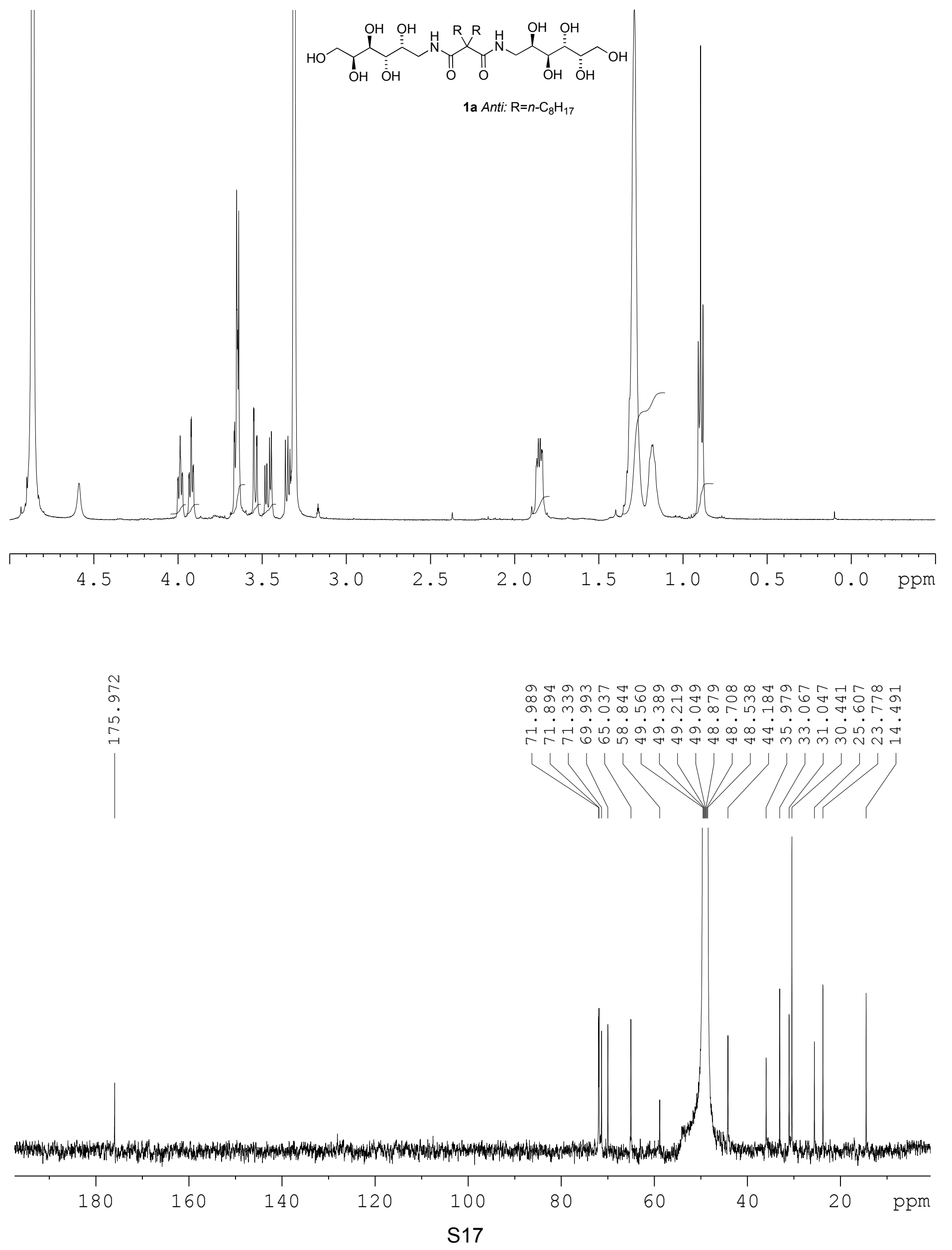

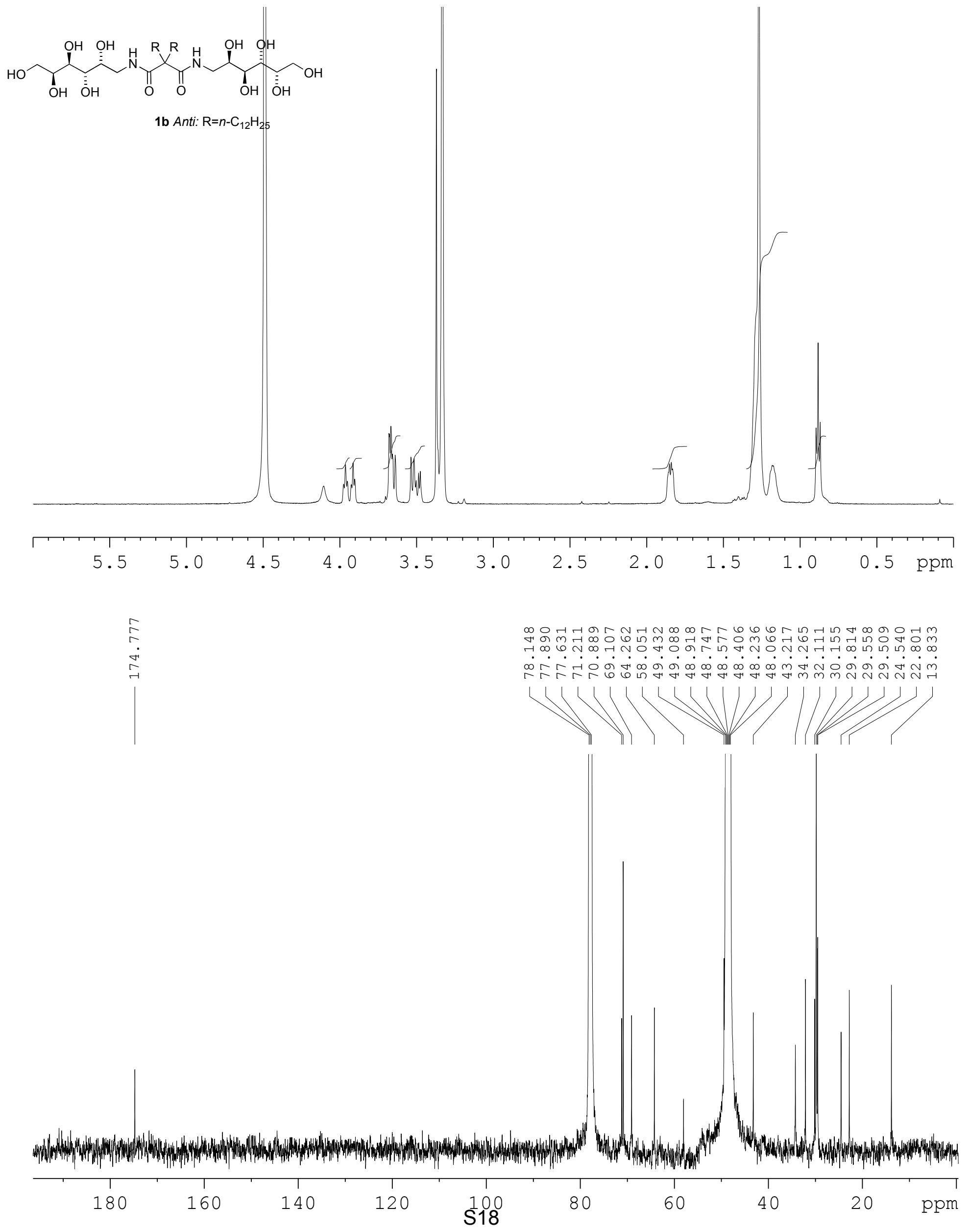

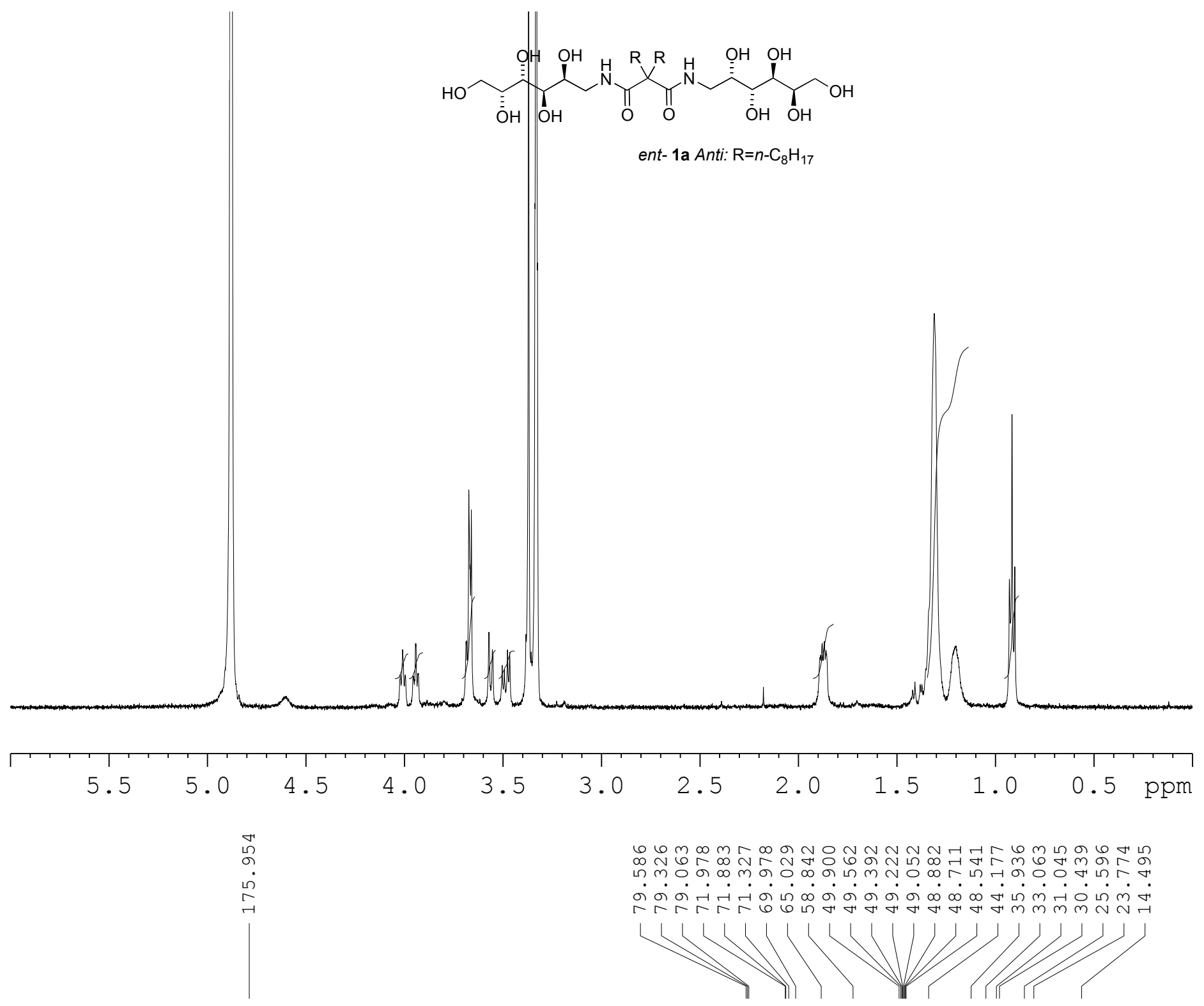

6 ๓ बंள

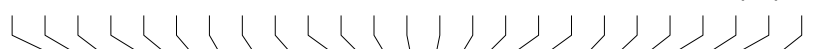
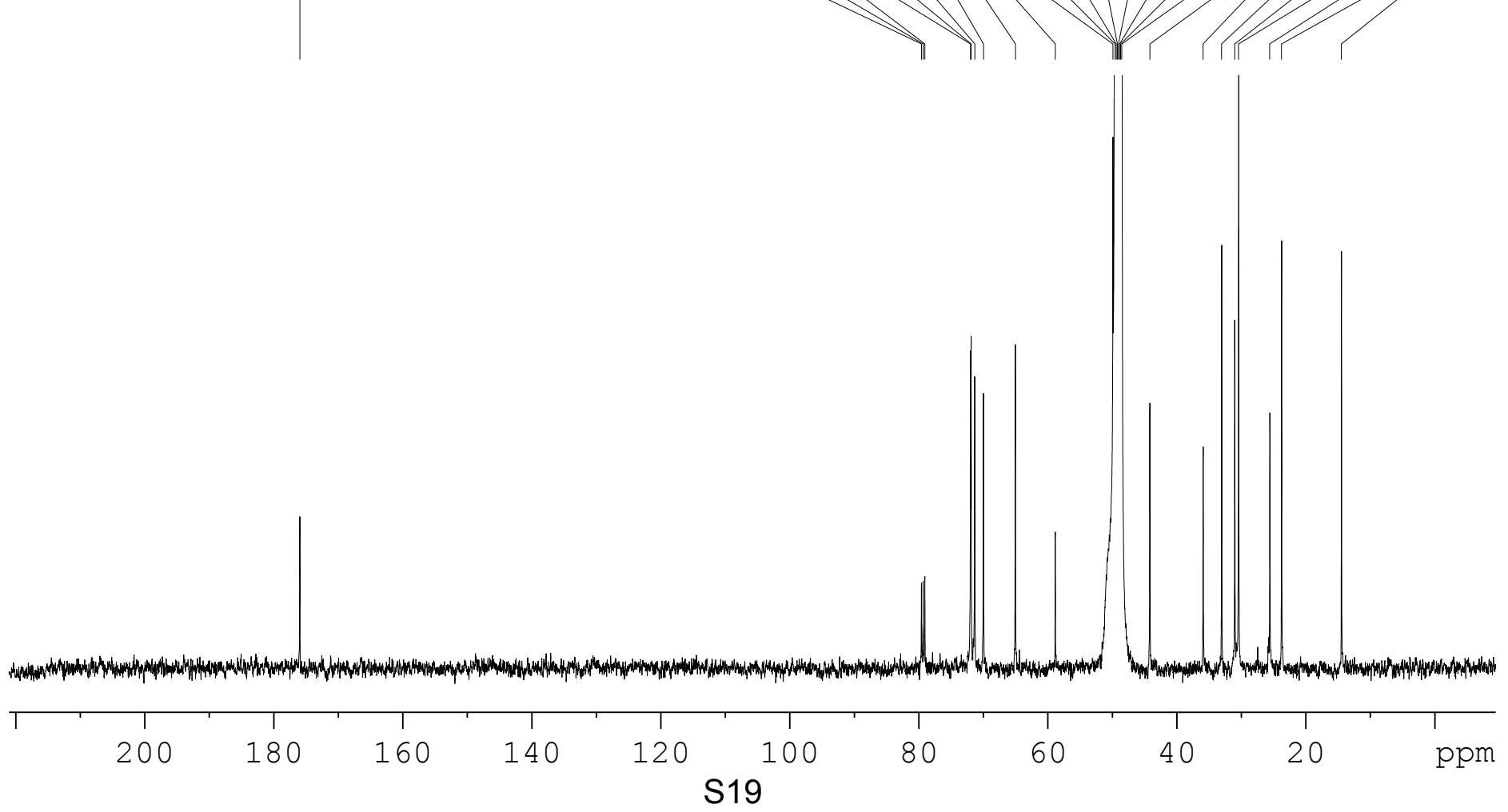

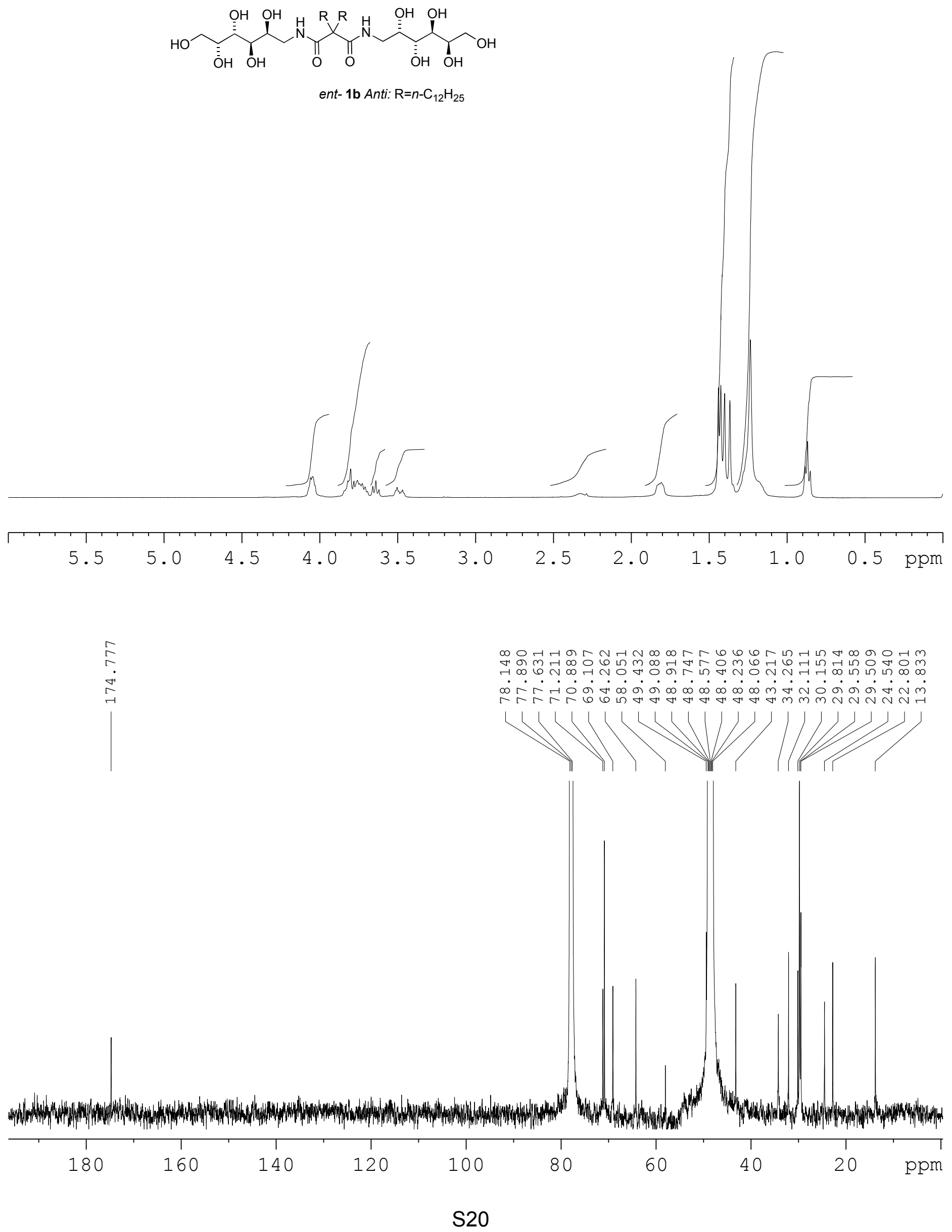

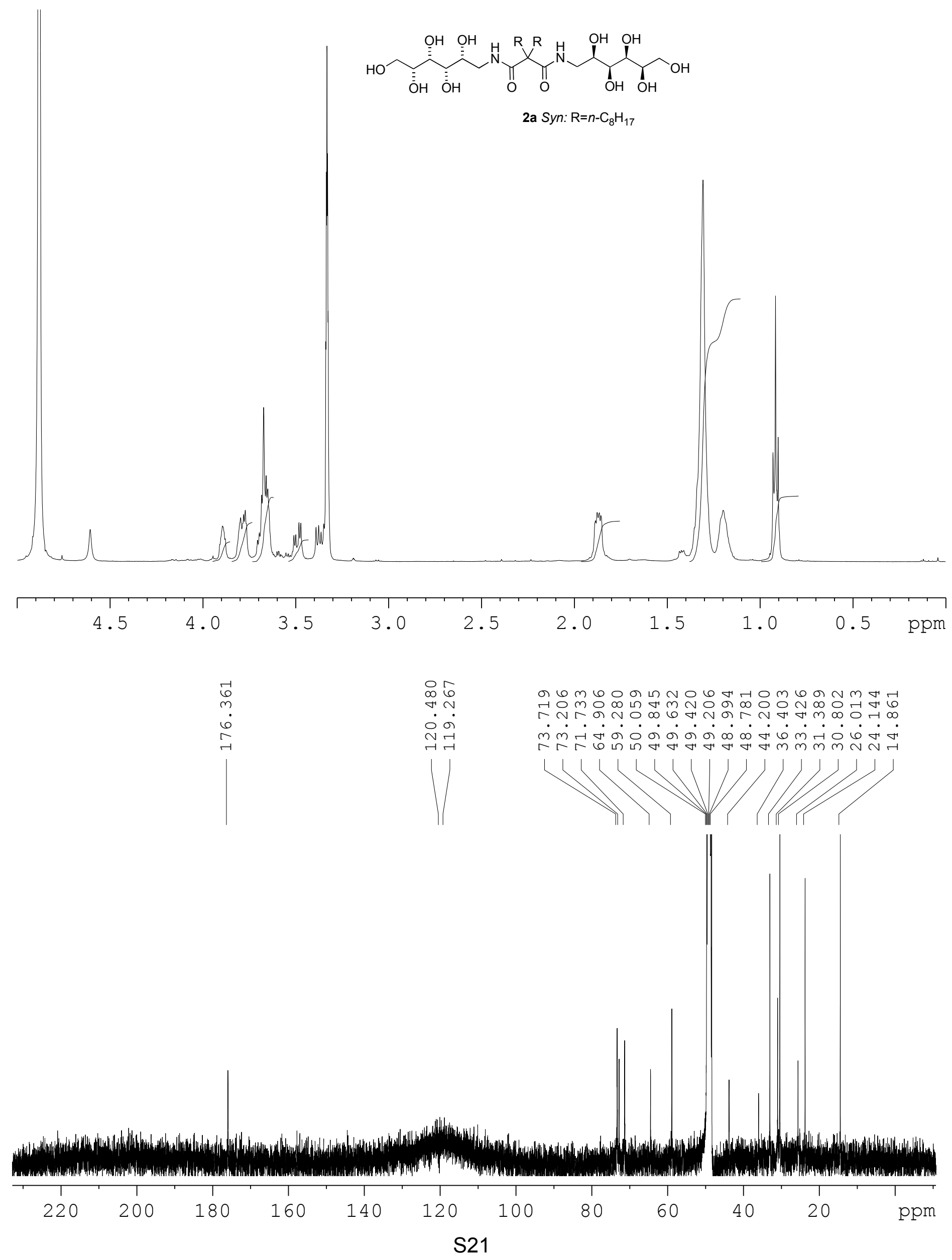


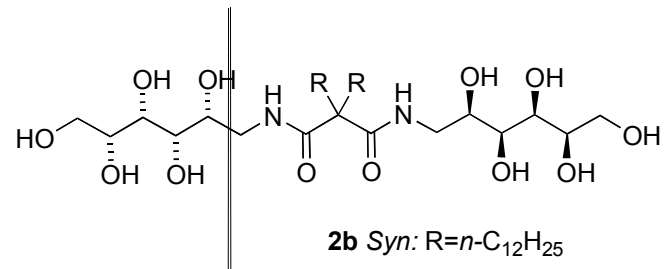

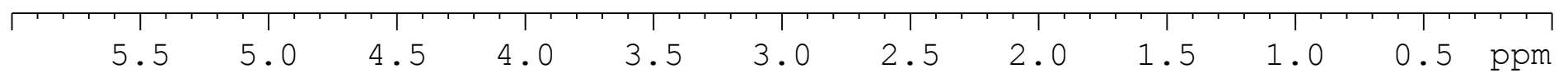

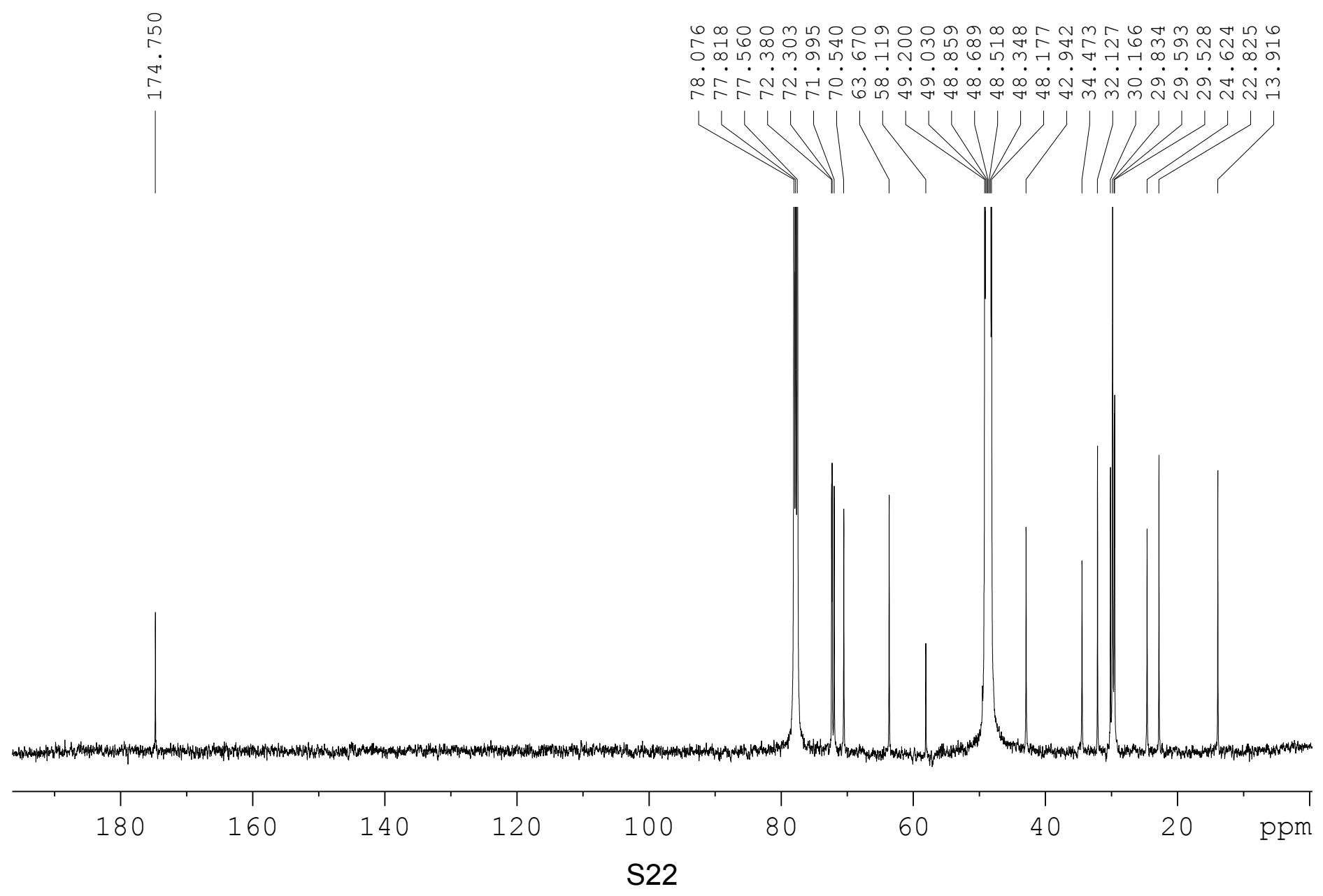




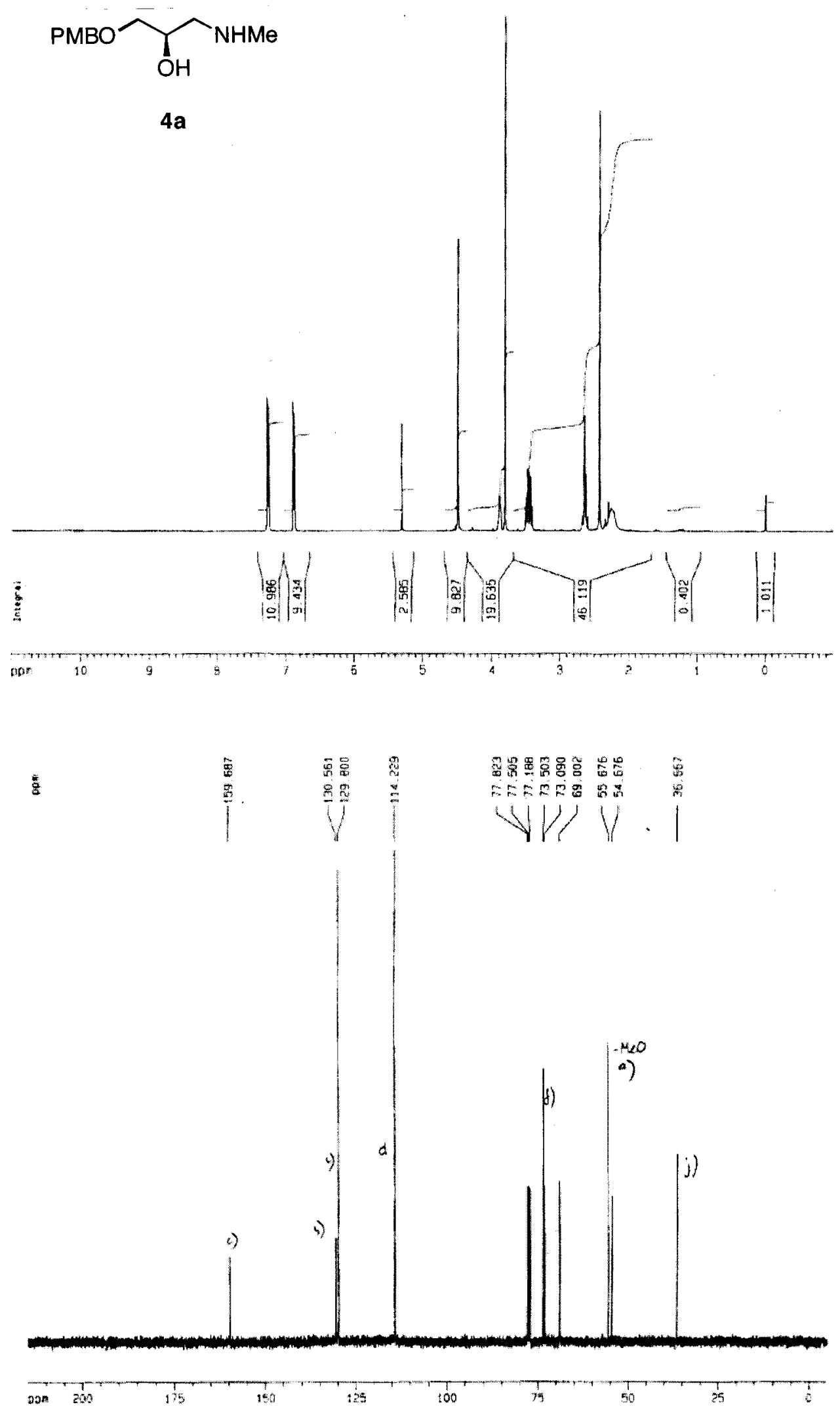




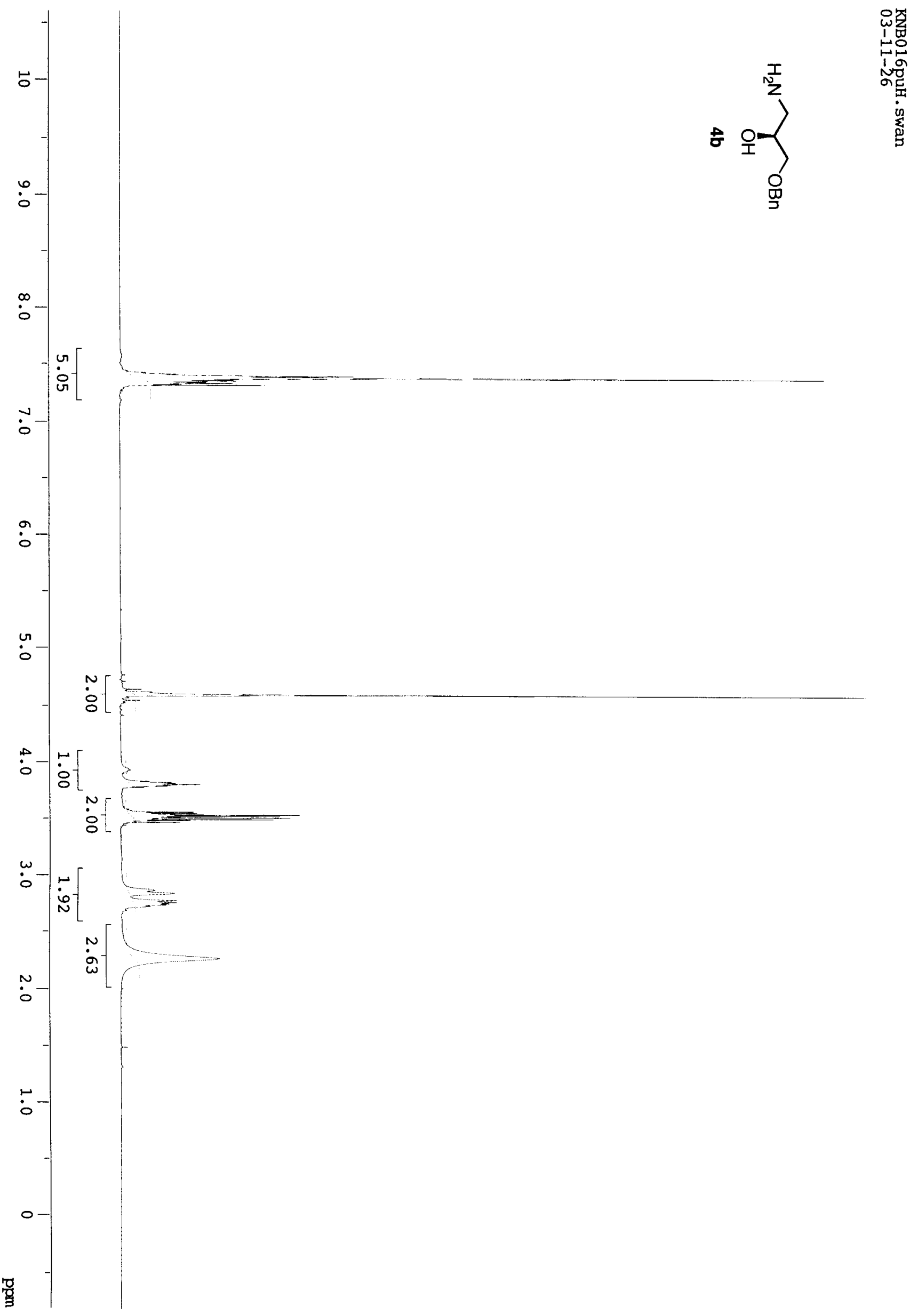



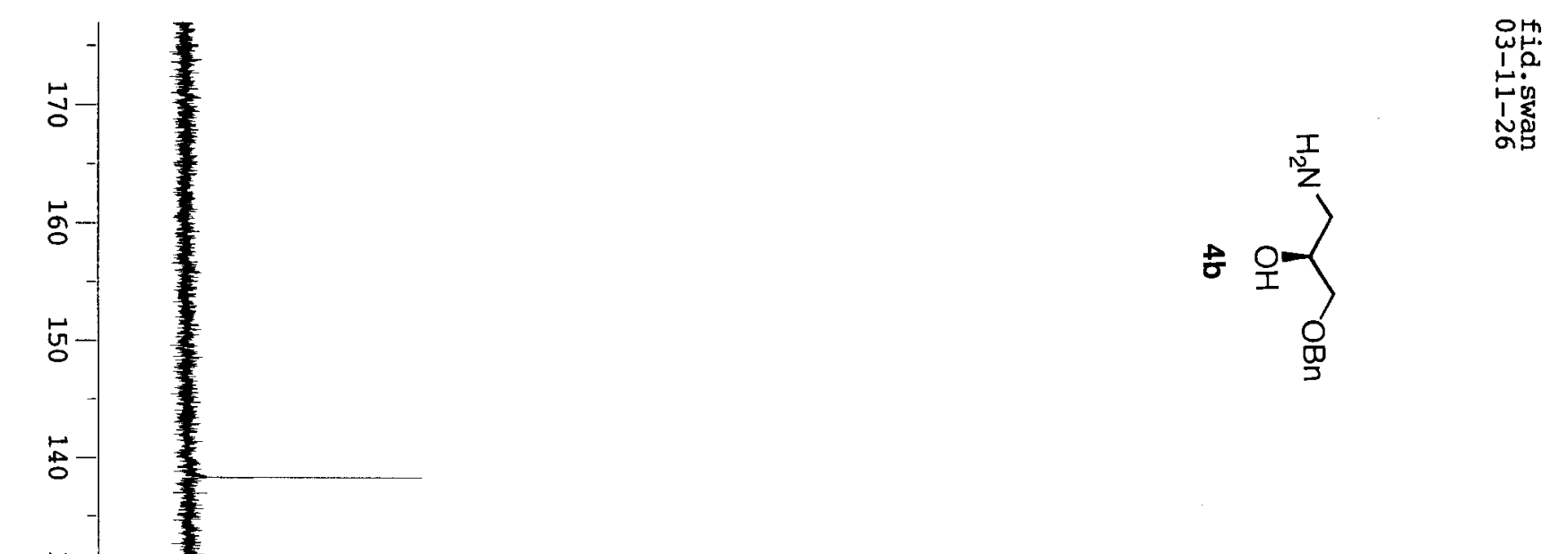

w-

응

占-

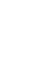

5

๖

\&

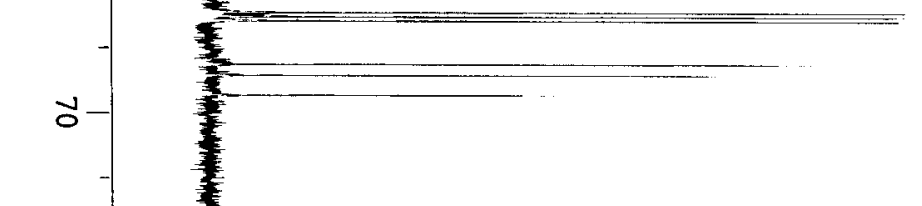

s

v

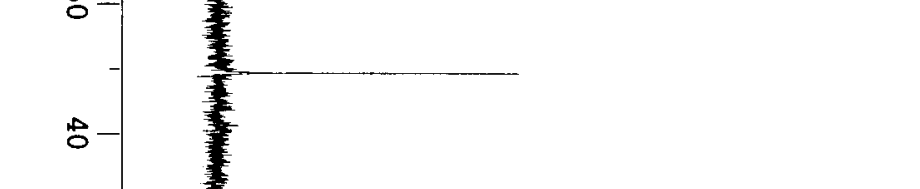

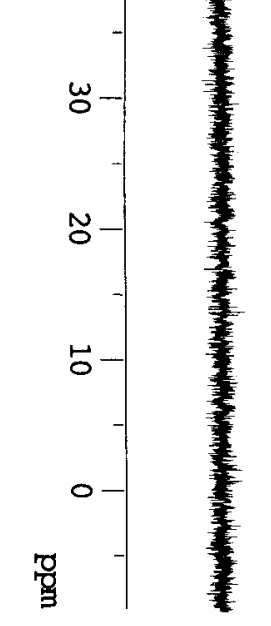



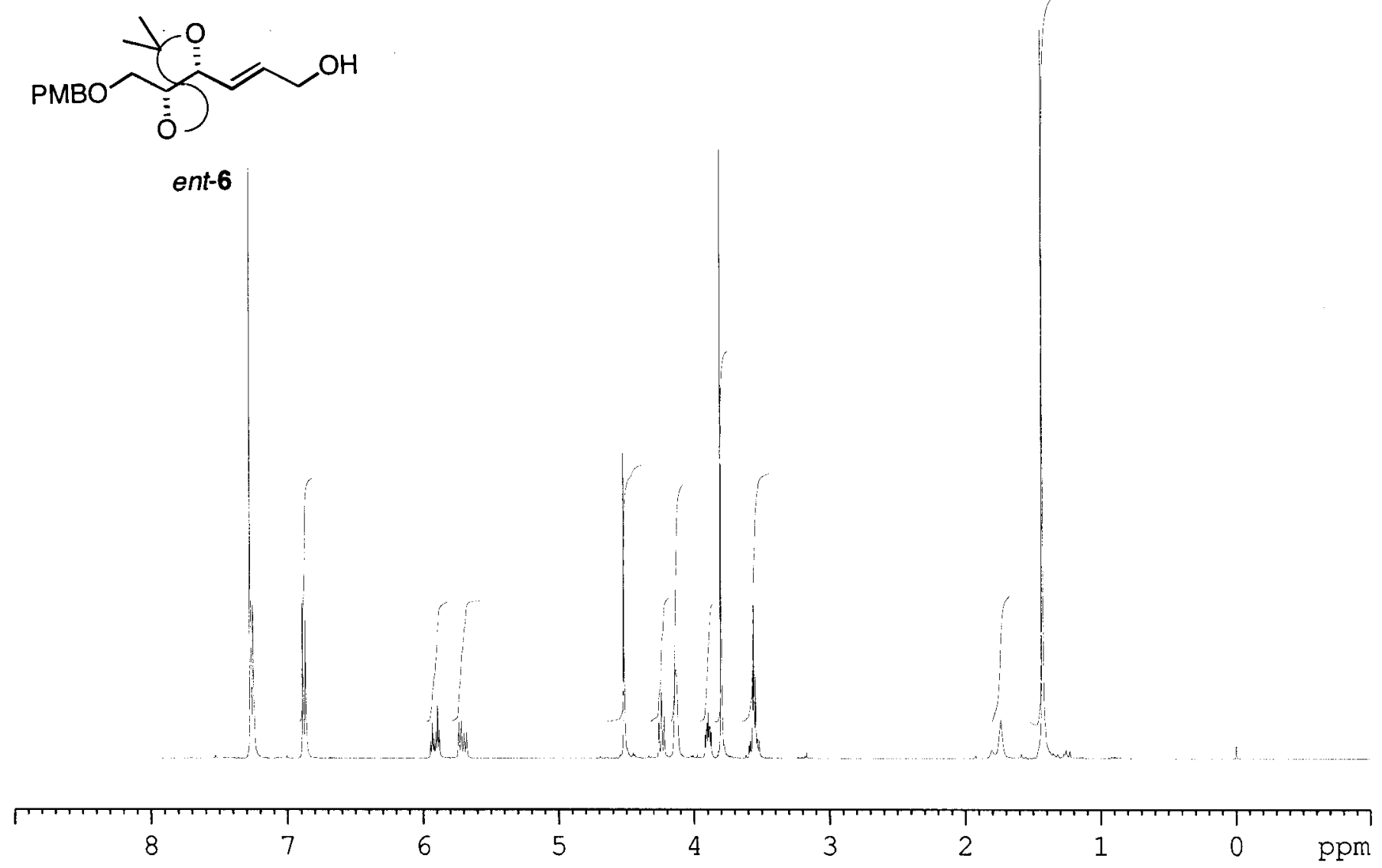

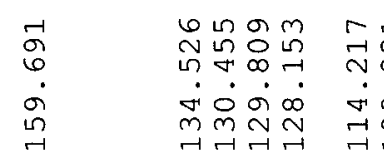

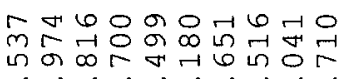

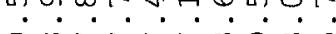

óraraming

\begin{tabular}{l}
$\stackrel{\sigma}{\sim}$ \\
\multirow{2}{*}{}
\end{tabular}

$\left(\begin{array}{ll|l}1 & 1 & \\ & 1 & 1\end{array}\right.$

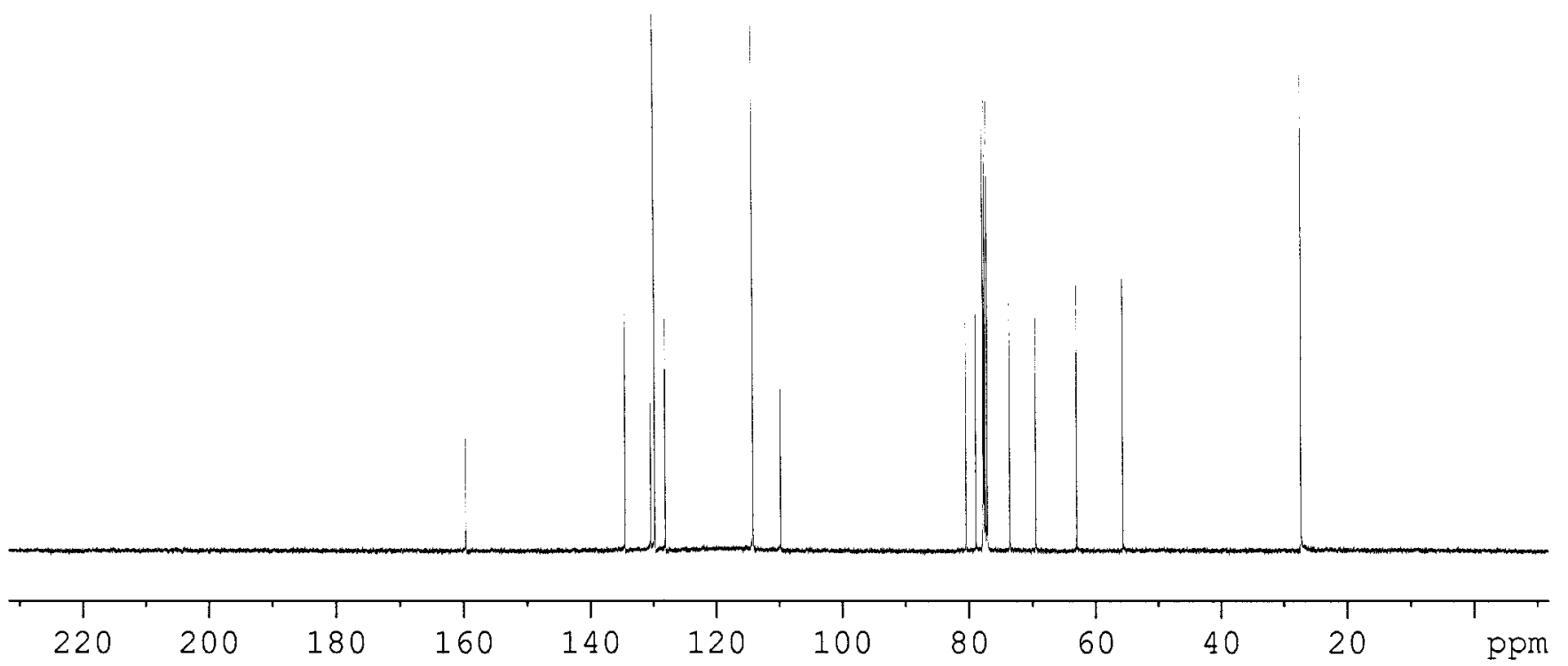




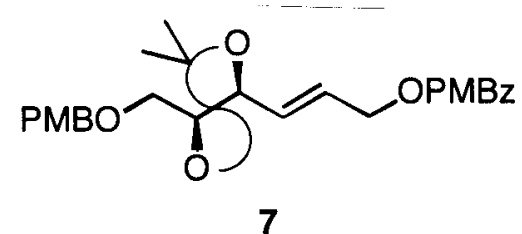

7

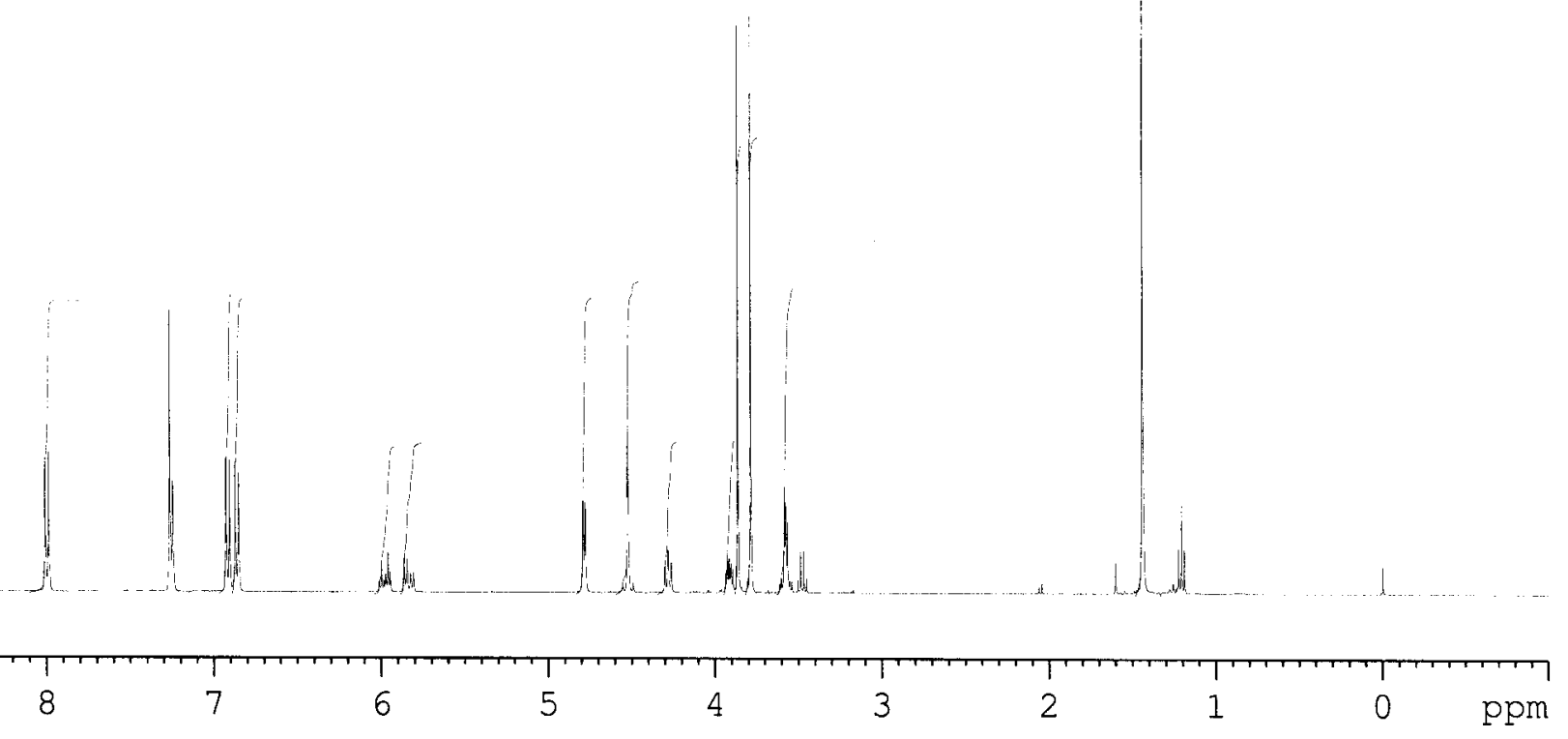

\begin{tabular}{|c|c|c|c|c|}
\hline$m \circ \mathrm{m}$ & $\infty m \curvearrowleft \infty r \nabla \backsim \omega \infty$ & & & \\
\hline $\begin{array}{ll}N \infty & \infty \\
m \infty & \infty\end{array}$ & 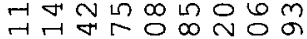 & 댕ㄷㅇㅇㅢ & $m$ & $m$ \\
\hline$\dot{\omega} \dot{\circ}$ & $\dot{\sim} \dot{0} \dot{\circ} \dot{\sigma} \dot{\sim} \dot{\nabla} \dot{\sigma}$ & 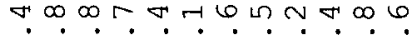 & 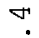 & $r$ \\
\hline 6010 & $m m M N N H O$ & $0 \infty$ r R & $\Gamma$ & n \\
\hline & 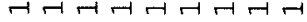 & orererth & $\sim$ & -1 \\
\hline
\end{tabular}

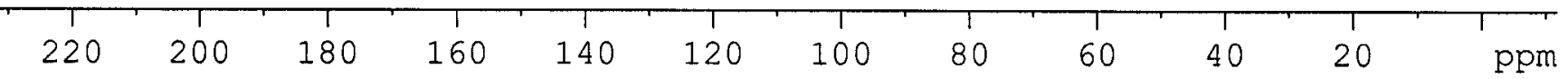



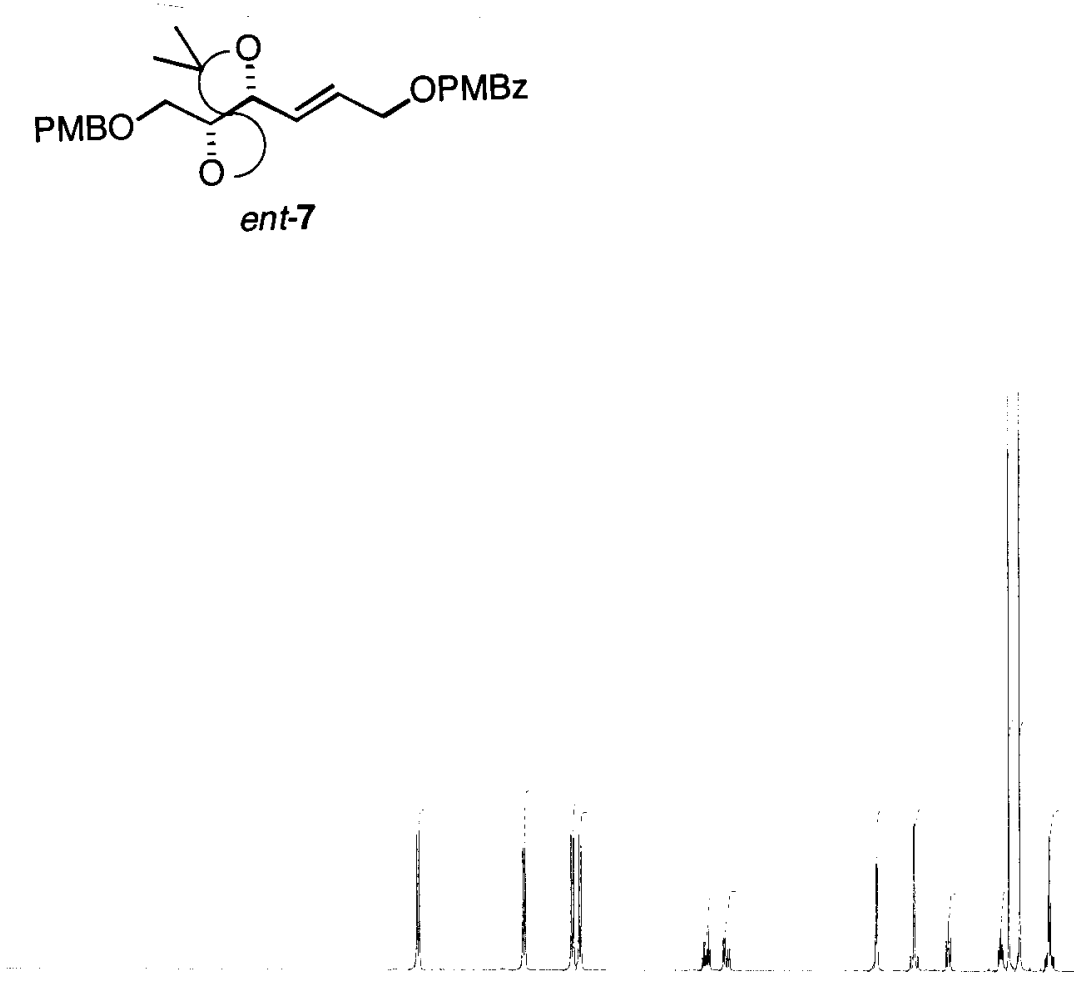

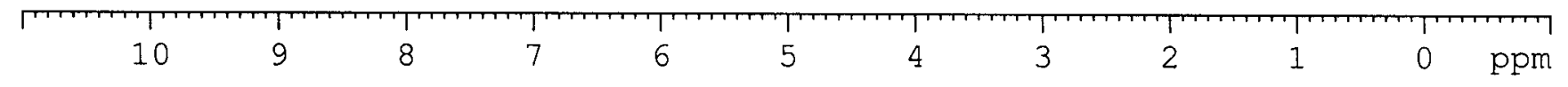

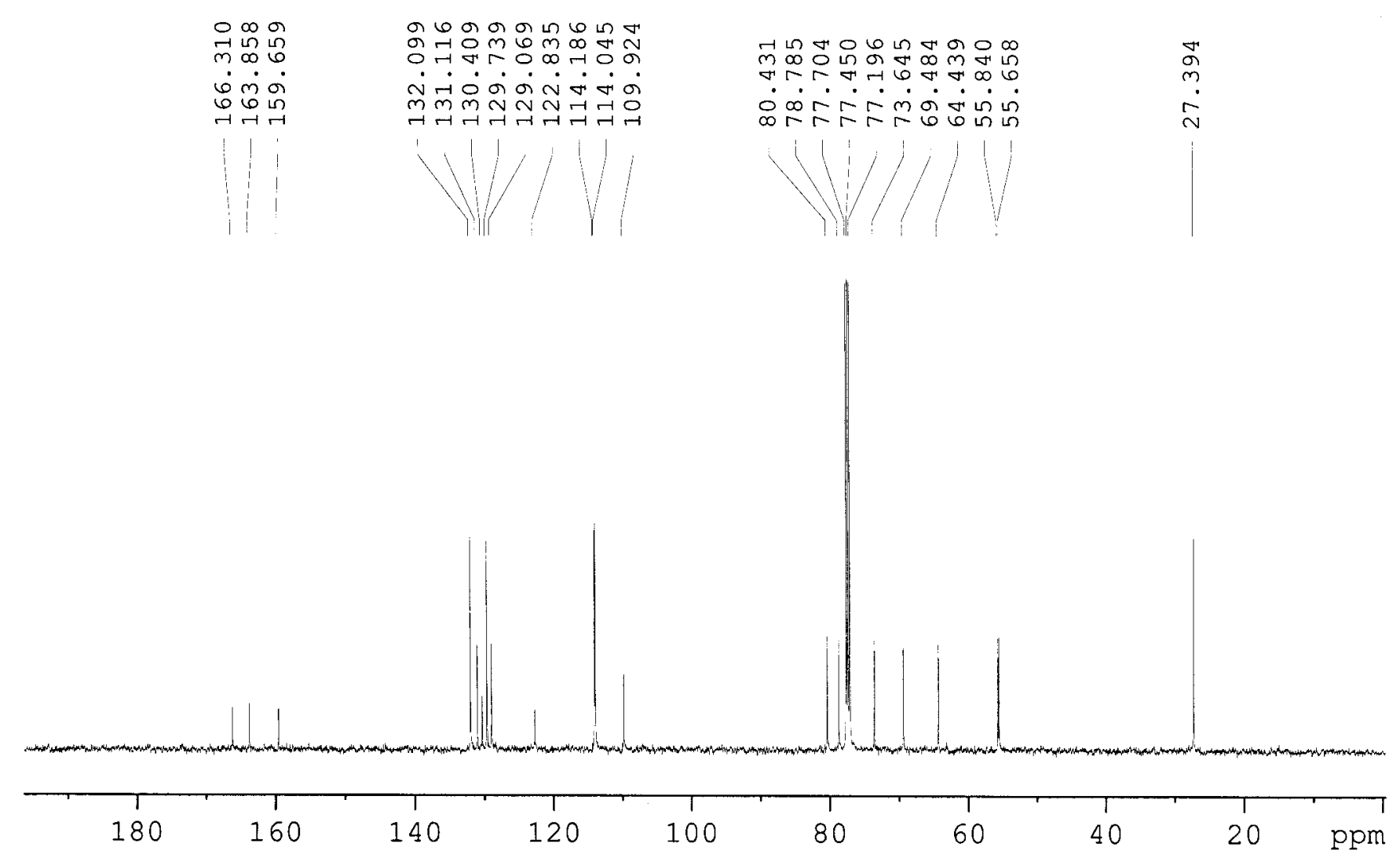



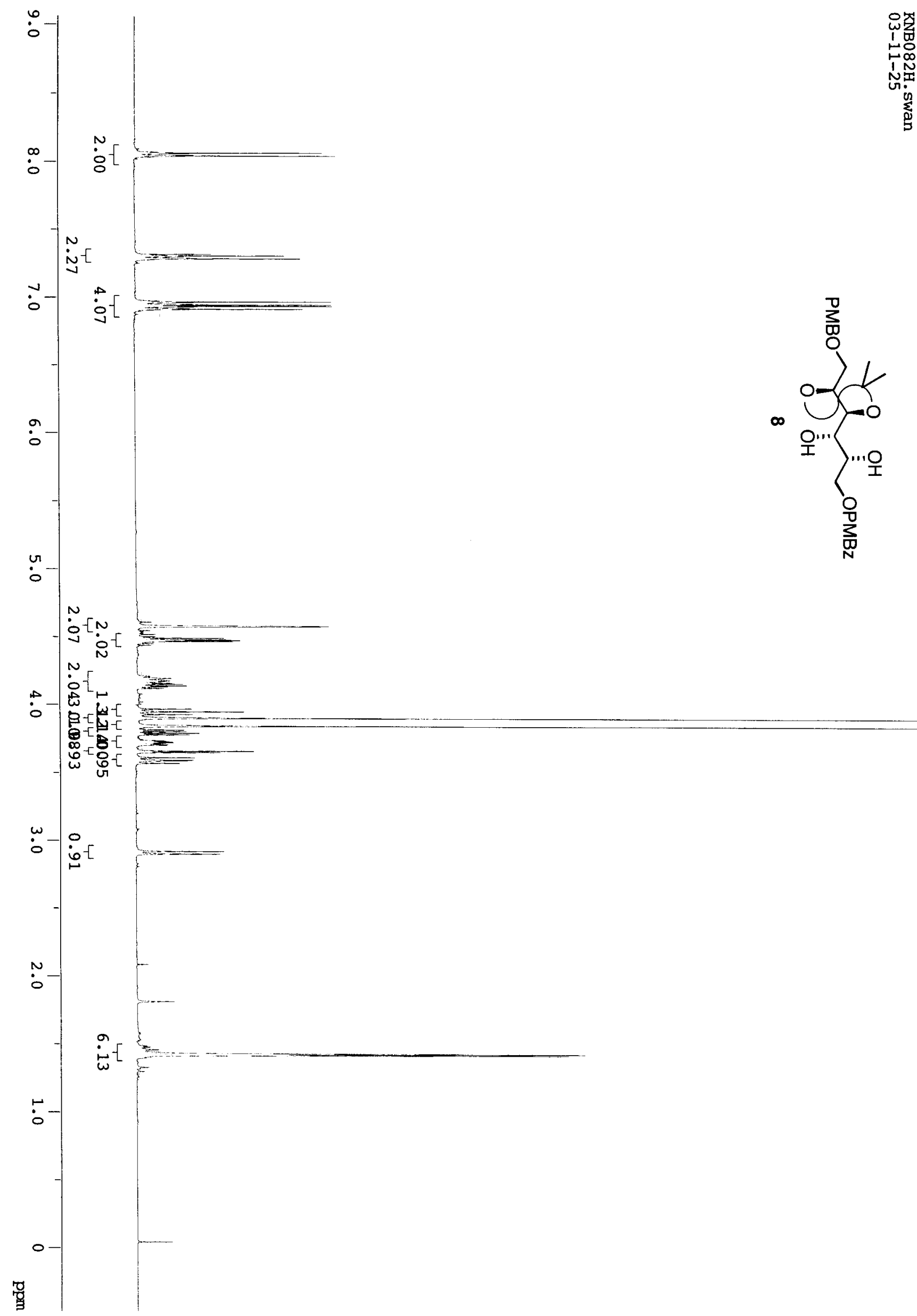


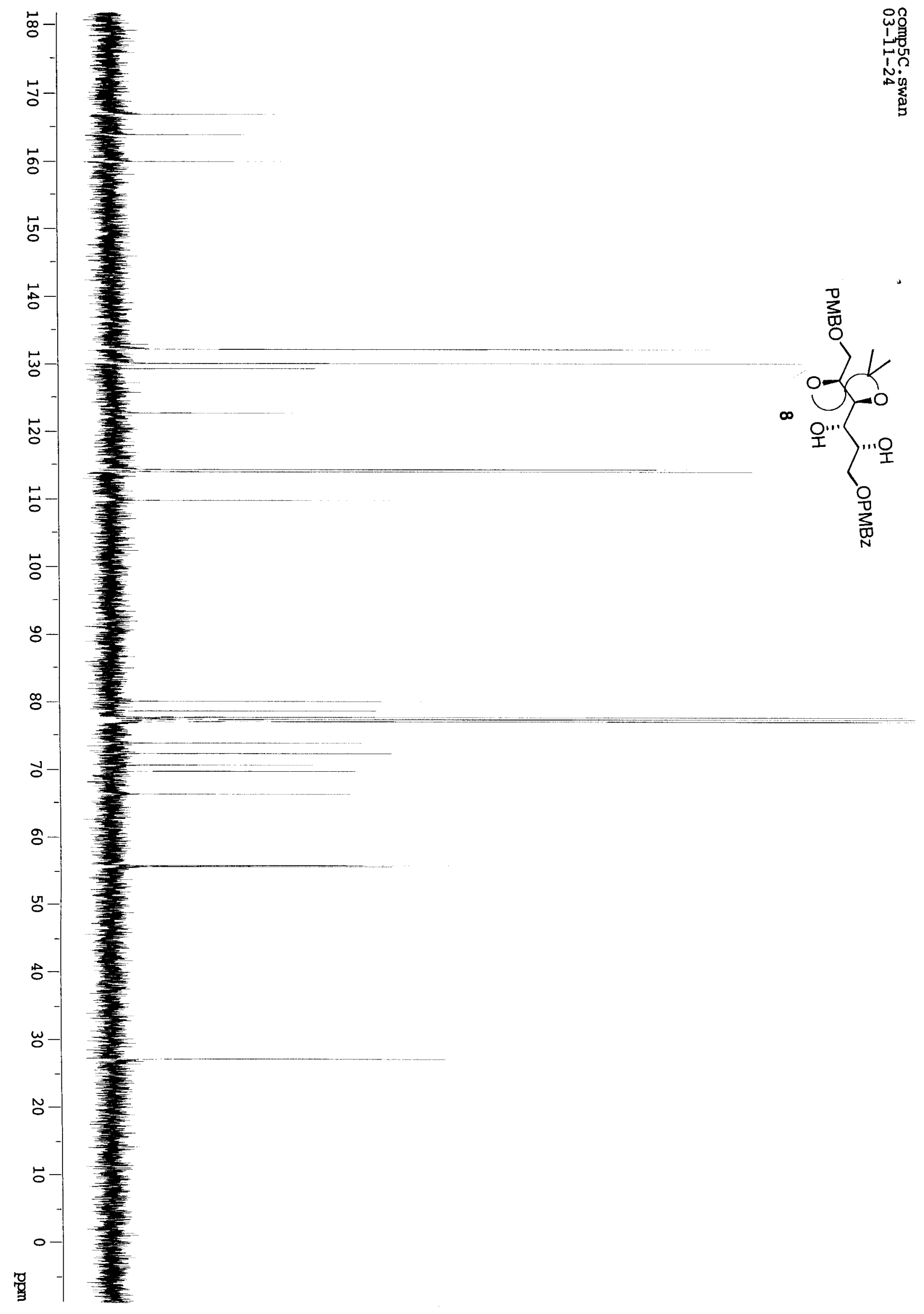



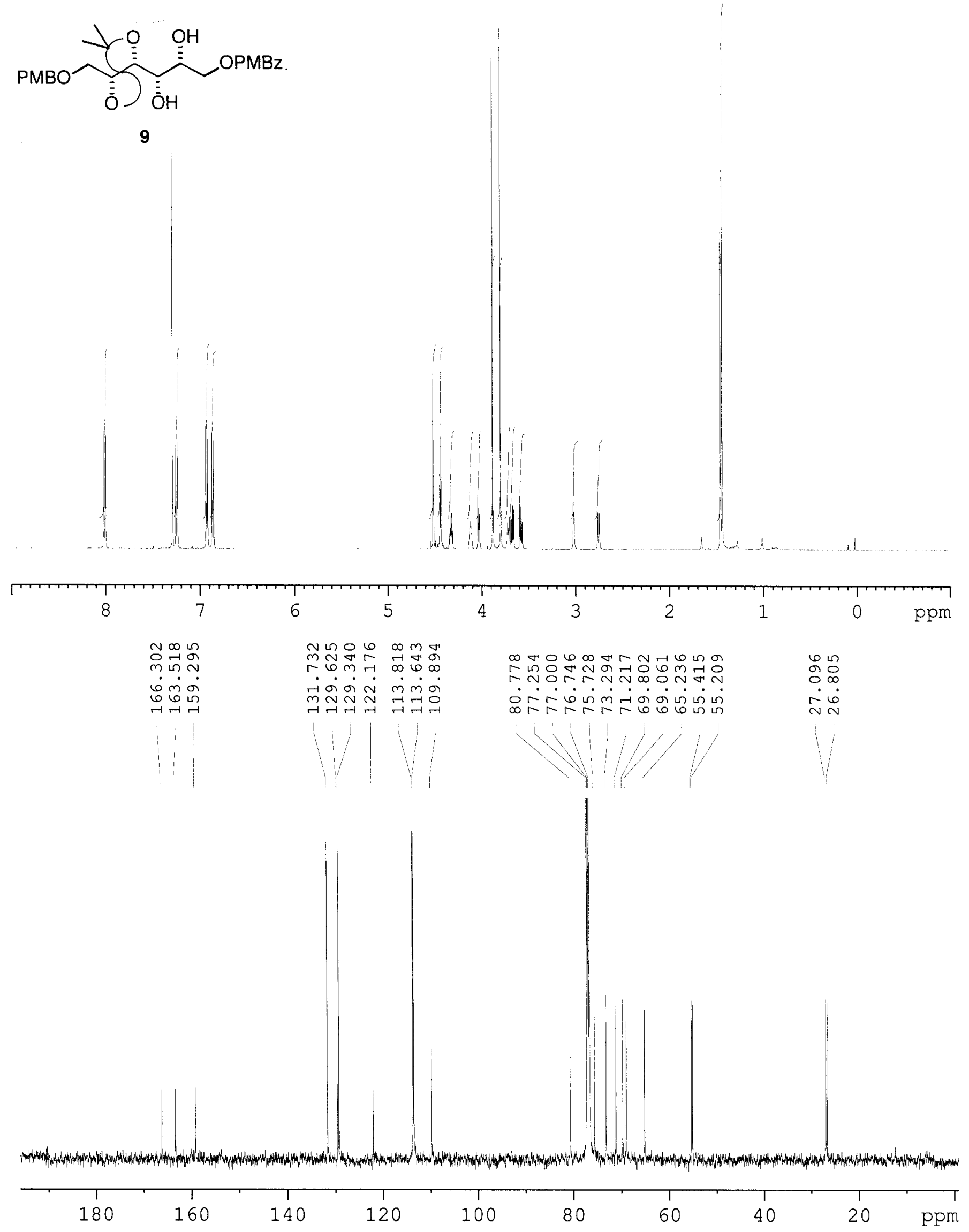
$\overbrace{O}^{2}$

10
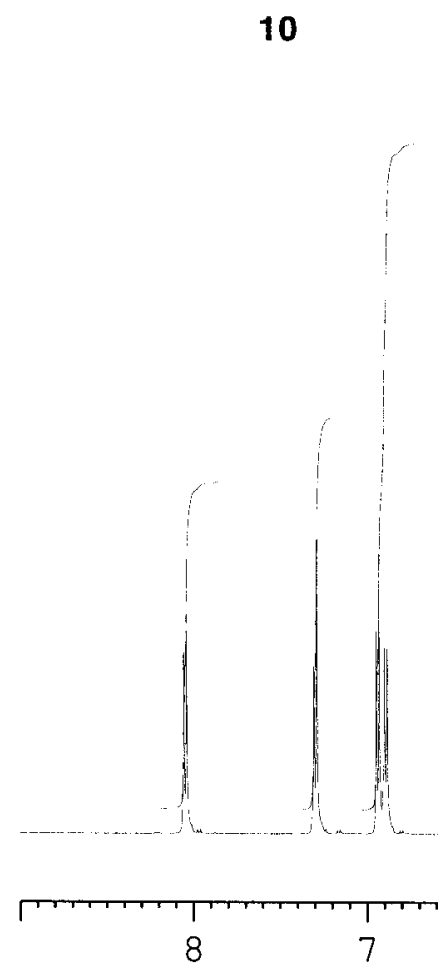

8

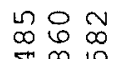

ம்

$\begin{array}{lll}0 & 0 & 0 \\ 1 & -1\end{array}$

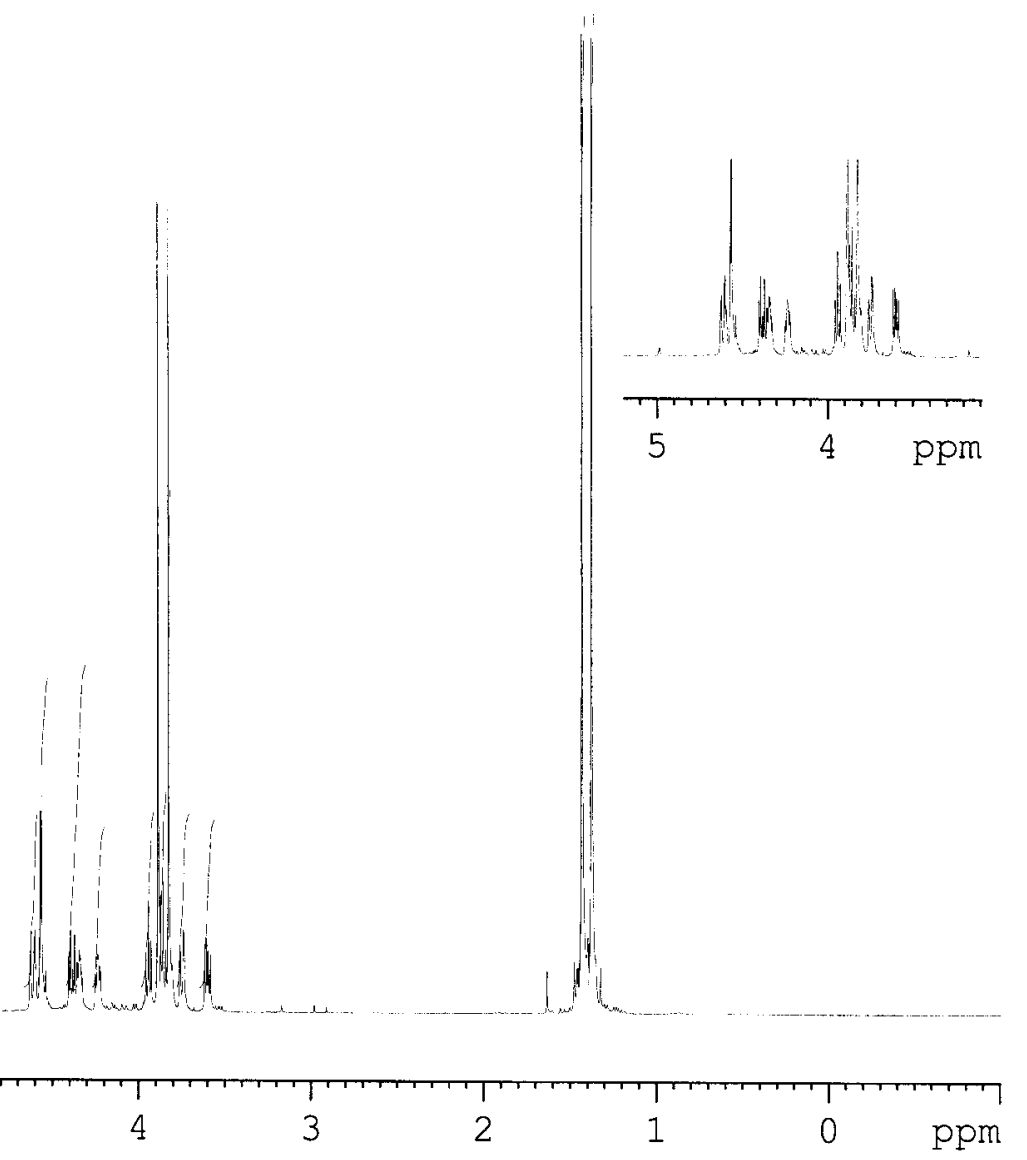

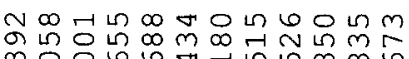

. . . . .

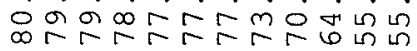

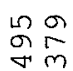

ํ․․

iㅗ

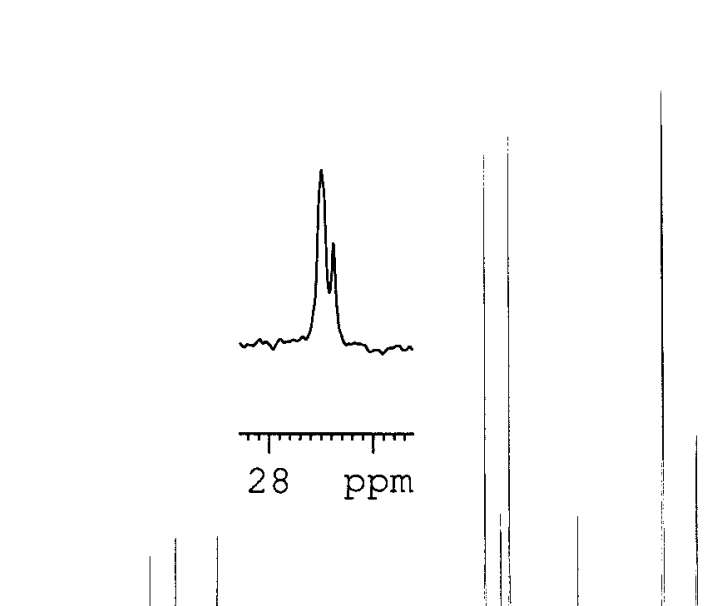

y.
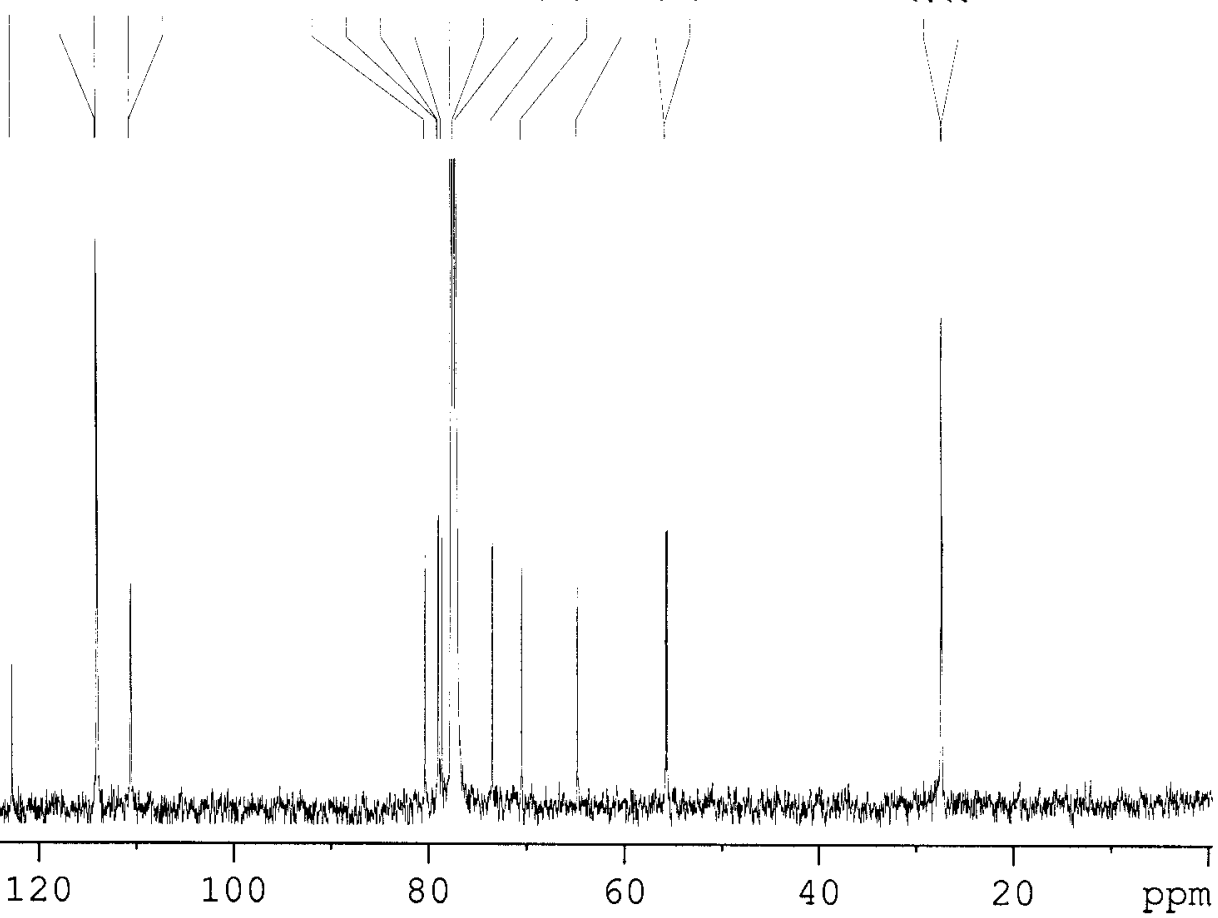

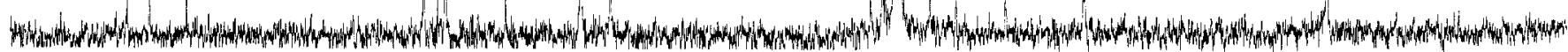


PMBO

11
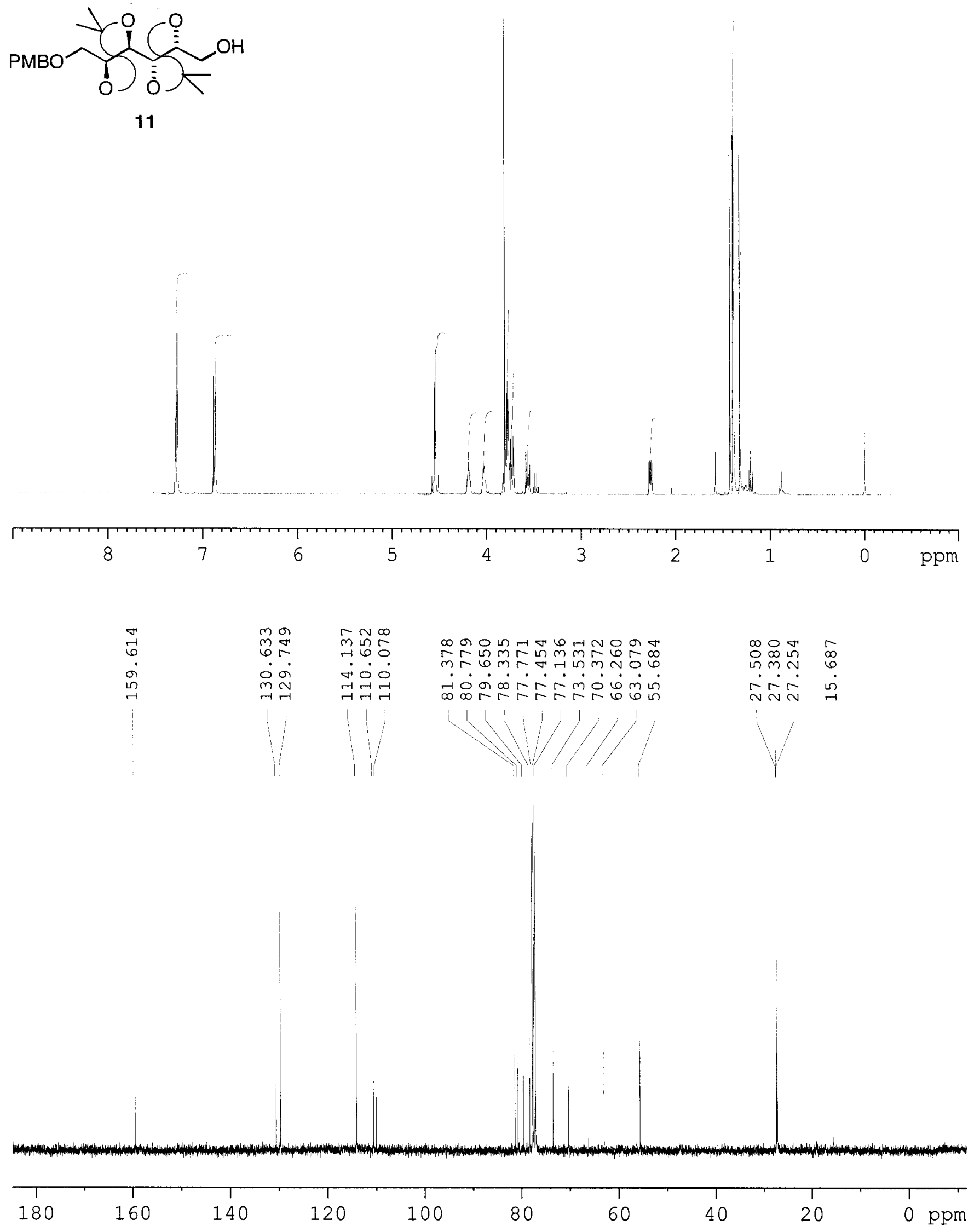


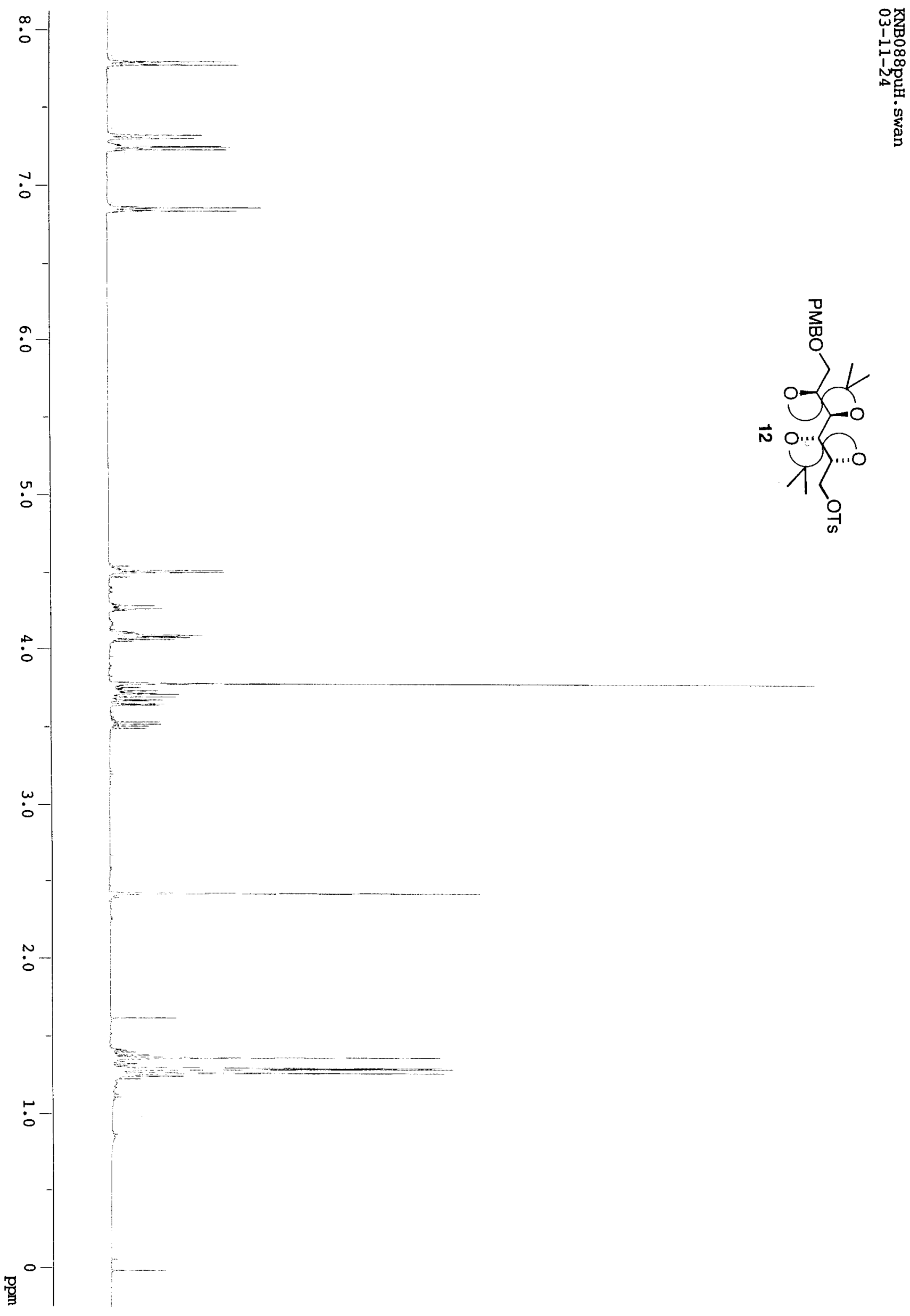




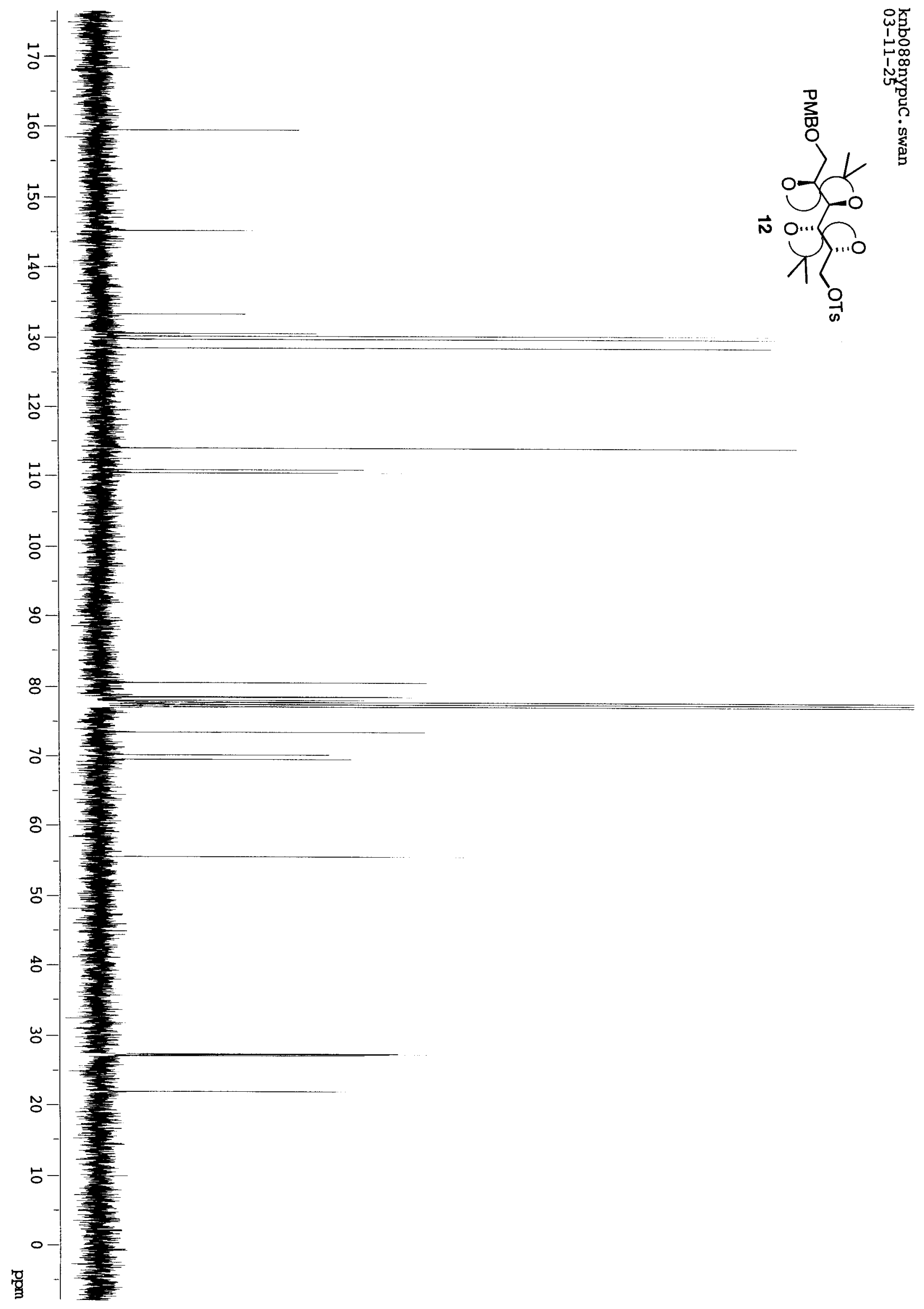




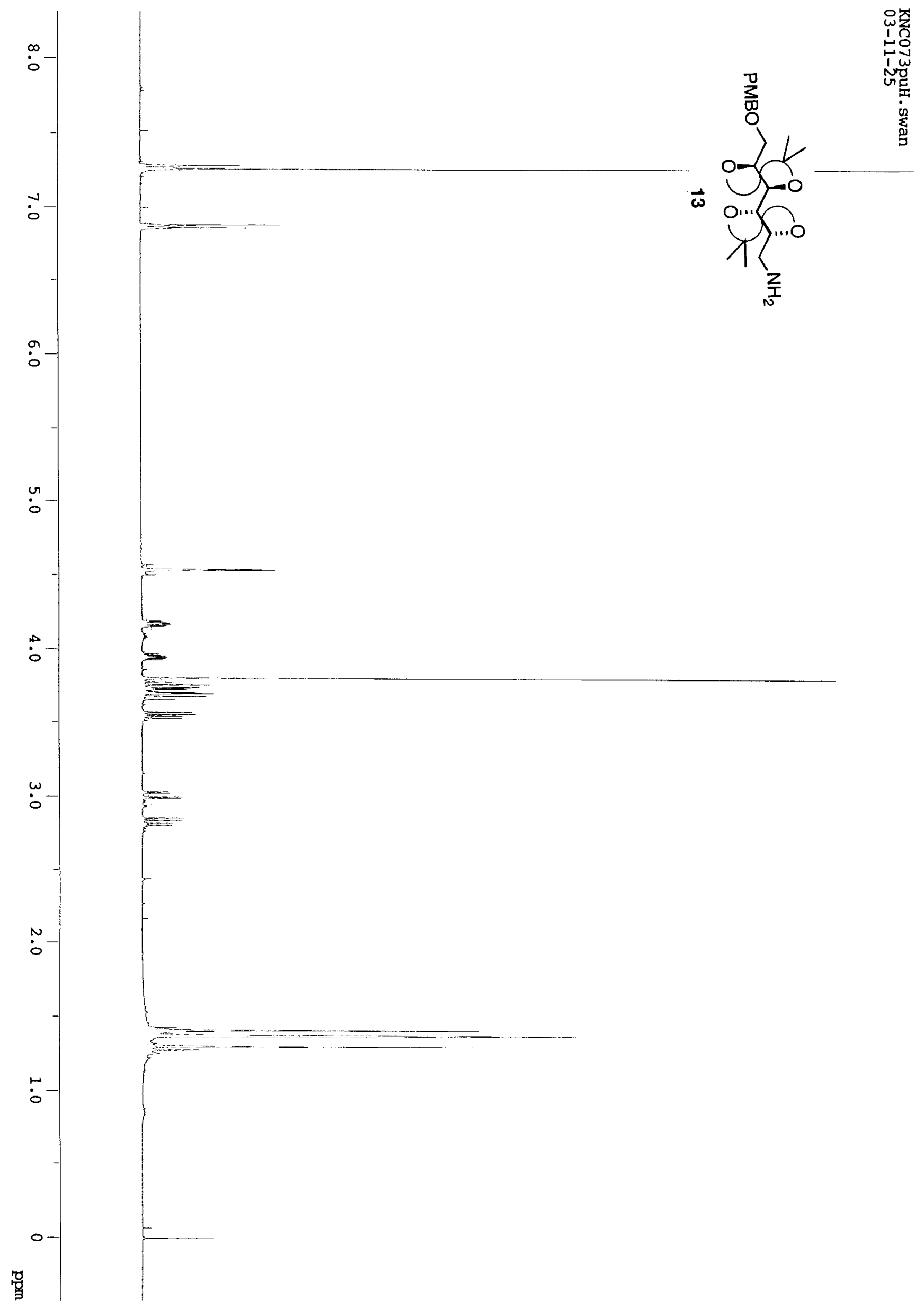




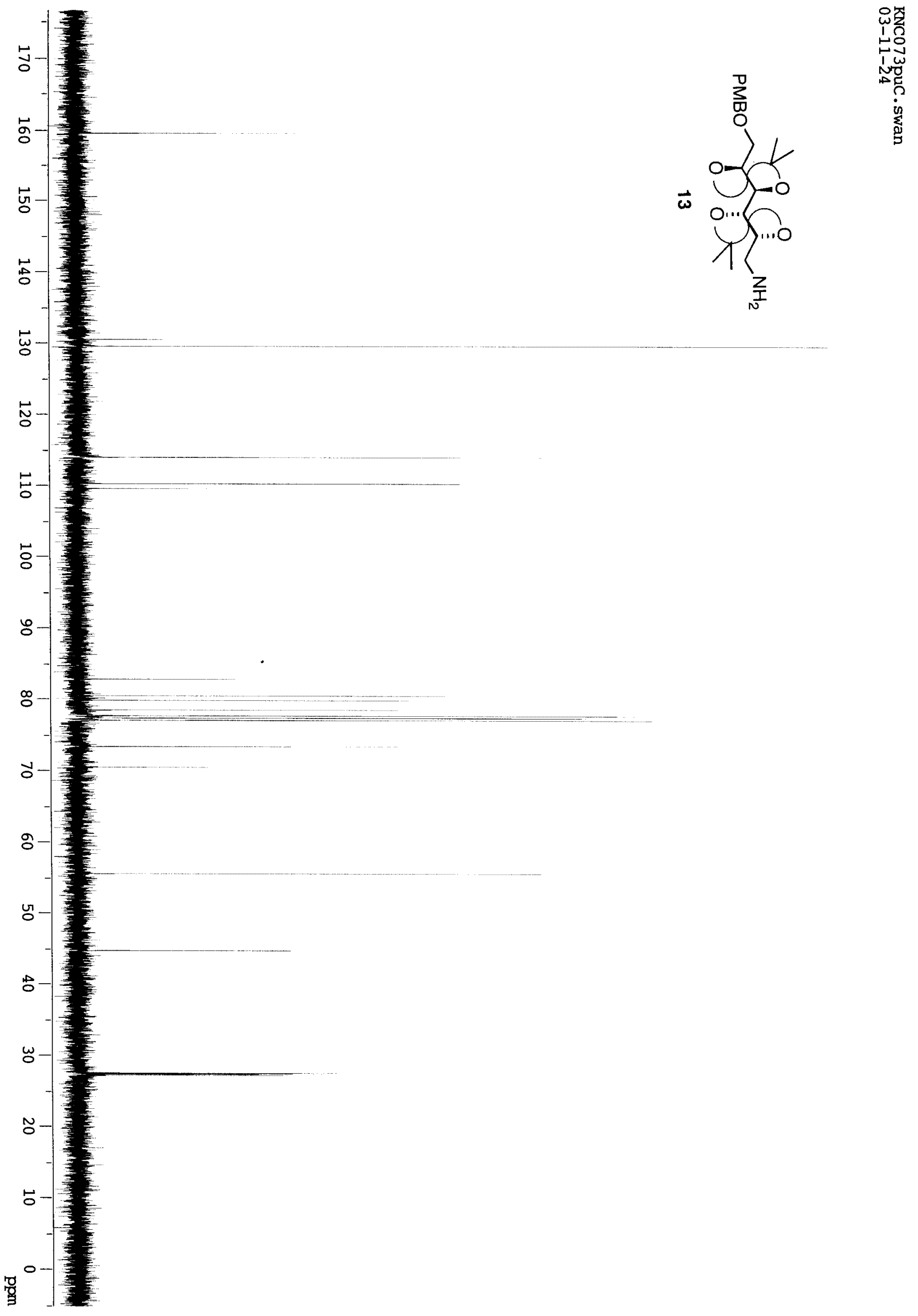




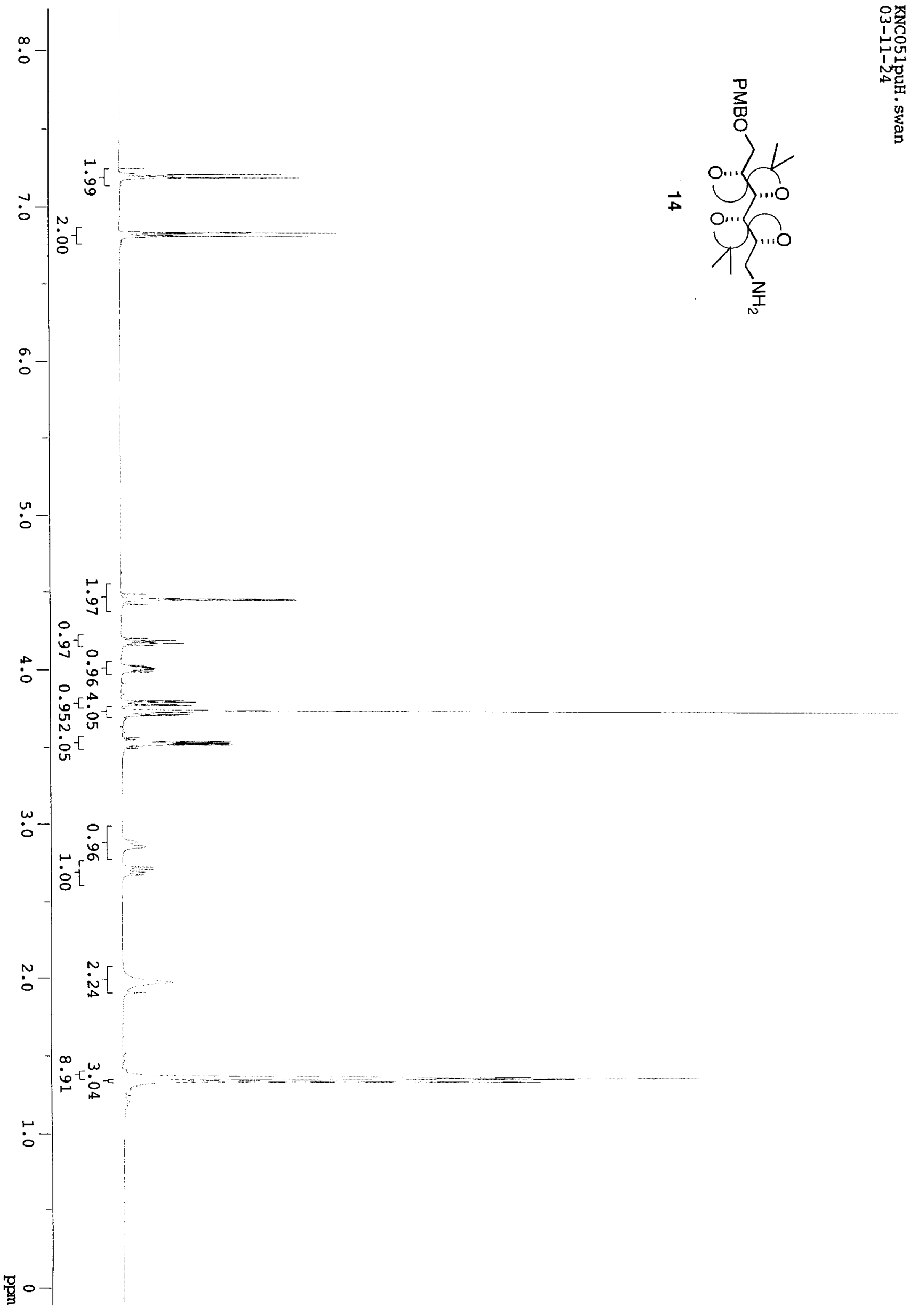




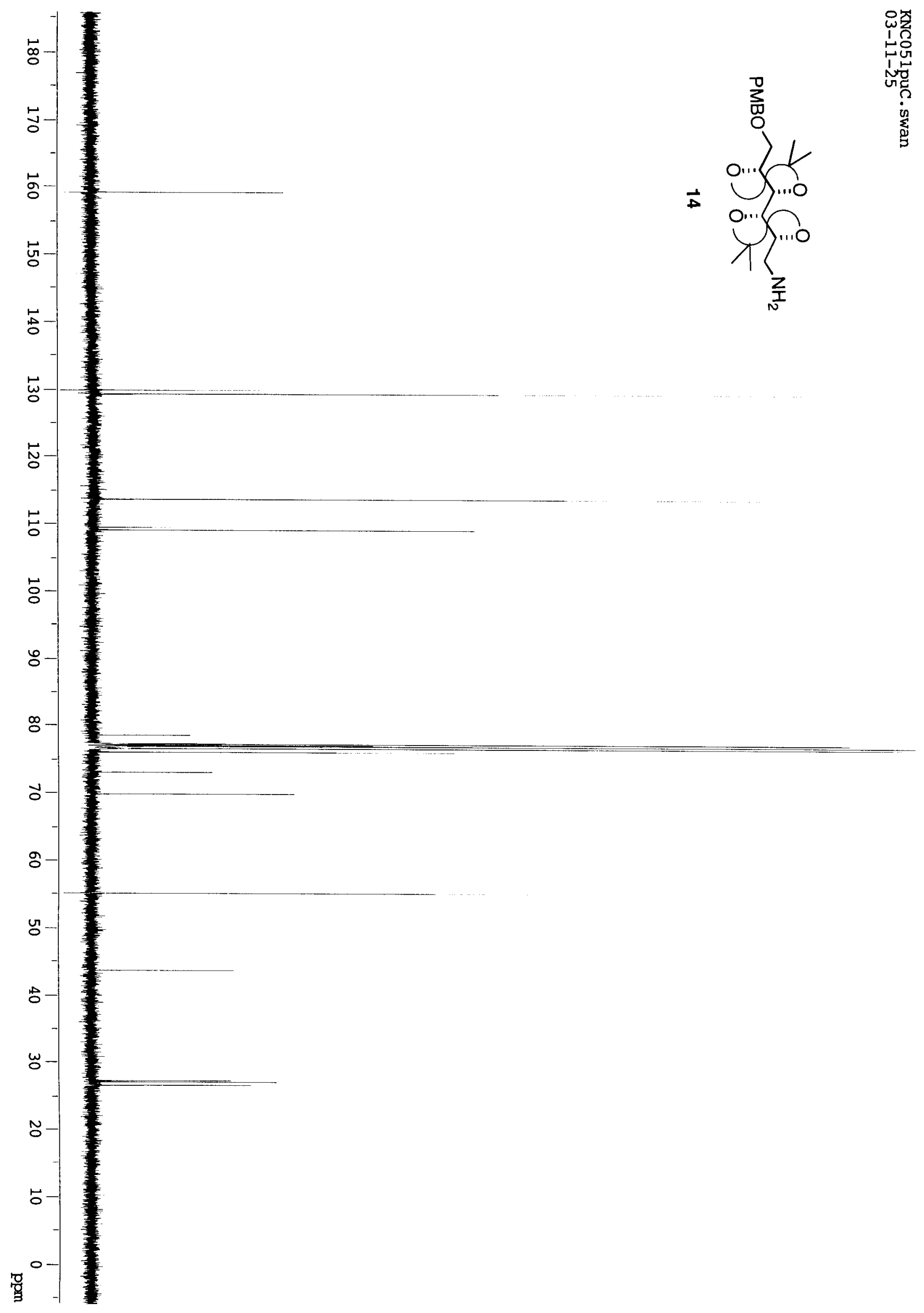




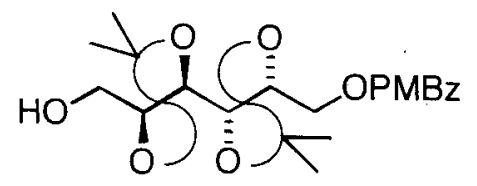

15
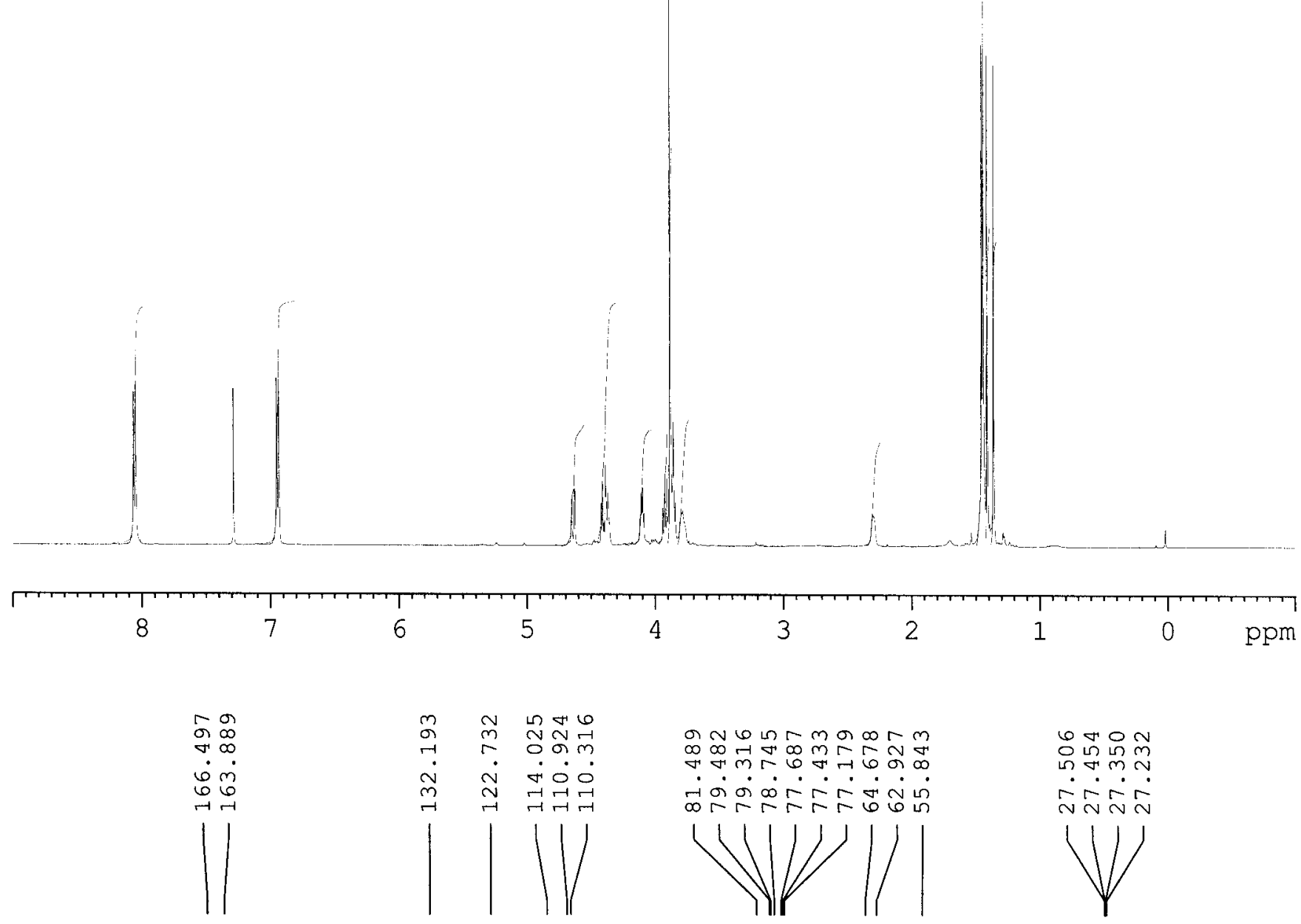

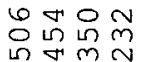

iris

(V)

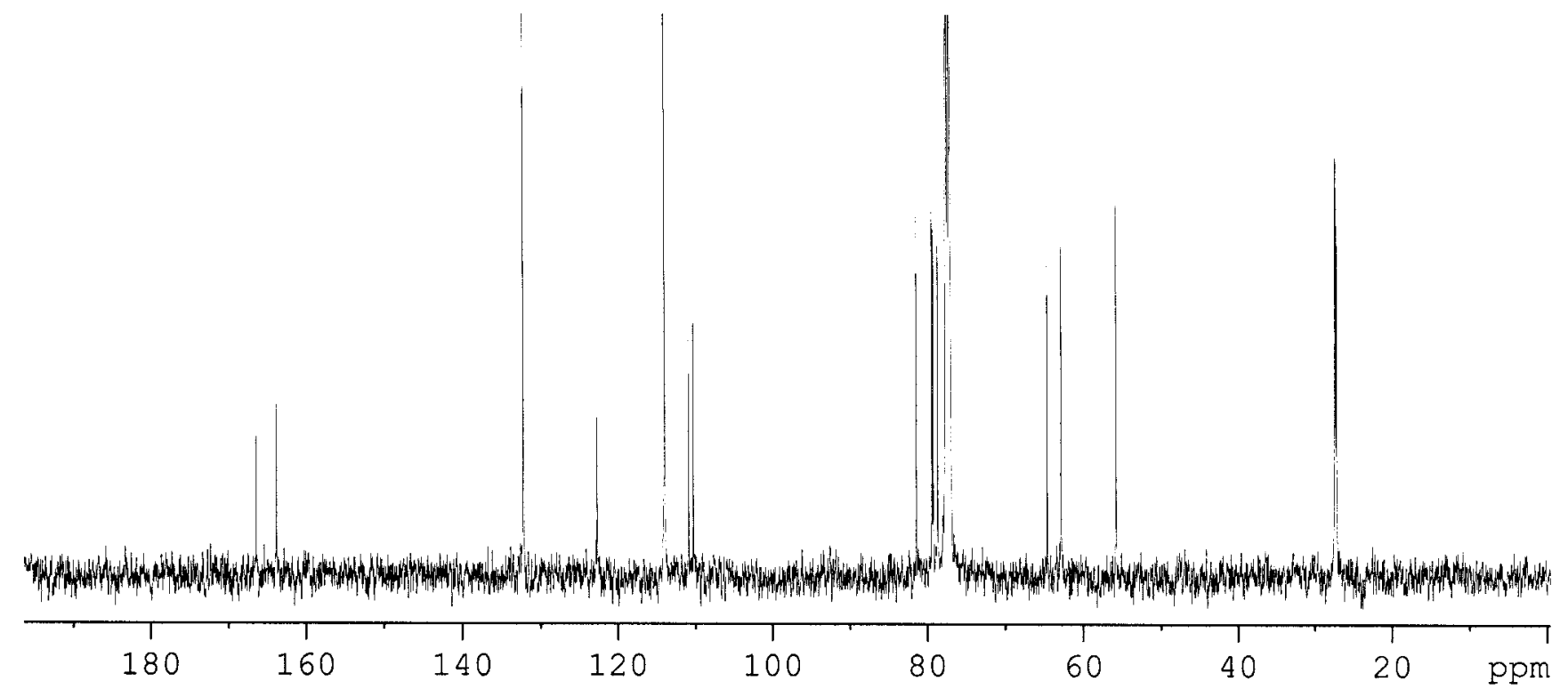




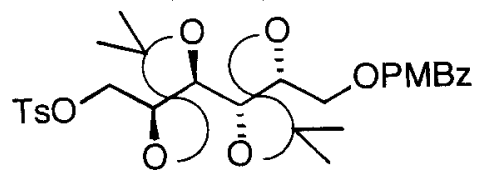

16

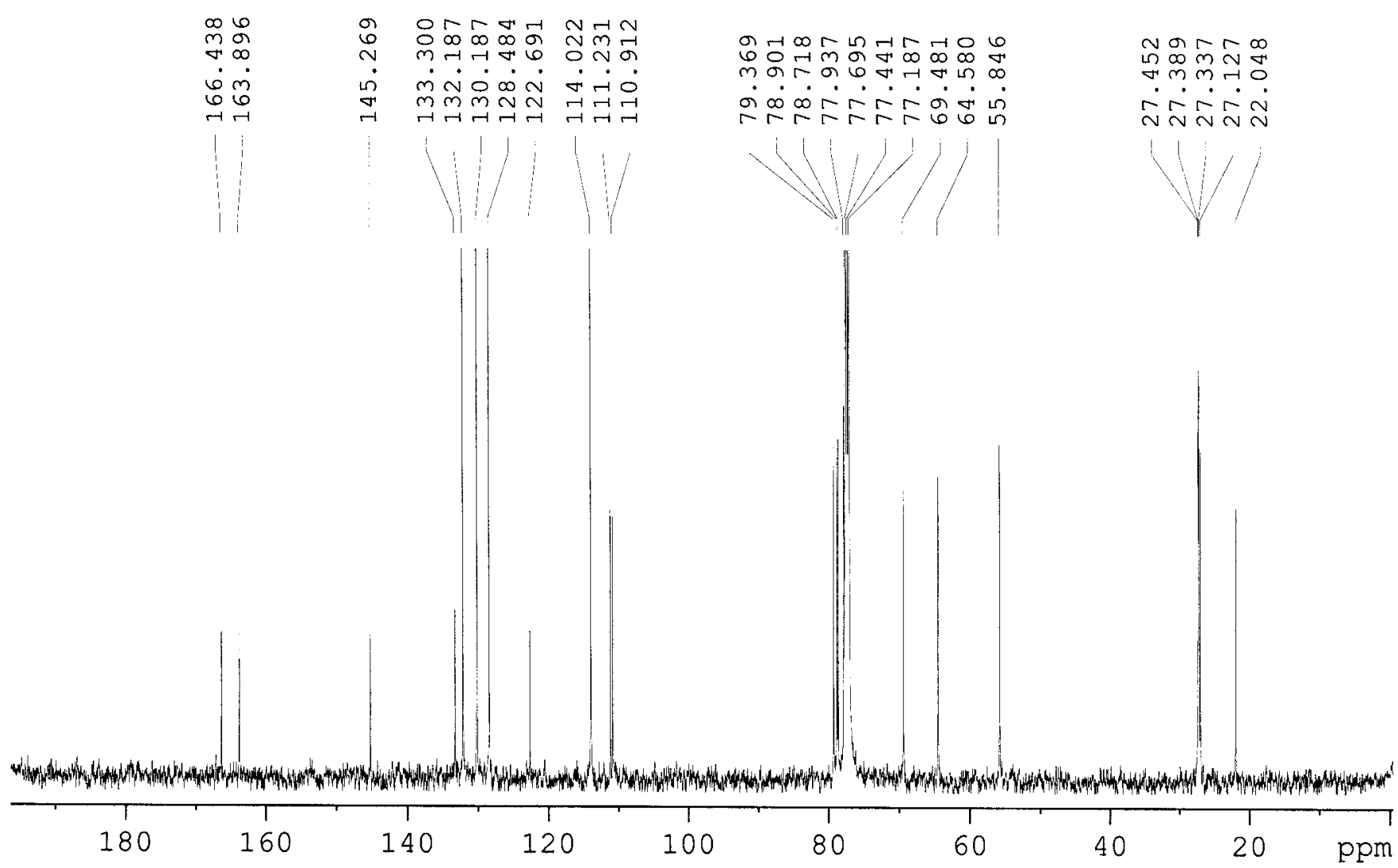




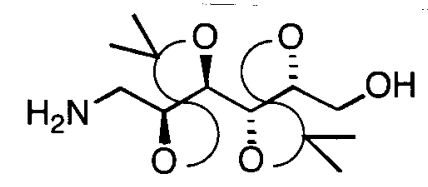

17
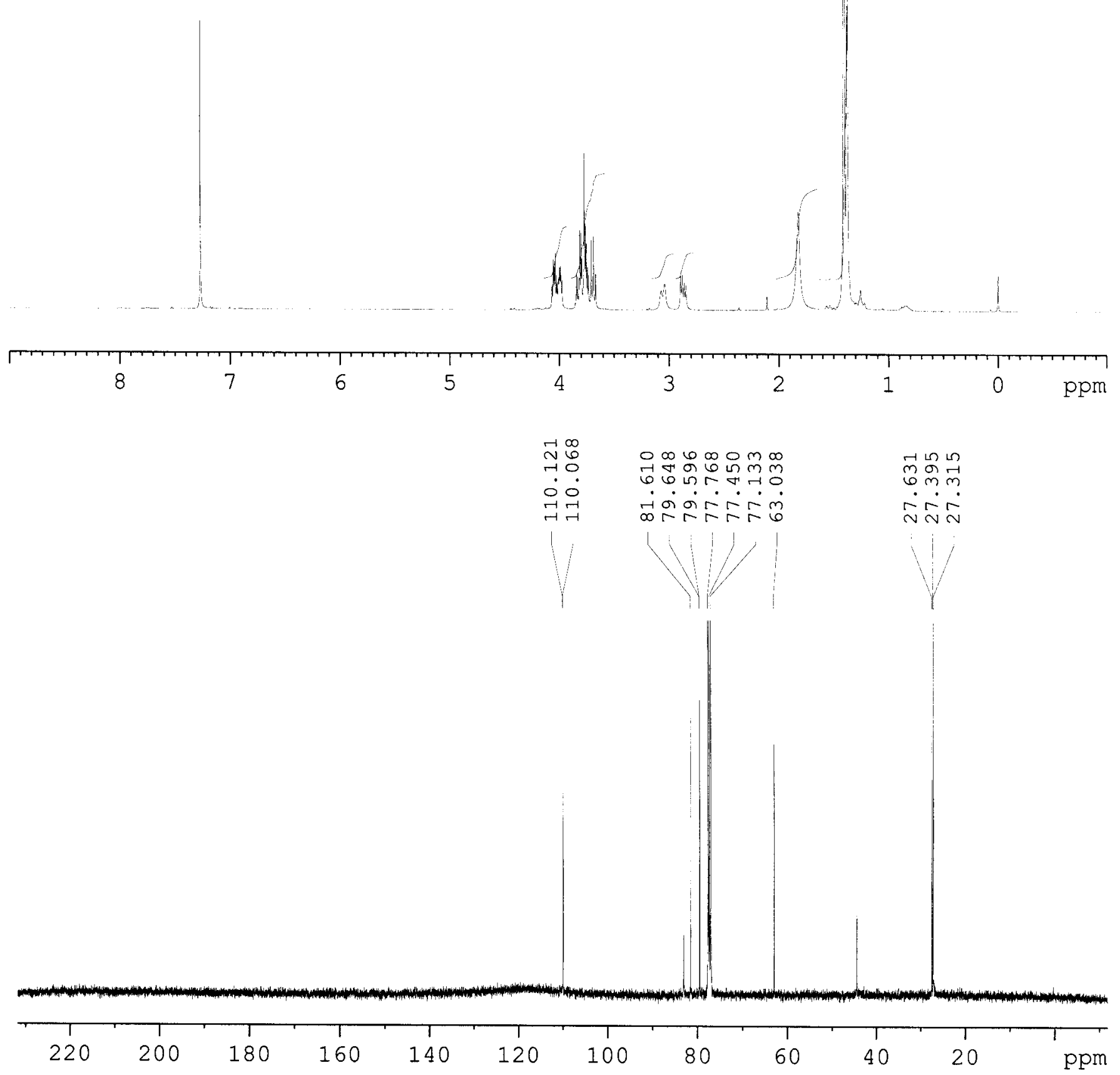


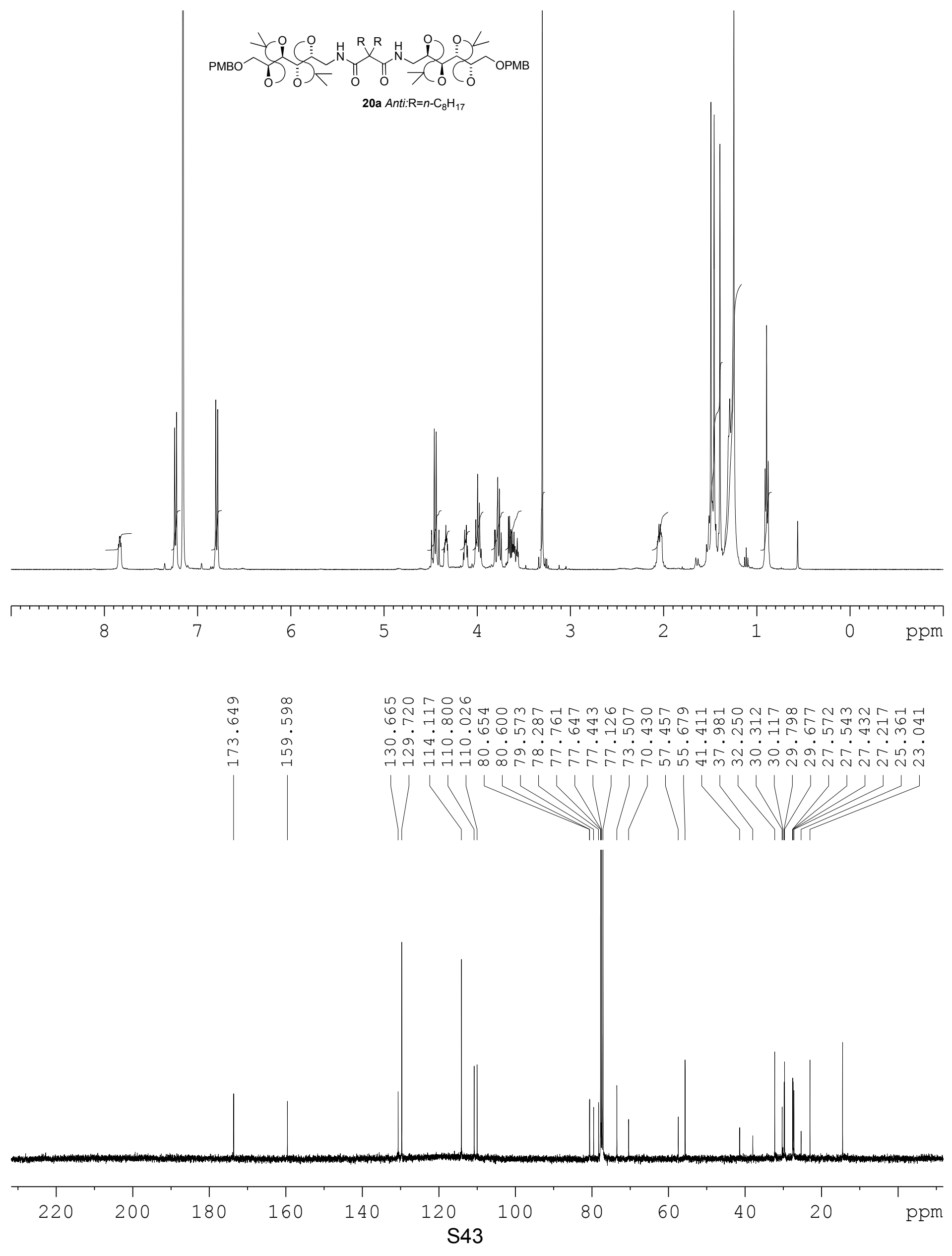




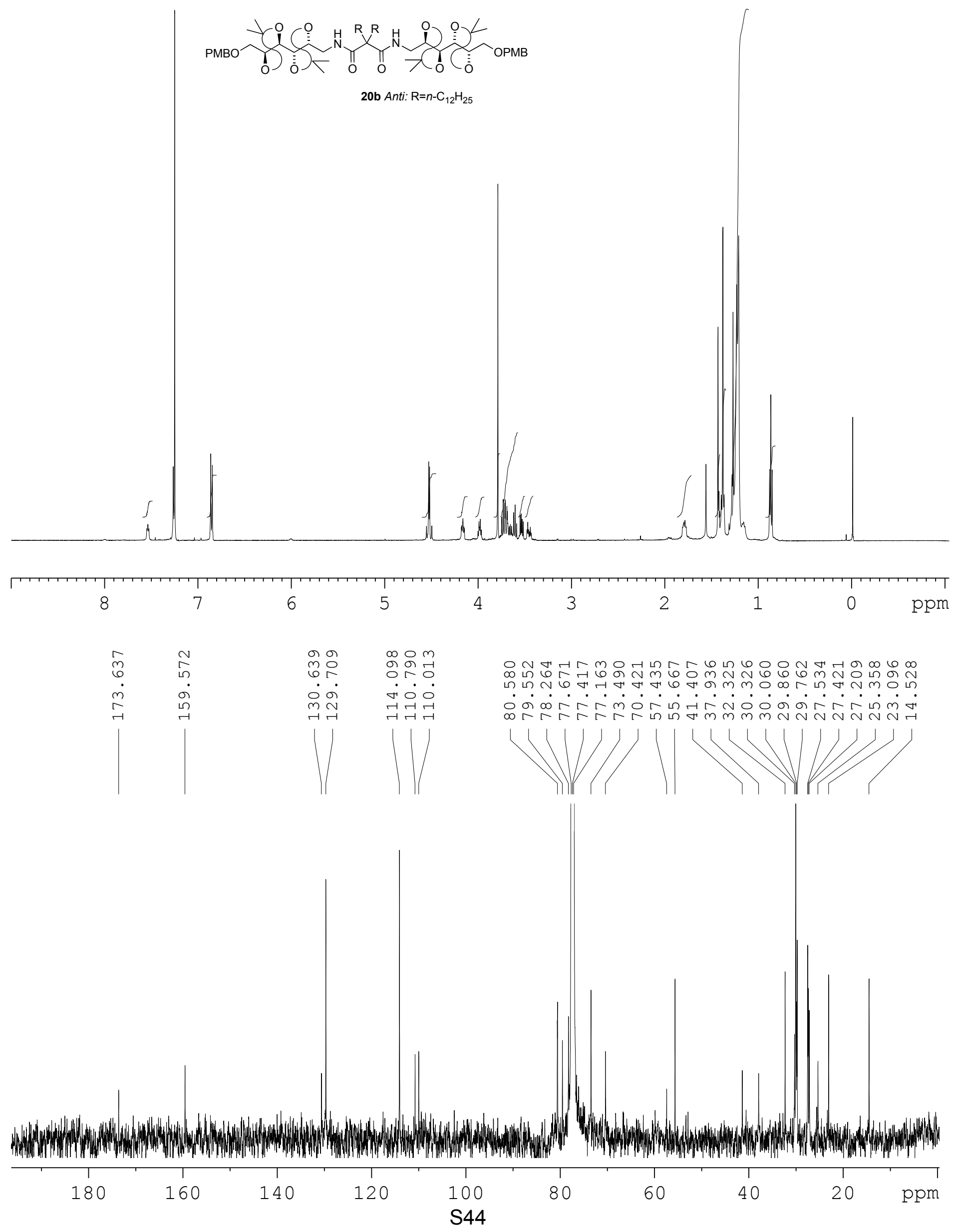




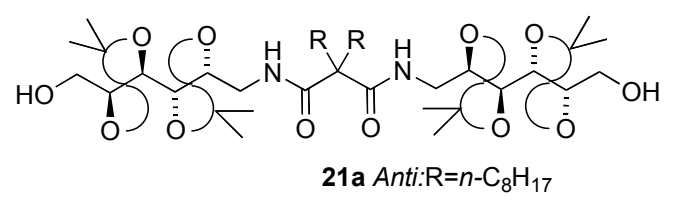




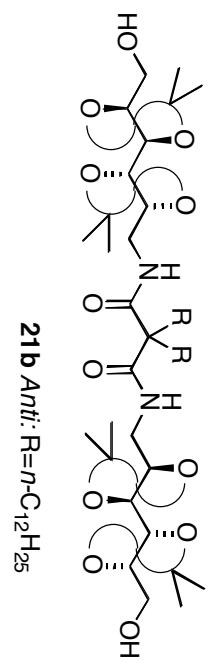




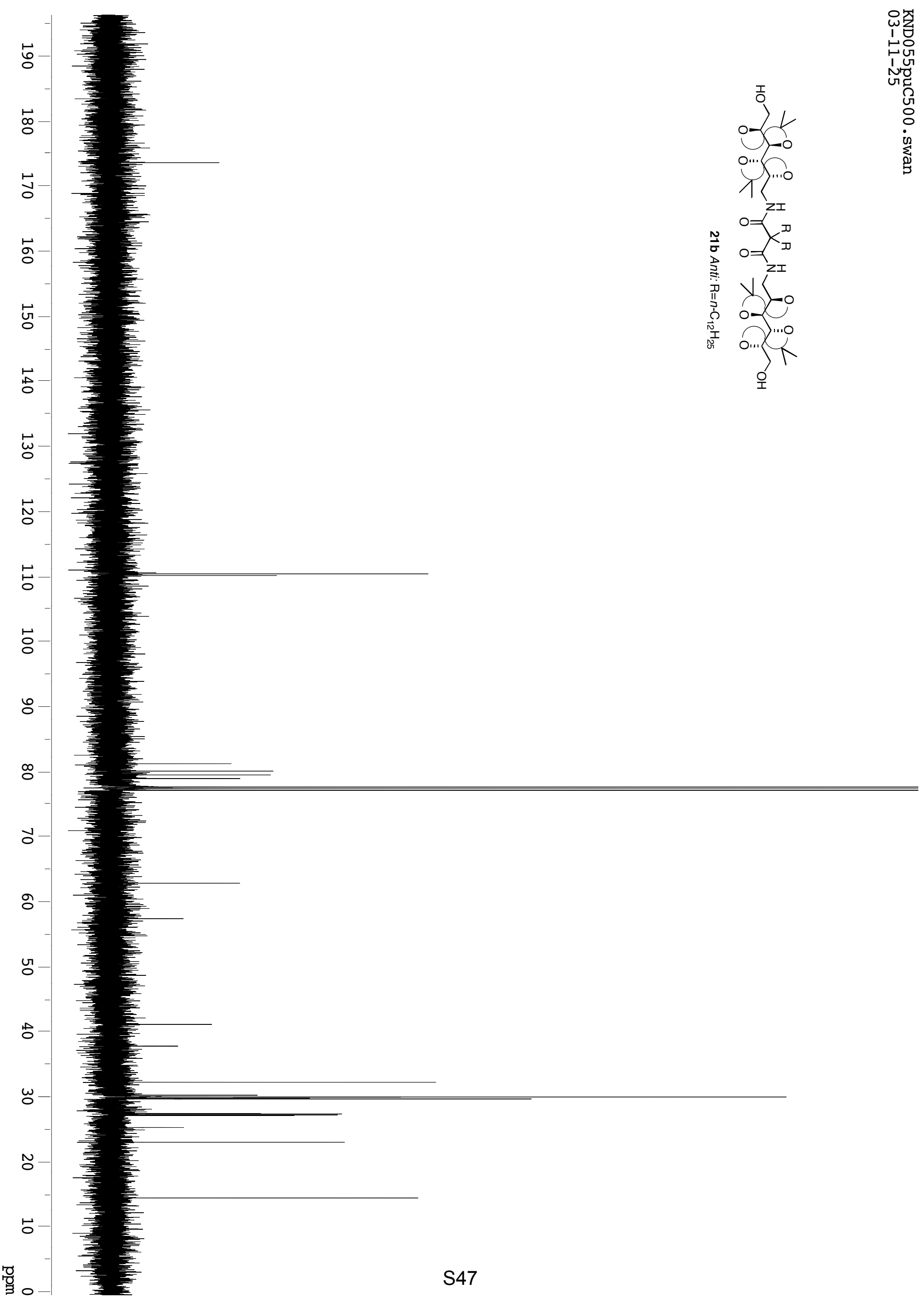



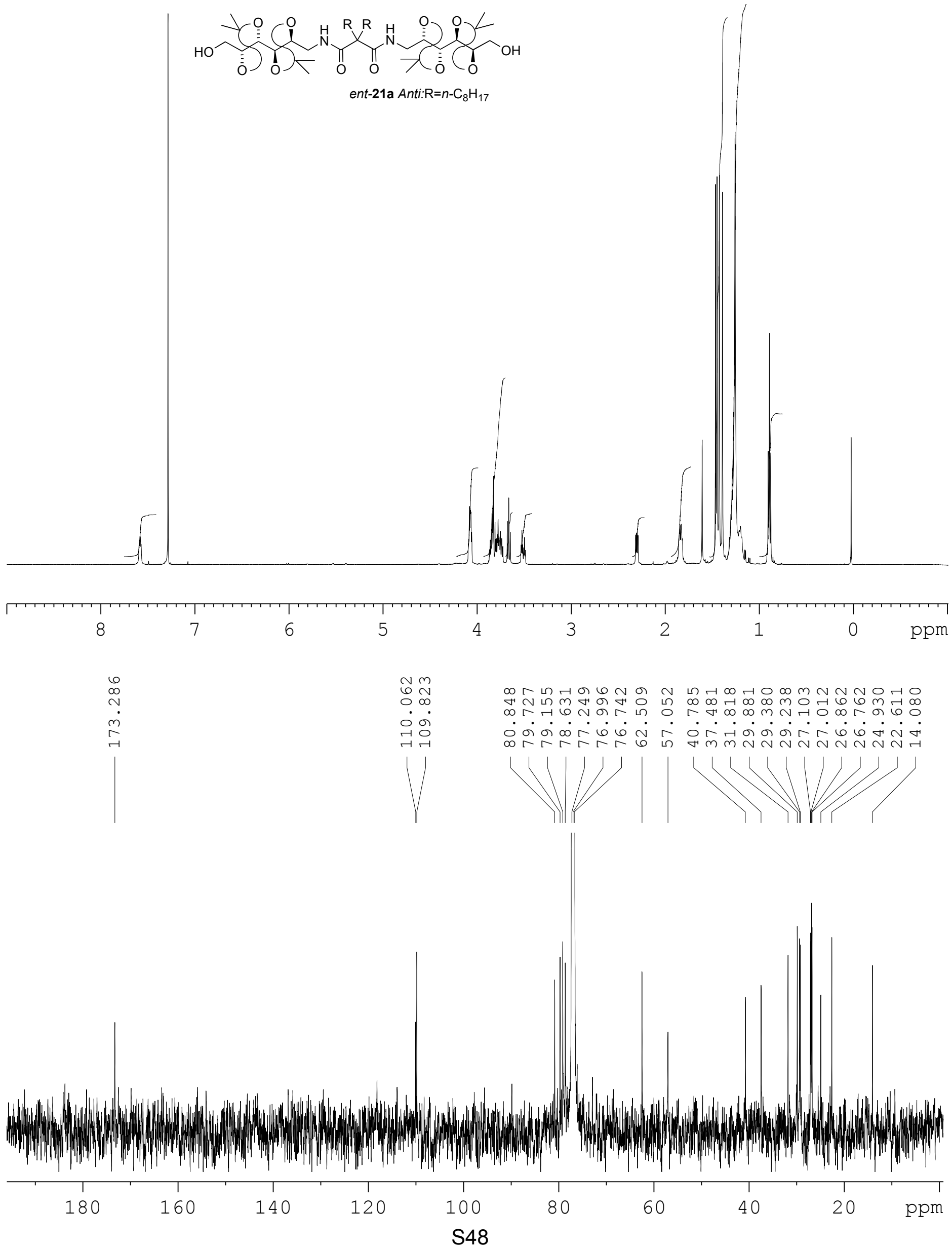

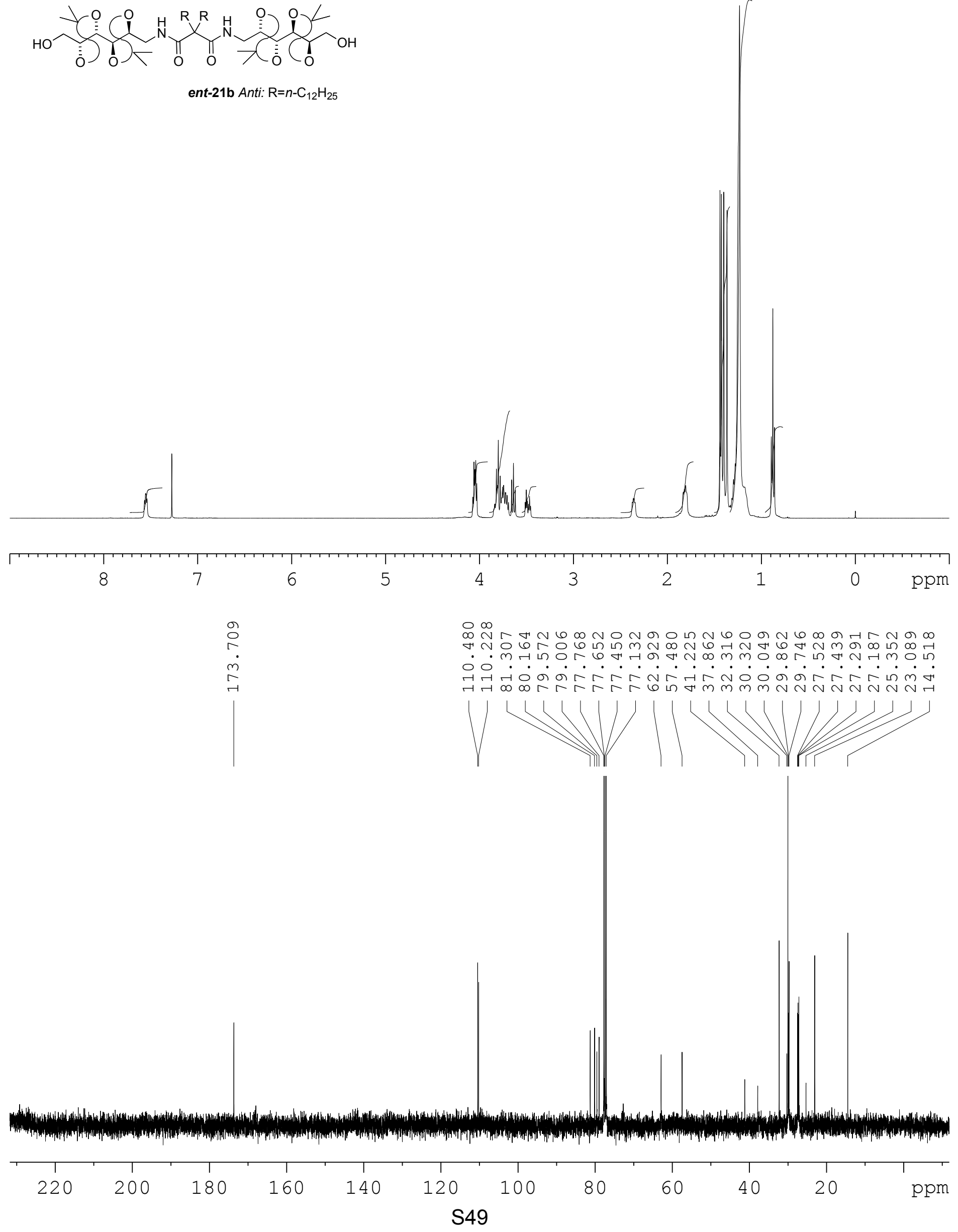
$\overbrace{\text { OMBO }}^{(O)_{\bar{O}}^{O}}$

22
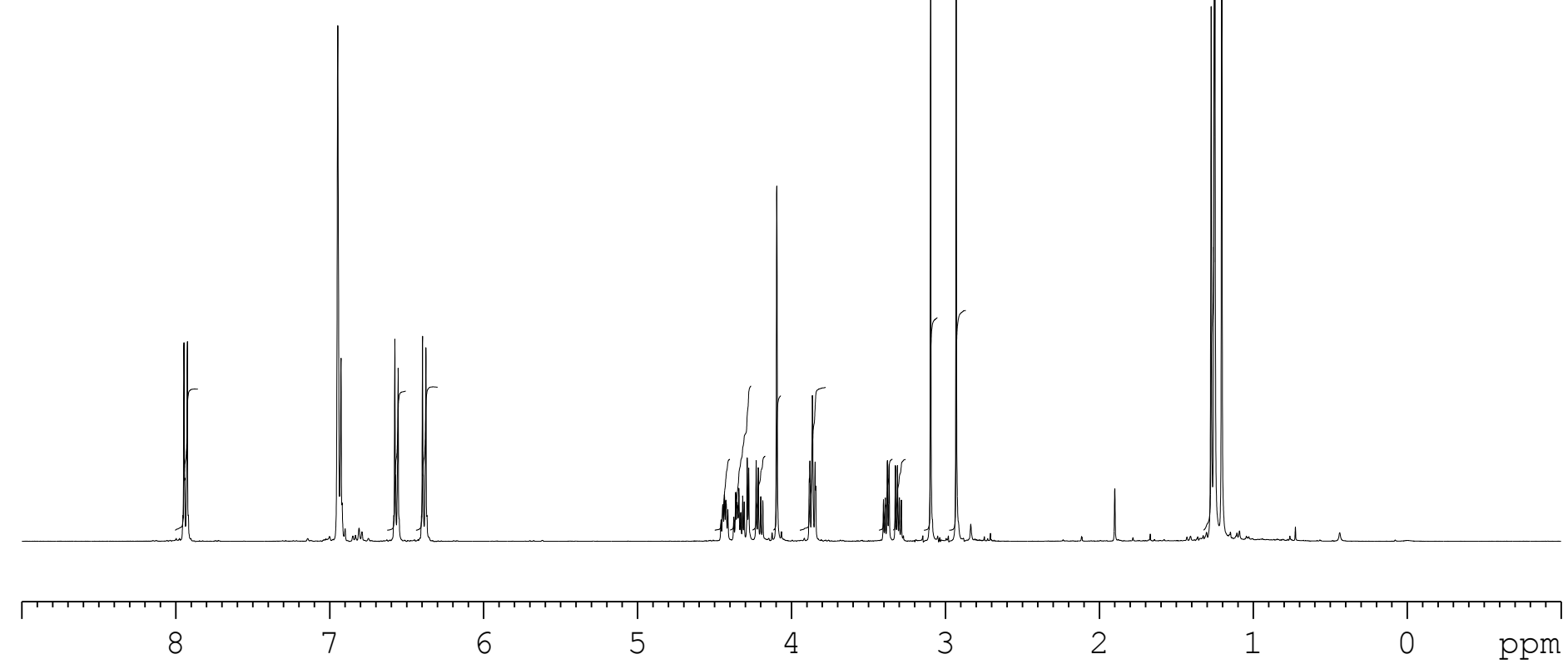

$\infty$ ㄴ HOOM⿻

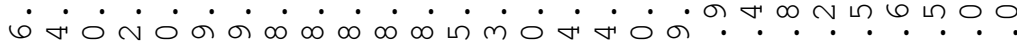

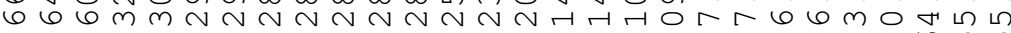

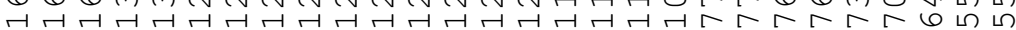
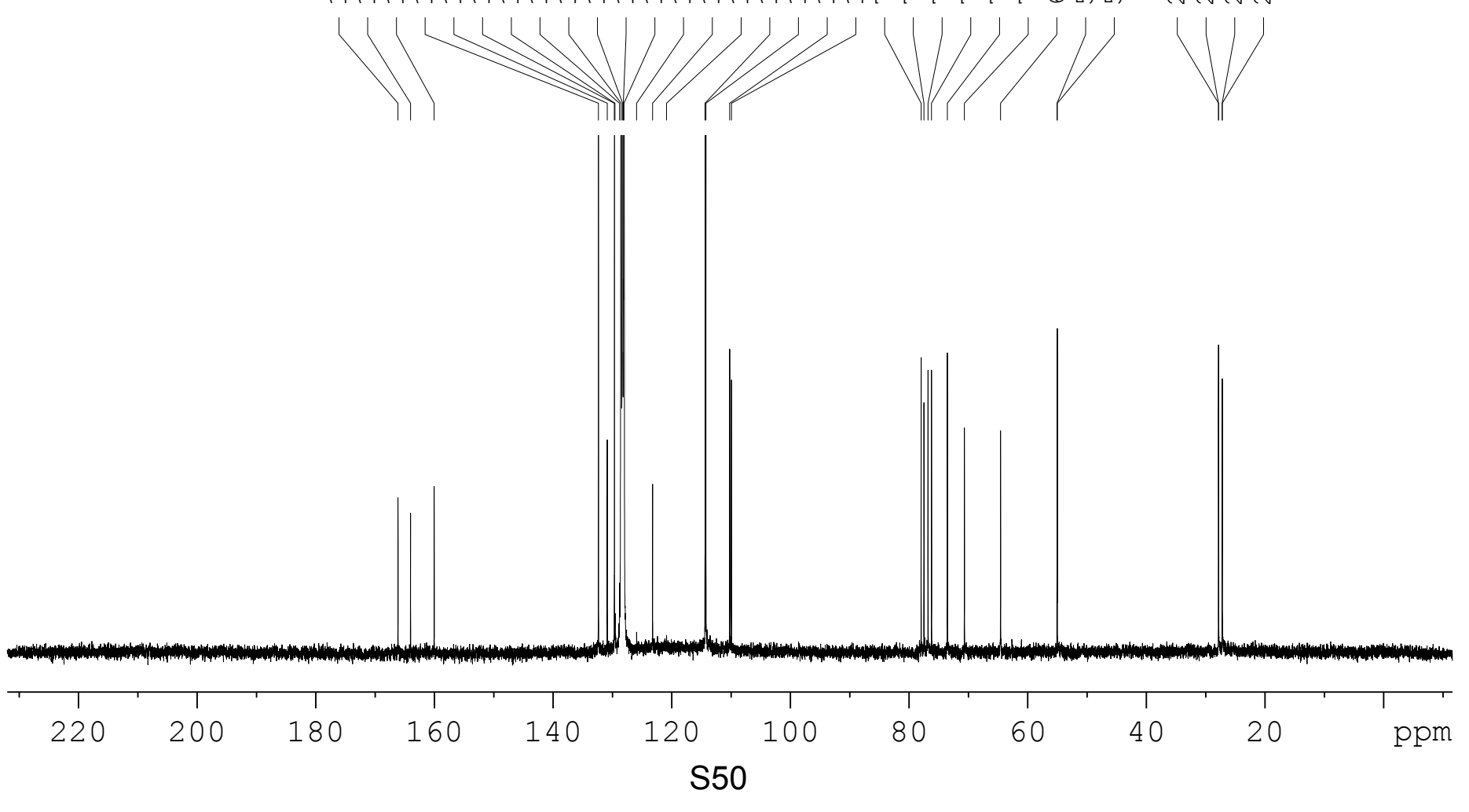

r $\forall m \sim n$ $\infty \infty \times$.

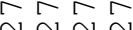

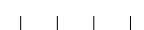




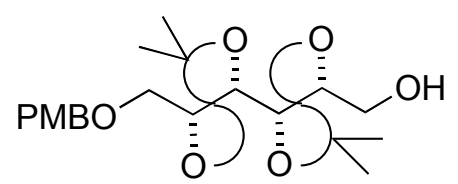

23
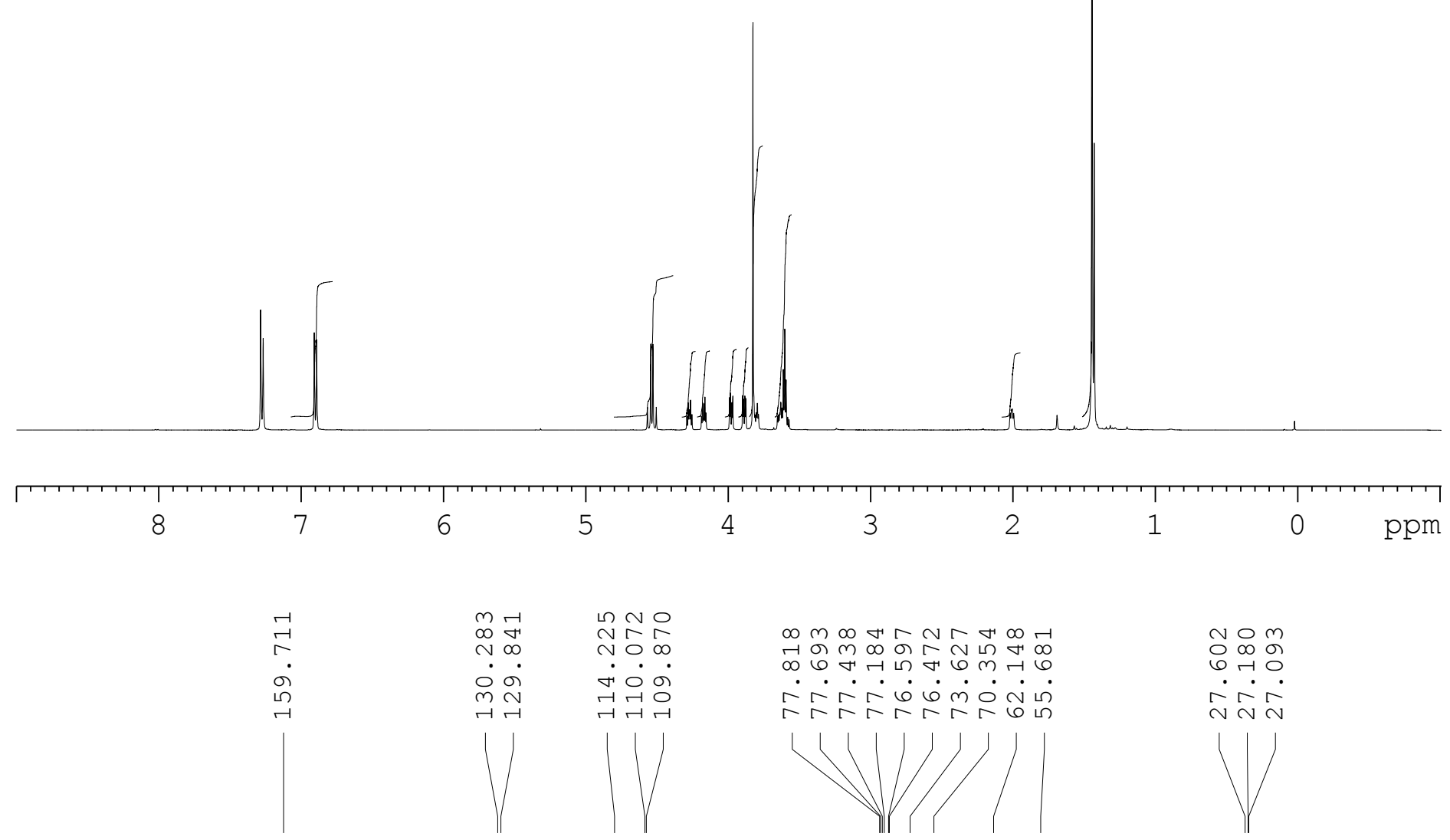

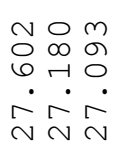

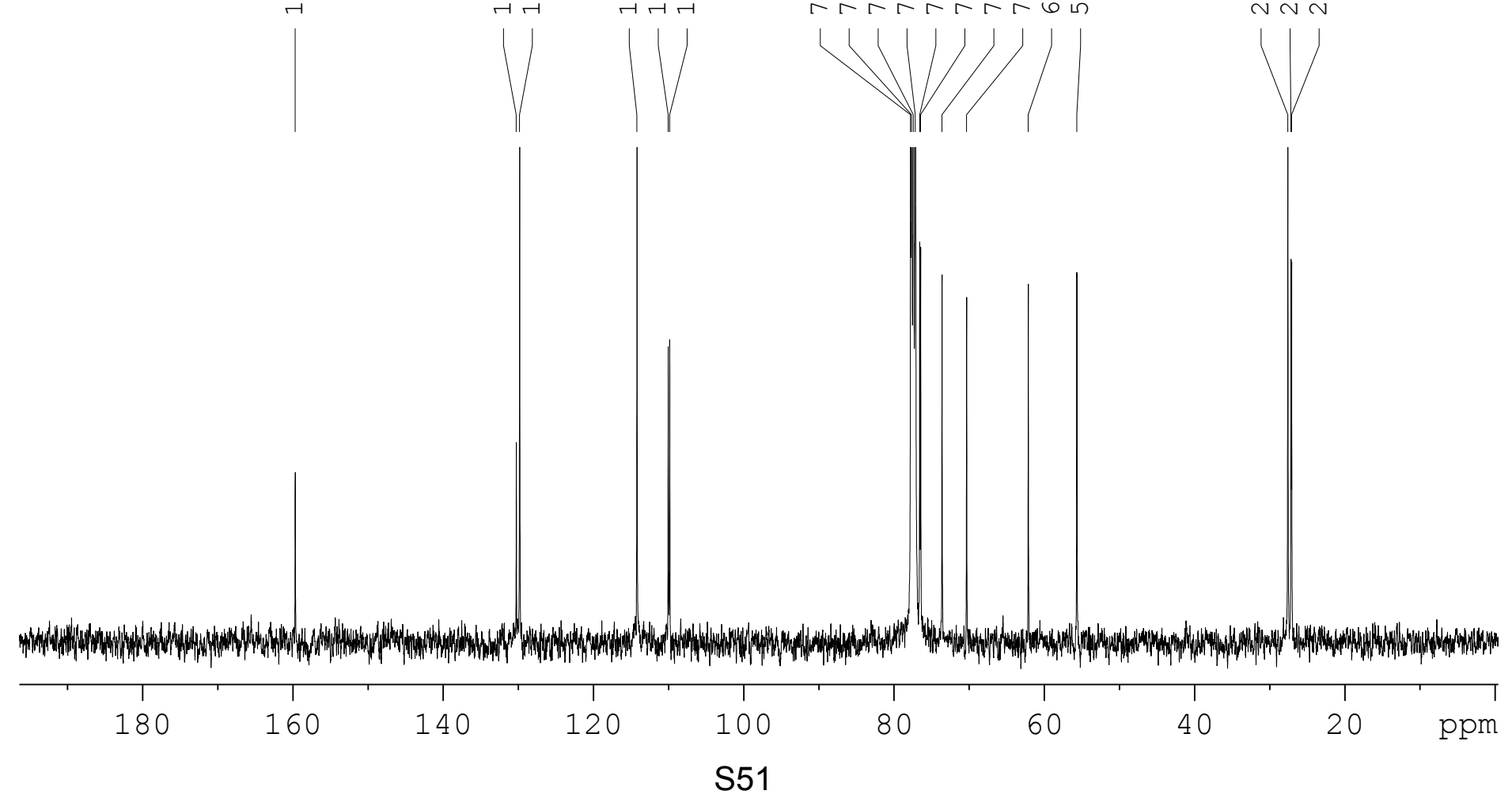



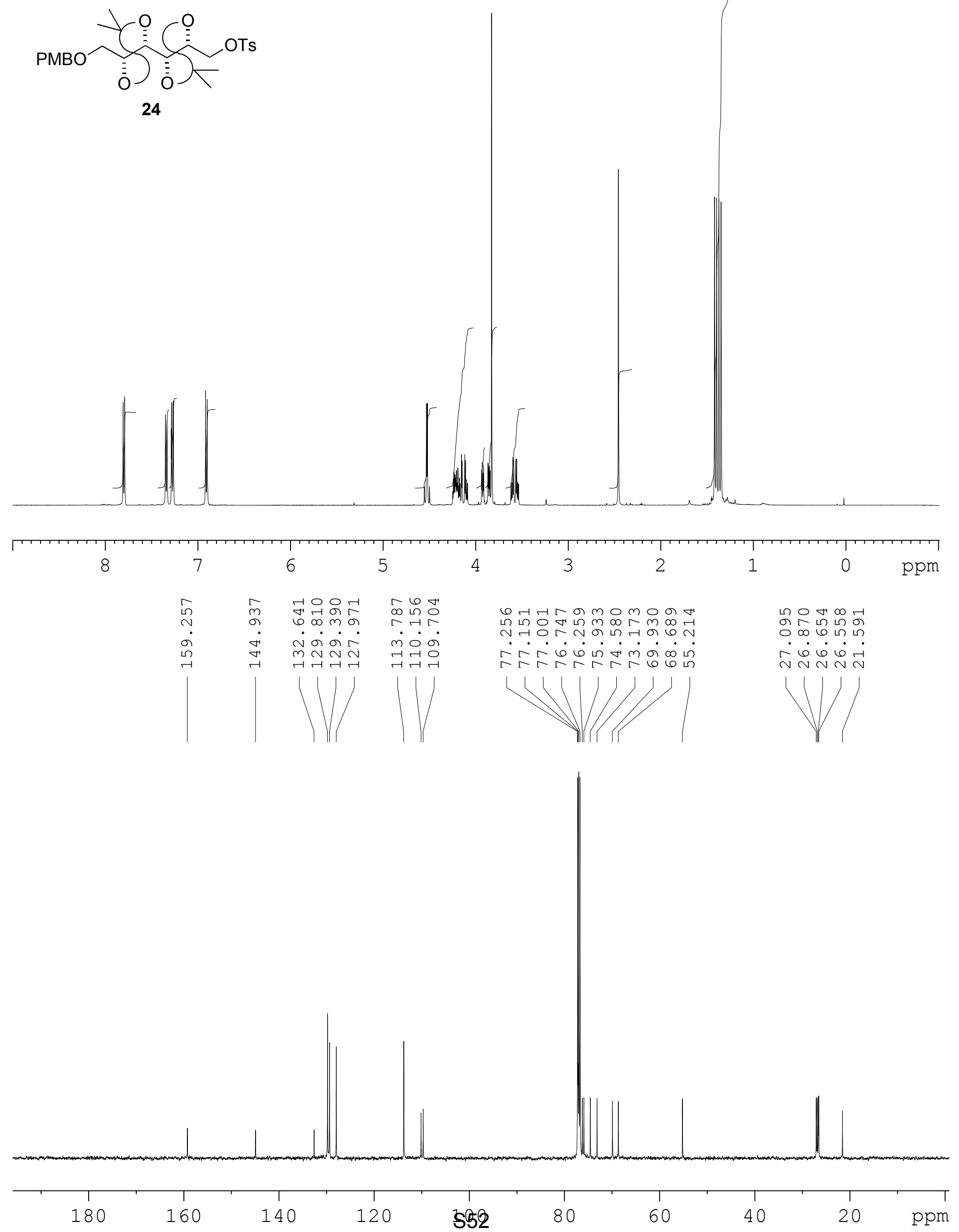


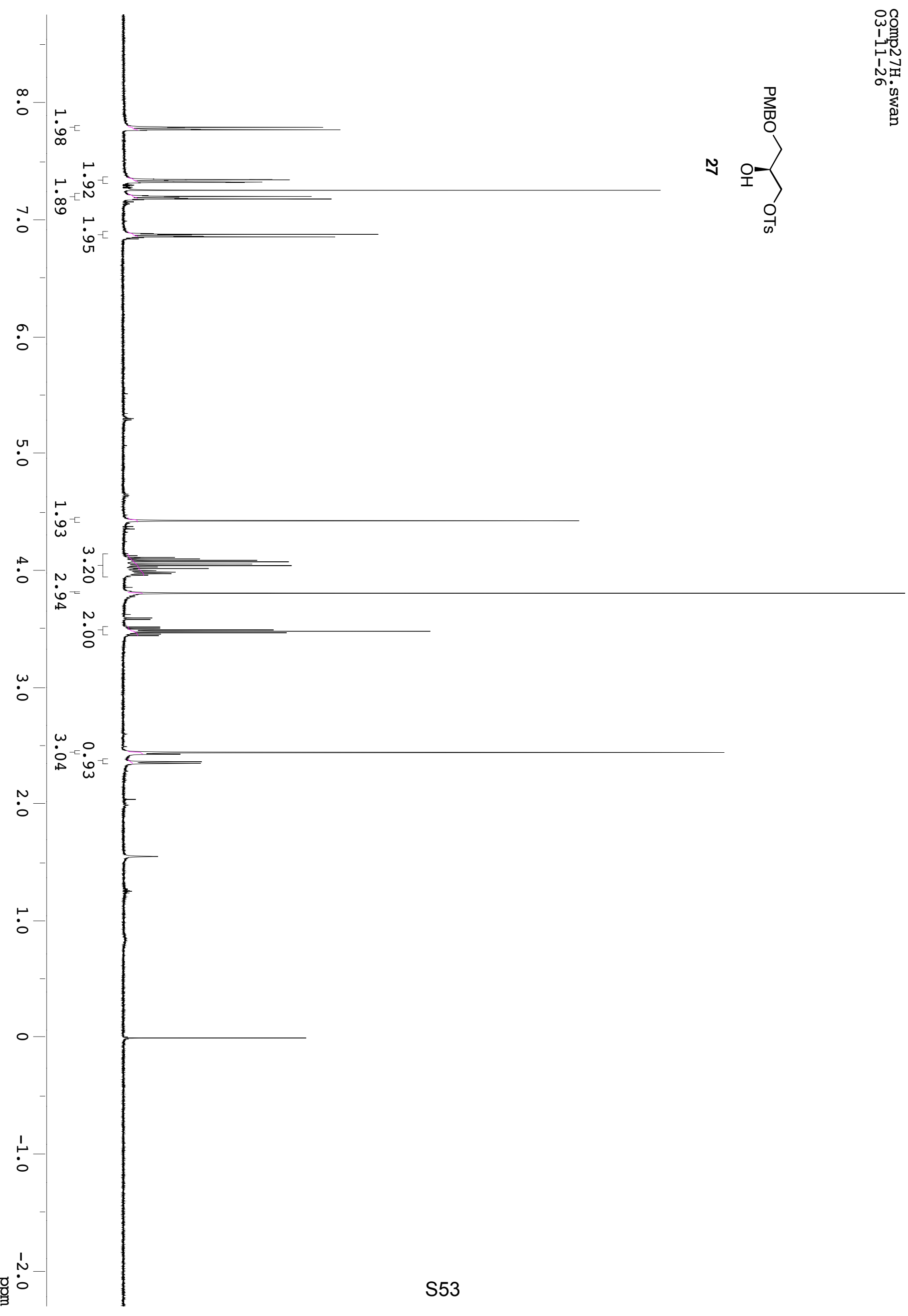




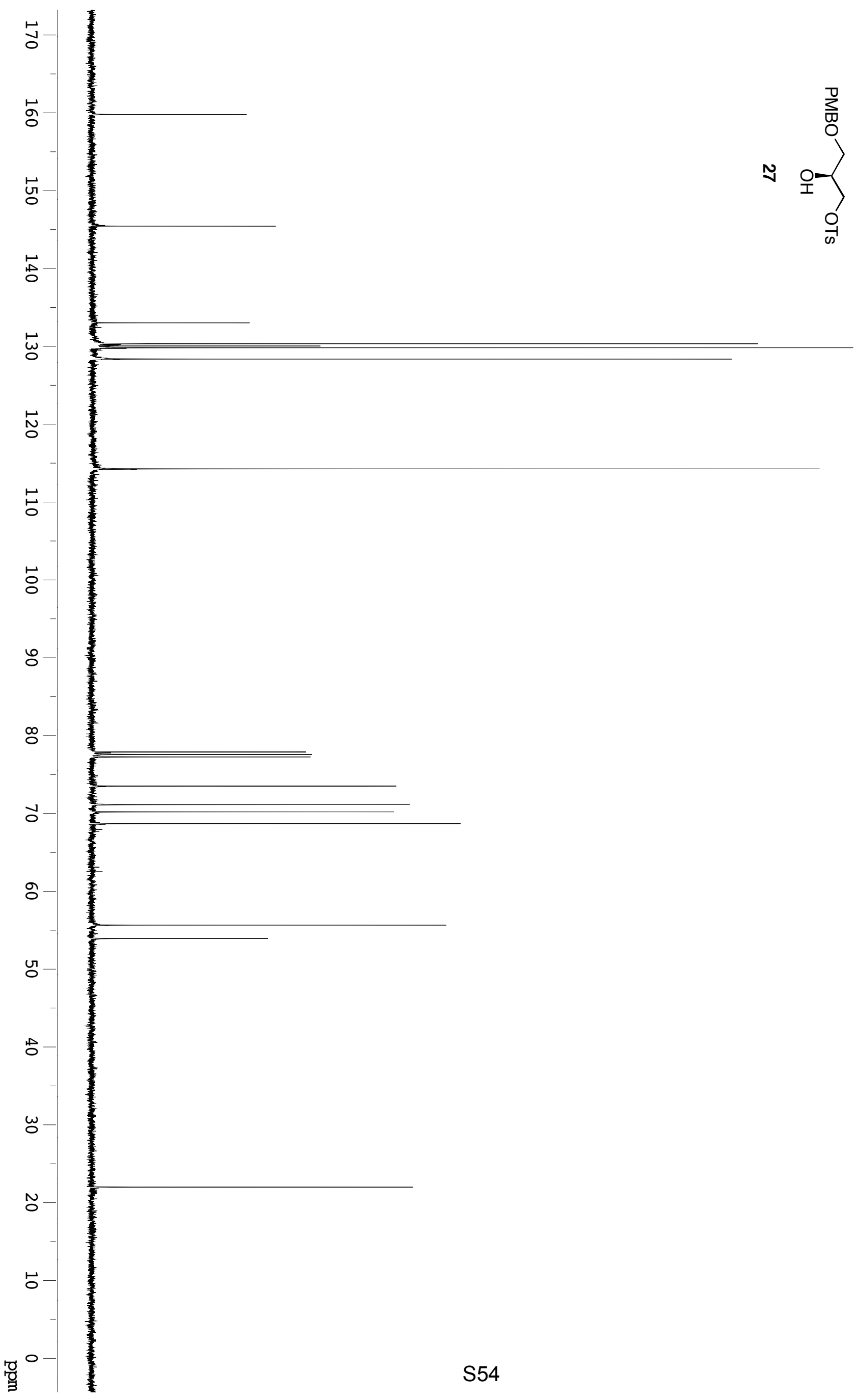


<smiles>[R]C([R])(C(=O)OC)C(=O)OC</smiles>

30b R=n- $\mathrm{C}_{12} \mathrm{H}_{25}$
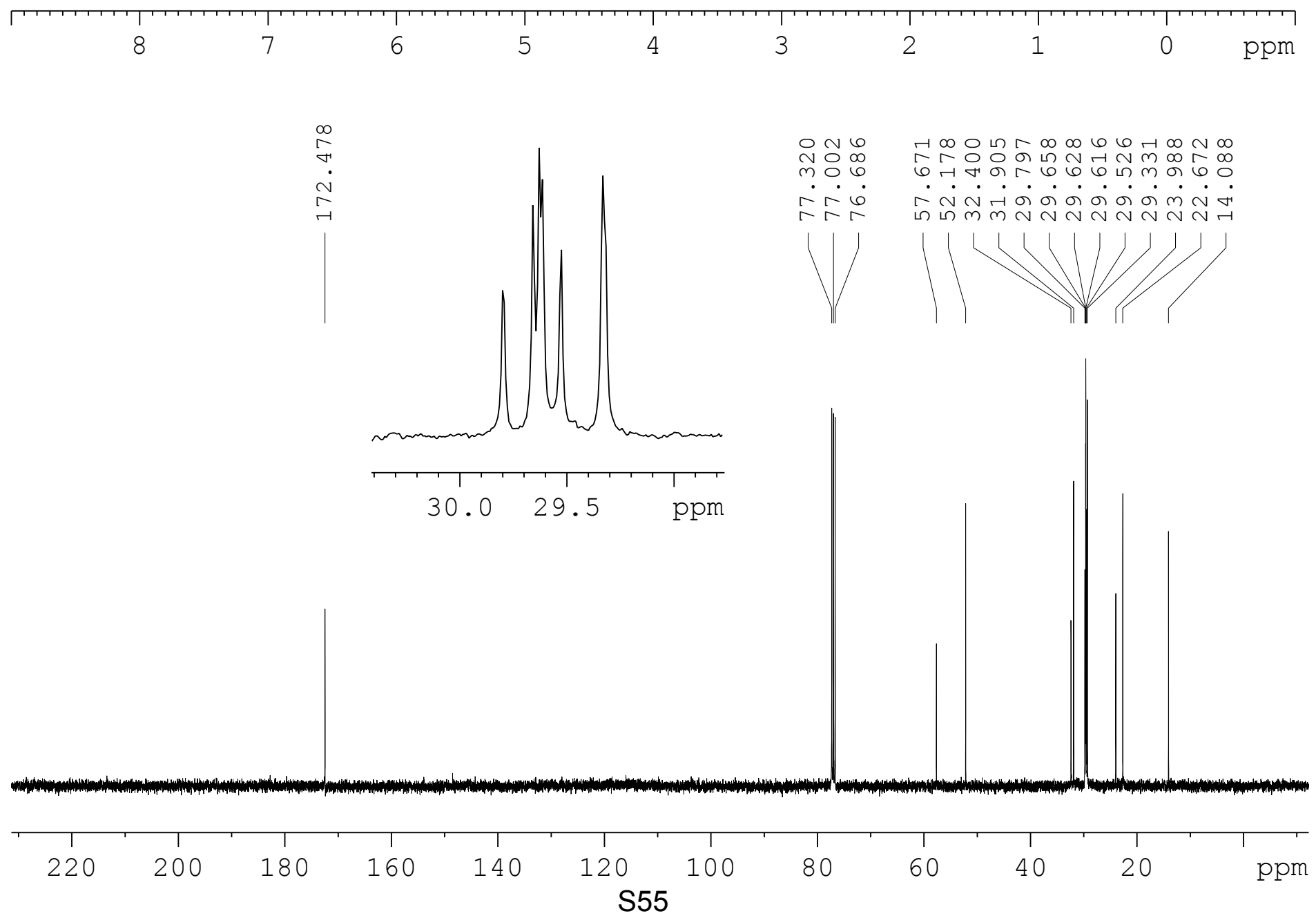


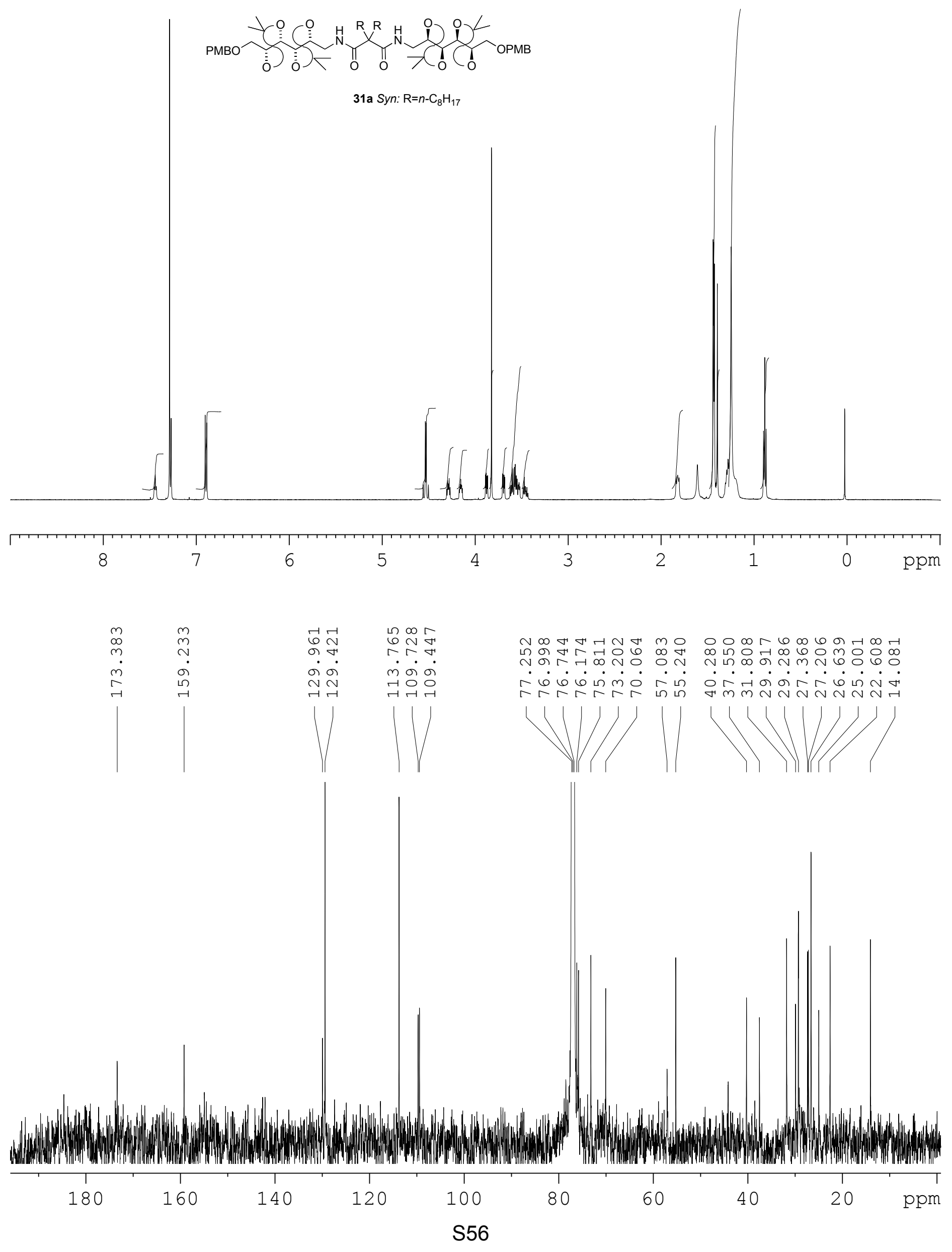




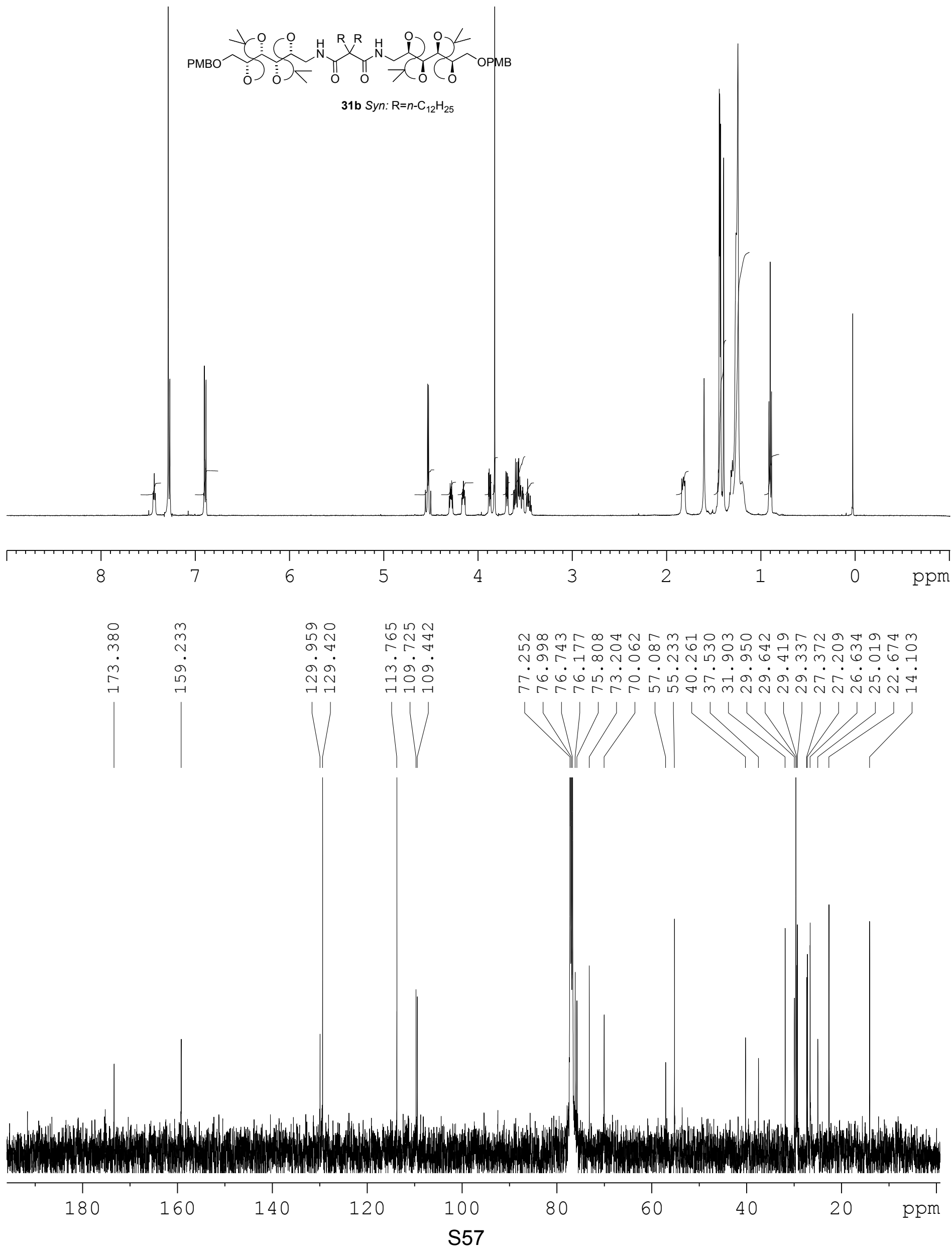




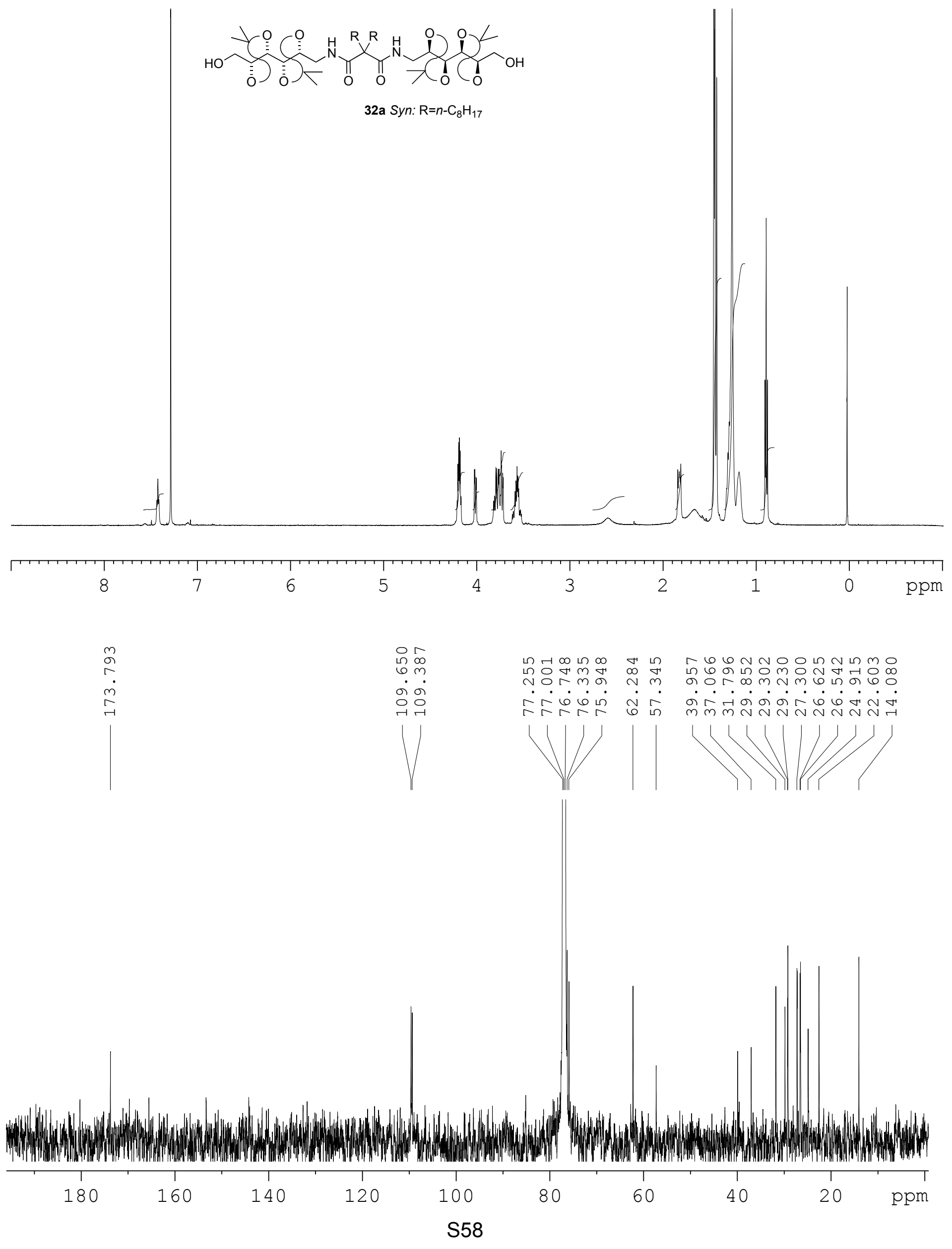



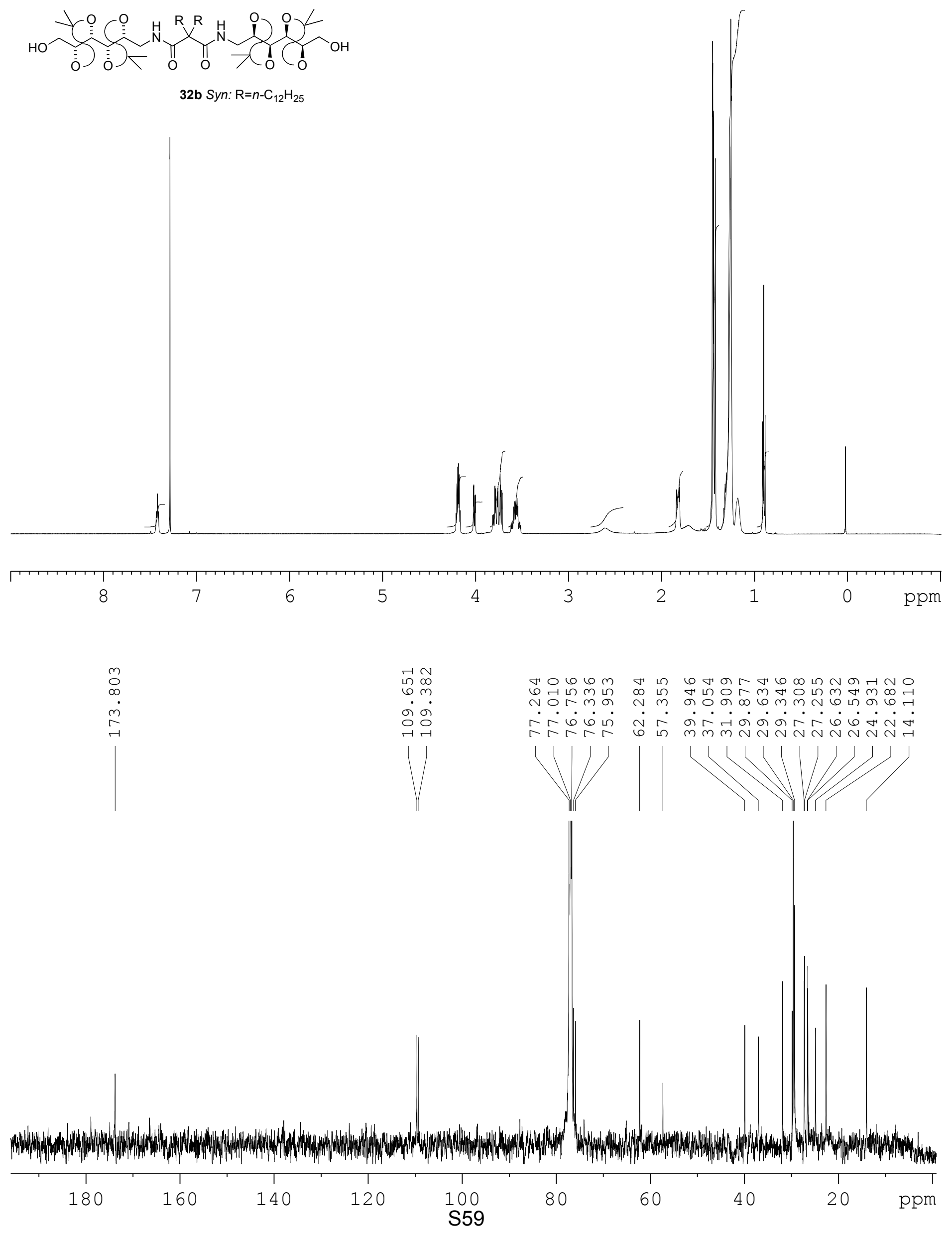